\title{
Cross Cultural Relationships of Depression, Attachment Styles, and Quality of Romantic Relationships: Cultural Difference between Taiwanese/Chinese and American College Students
}

\author{
Yi-An Lo Burleson \\ West Virginia University
}

Follow this and additional works at: https://researchrepository.wvu.edu/etd

\footnotetext{
Recommended Citation

Burleson, Yi-An Lo, "Cross Cultural Relationships of Depression, Attachment Styles, and Quality of Romantic Relationships: Cultural Difference between Taiwanese/Chinese and American College Students" (2013). Graduate Theses, Dissertations, and Problem Reports. 3634.

https://researchrepository.wvu.edu/etd/3634

This Dissertation is protected by copyright and/or related rights. It has been brought to you by the The Research Repository @ WVU with permission from the rights-holder(s). You are free to use this Dissertation in any way that is permitted by the copyright and related rights legislation that applies to your use. For other uses you must obtain permission from the rights-holder(s) directly, unless additional rights are indicated by a Creative Commons license in the record and/ or on the work itself. This Dissertation has been accepted for inclusion in WVU Graduate Theses, Dissertations, and Problem Reports collection by an authorized administrator of The Research Repository @ WVU. For more information, please contact researchrepository@mail.wvu.edu.
} 
Cross Cultural Relationships of Depression, Attachment Styles, and Quality of Romantic Relationships: Cultural Difference between Taiwanese/Chinese and American College Students

\section{Yi-An Lo Burleson}

Dissertation submitted to the College of Education and Human Services at West Virginia University in partial fulfillment of the requirements

for the degree of

Doctor of Philosophy

in

Counseling Psychology

Jeffrey Daniels, Ph.D., Chair

James Bartee, Ph.D.

Deborah Hendricks, Ed.D.

Daniel Hursh, Ph.D.

Monica Leppma, Ph.D.

Department of Counseling, Rehabilitation Counseling, and Counseling Psychology

\section{Morgantown, West Virginia \\ 2013}

Keywords: international students, attachment styles, depressive moods, romantic relationships, counseling 
Cross Cultural Relationships of Depression, Attachment Styles, and Quality of Romantic Relationships: Cultural Difference between Taiwanese/Chinese and American College Students

\author{
Yi-An Lo Burleson
}

\begin{abstract}
Relationship quality has been determined to be a positive factor in the treatment of depression (Brown, 2000; Fagan, 2009). Although the importance of marriage has been broadly studied, little research has investigated correlations among relationship quality, depressive moods, and attachment styles. Although the prevalence of depressive moods has been documented within populations of Taiwanese/Chinese international students and American college students (Wei et al., 2007), Wang and Mallinckrodt (2006) found that definitions of ideal attachment differ in these groups. Furthermore, researchers have not yet investigated the effect of cultural differences and attachment styles on the interactions between relationship quality and depressive moods among Taiwanese and Chinese international students.
\end{abstract}

Two-group and four-group comparison (Macready, 2005) methods were applied to answer the following questions: 1) Is there a significant difference between quality of romantic relationships or levels of depressive moods of American college students with anxious or avoidant attachment style and Taiwanese or Chinese college students studying in America with anxious or avoidant attachment style? 2) How does the effect differ between the two groups? A multivariate analysis of variance (MANOVA) was conducted to determine the effect on the linear combination of relationship quality and levels of depressive moods between the American students and Taiwanese/Chinese international students with different attachment categories (secure, preoccupied, dismissive, and fearful). Four follow-up analyses of variance (ANOVAs) were conducted to determine statistical significant differences in levels of depressive moods or relationship quality among Taiwanese/Chinese international students with different attachment categories. 


\section{DEDICATION}

I would like to dedicate this dissertation to many people in my life, especially my family. First, I want to express my deepest appreciation to my parents, 駱建樹 and 張有. I would like to thank my parents for giving me the freedom to study in the U.S. and providing support and continuous love during my graduate training. Throughout my life, you have taught me the significance of learning, diligence, and helping others. I am very grateful to be your daughter.

To my husband, John Burleson, thank you very much for your love, encouragement, acceptance, and care. You have almost always been the person who best understands, accepts, encourages, and cheers me on throughout my doctoral training, dissertation, internship, and job interviews. I am certainly blessed to have you in my life.

I would also like to thank my extended family in Taiwan and Maryland, my in-laws in North Carolina, and my church family in Morgantown. Because of your warmth, encouragement, and prayers, I have had the hope and strength to continue my learning and further exploration of academic, professional, and interpersonal areas both in Taiwan and America. I have great memories of all of you, and your words have motivated me to continue developing my knowledge and skills.

To my dear friends Li-Yu Chen, Chih-Ning Chang, and Chu-Yu Chang, thank all of you so much for your emotional support. Your enthusiasm and dedication to children, adolescents, and families has inspired me to further strengthen my courage, perseverance, and patience to serve people and engage in research. I am truly grateful to have both of you as life-long friends. 


\section{ACKNOWLEDGEMENTS}

I would like to acknowledge the support and guidance I have received from a number of professors throughout my graduate work in general as well as during this dissertation process in particular. I am really grateful to have received support, comments, critique, and guidance from my dissertation committee members throughout this process. Thank you for dedicating your time and effort to my dissertation work.

I would especially like to express appreciation to my dissertation chair, Dr. Jeffrey Daniels, for your support, time, and effort. I have learned a great deal from you about writing literature reviews, methodology development, and conducting dissertation research. I would also like to thank Dr. James Bartee for providing support, consultation, and training me to be a more advanced, flexible, and self-aware clinician. You have shown me the significant impact of positive regard, dedication, and genuineness. I would like to thank Dr. D. J. Hendricks for your tremendous assistance and guidance on the statistical analyses for this project. With your assistance, I was able to extend my understanding of more statistical analyses and interpretation of the results. I would also like to express my gratitude to Dr. Daniel Hursh, for your feedback, support, and encouragement in the later development of this dissertation. I am so blessed to have had you on my committee. I would also like to acknowledge Dr. Monica Leppma, for providing detailed feedback to my writing and helpful comments to the study. Thank you for your time and effort in the process as well as to the many other professors not mentioned here.

I would also like to acknowledge the contribution and express my appreciation to the faculty and staff in the Department of Counseling, Rehabilitation Counseling, and Counseling Psychology. Special thanks to Vicki Railing, who has been exceptionally helpful in assisting me with administrative issues throughout the completion process of my dissertation. I appreciate the teaching, training, and guidance from every faculty member in the Counseling Psychology program at West Virginia University, who has trained me well, challenged me, and encouraged me to strengthen my skills and integrate my cultural background into my professional and personal journey as a psychologist-in-training.

And last, but certainly not least, many thanks go out to Margalit Persing, who edited this dissertation.

I will take the lessons I have learned from all of you with me as I start my professional life. 


\section{TABLE OF CONTENTS}

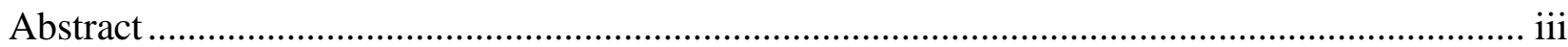

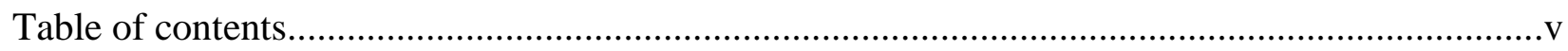

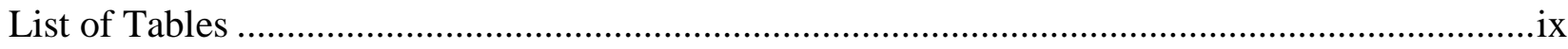

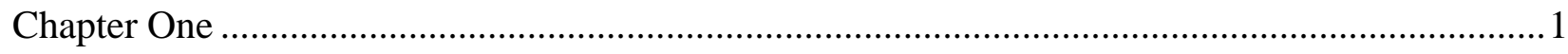

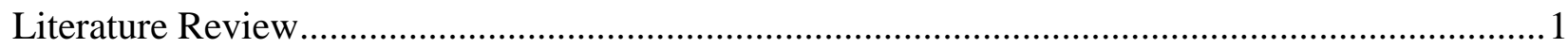

Theoretical Bases of Romantic Relationships ...................................................................4

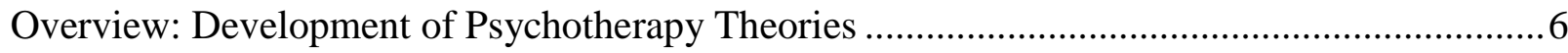

Cultural Differences and Impact of Acculturation........................................................

Romantic relationships as social support for Asians...............................

Cultural differences in interpersonal relationships................................ 13

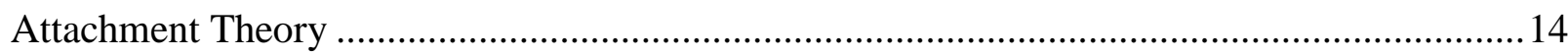

Theoretical Concepts and Early Studies concerning Attachment Styles ............................... 16

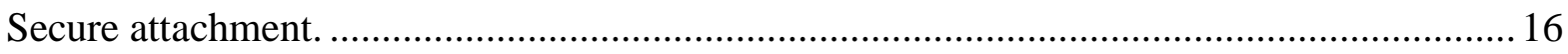

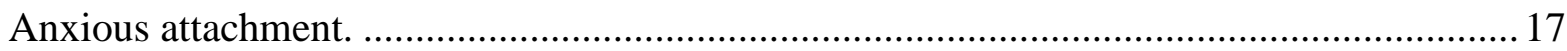

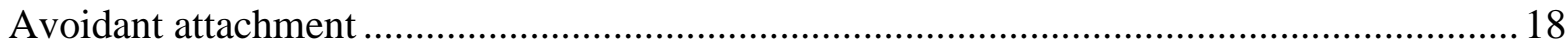

Inconsistent Findings about Adult Romantic Relationships ...........................................20

Interactions among Attachment Styles, Depressive Moods, and Cultural Differences .............22

Attachment styles and depressive moods......................................22

Depressive moods and attachment styles vs. individualistic and collectivistic cultures......25

Impact of positive and negative relationships and correlations to depressive moods .............. 31

Good relationship as a mediator of depressive moods................................................. 31

Marital conflict and depressive moods........................................... 34 


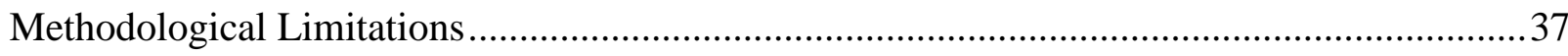

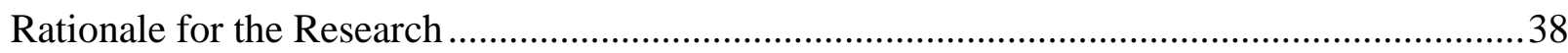

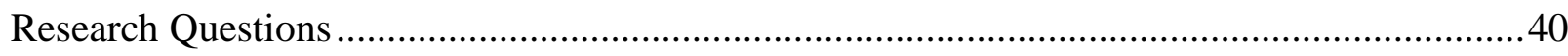

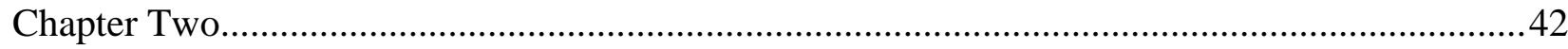

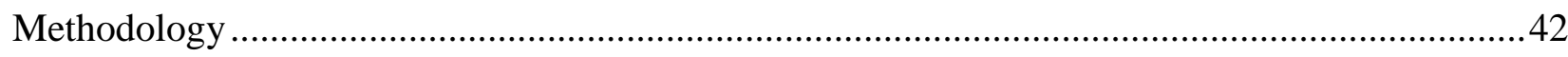

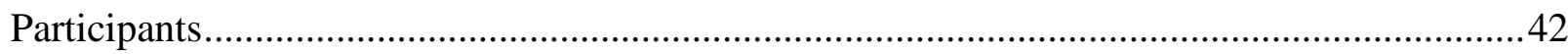

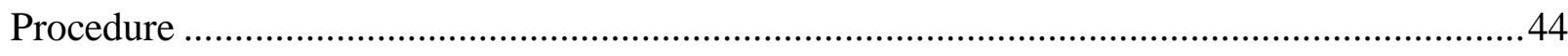

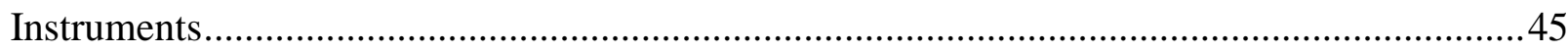

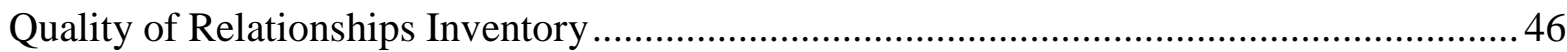

Experiences in Close Relationship Scale-Short Form..................................47

Center for Epidemiologic Studies Depression Scale ............................................................. 48

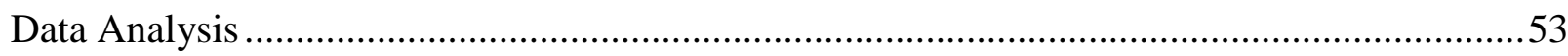

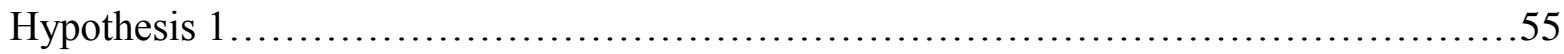

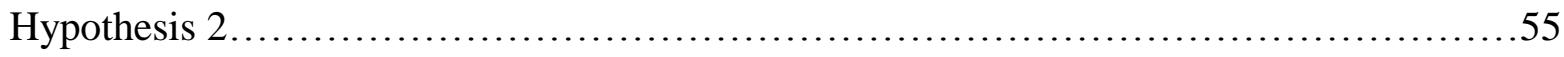

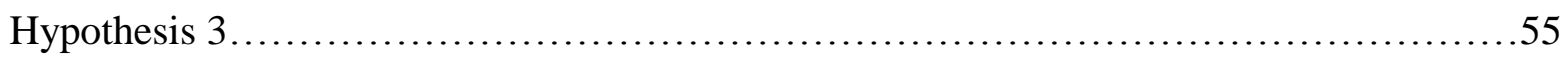

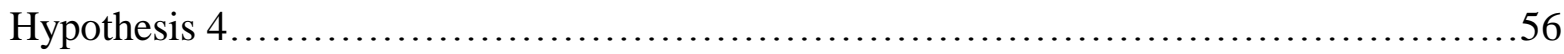

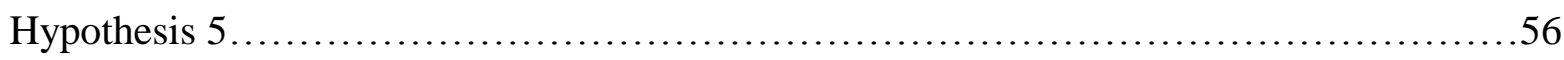

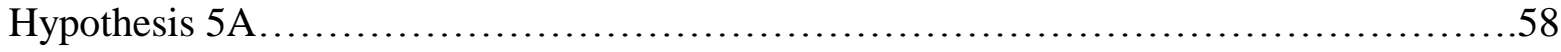

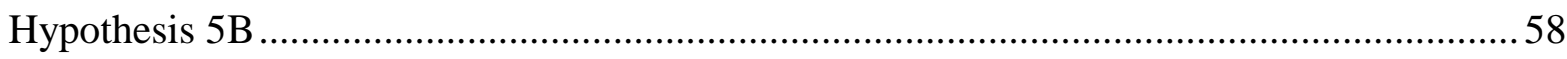

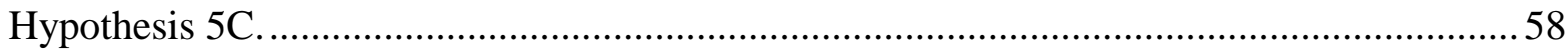

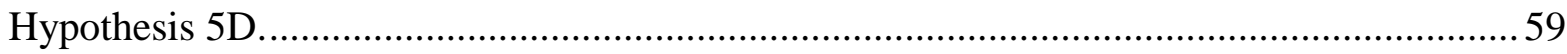

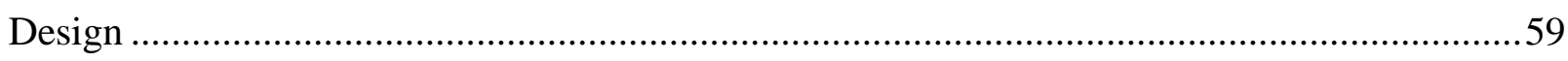

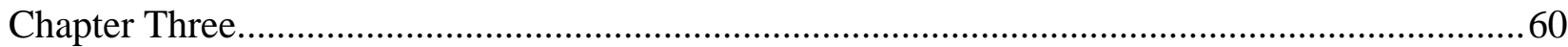




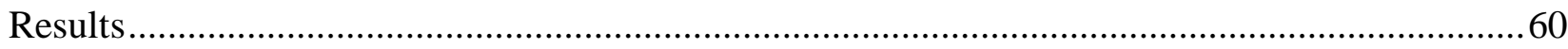

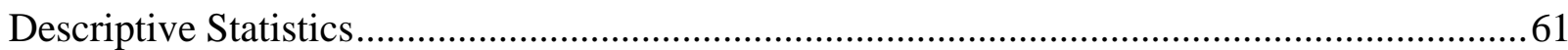

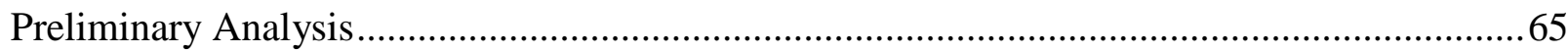

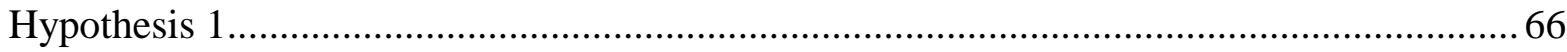

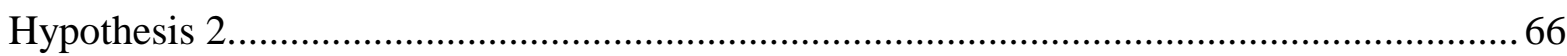

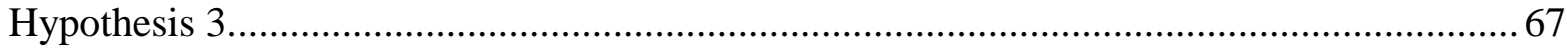

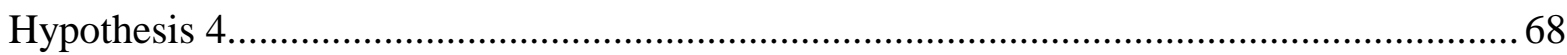

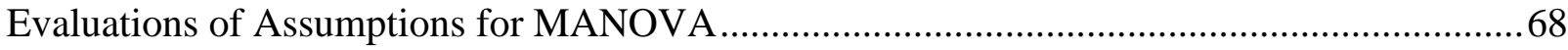

Tests of representativeness, sample size, normality, and levels of measurement.................. 68

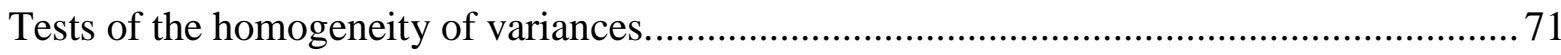

Linearity relationship between dependent variables..................................................... 71

Homogeneity of variance-covariance matrices.......................................................... 71

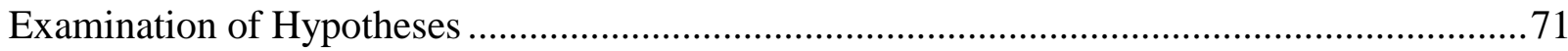

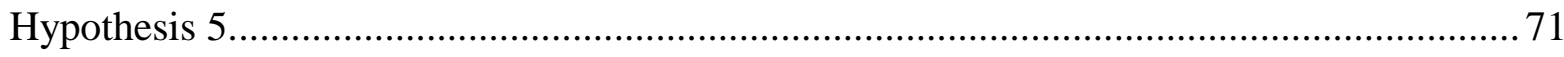

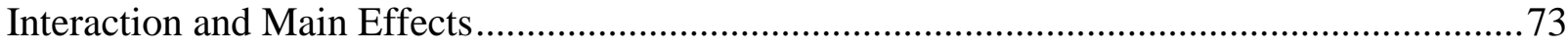

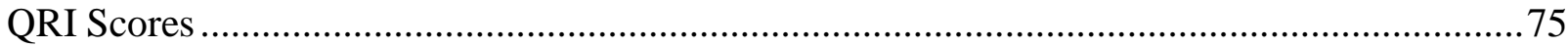

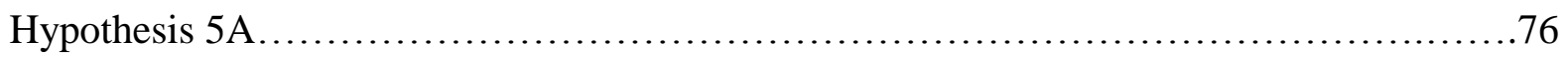

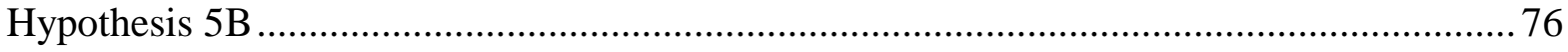

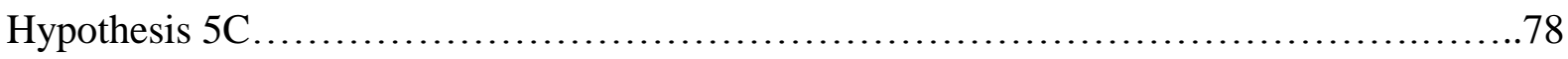

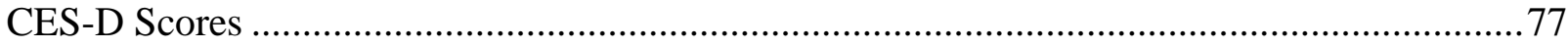

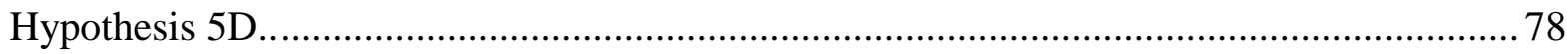

Qualitative Analysis: Factors Impacting Romantic Relationships ......................................79

Cultural Effects and Differences in Romantic Relationships .......................................... 80 


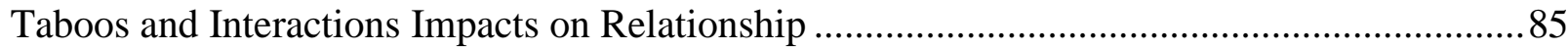

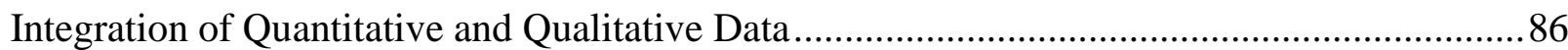

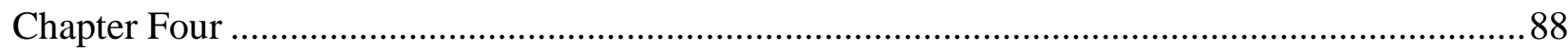

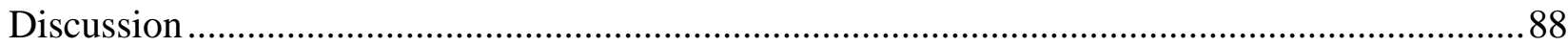

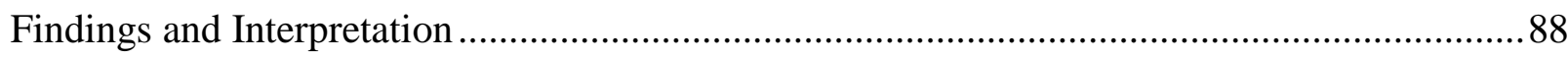

Theoretical and Clinical Implications of the Findings ........................................................ 95

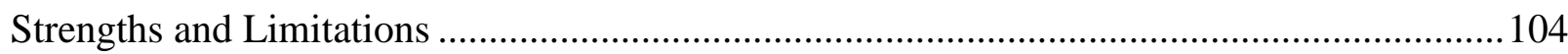

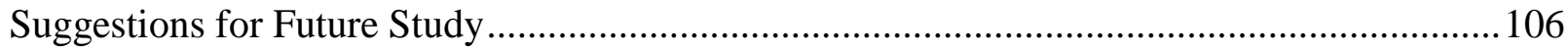

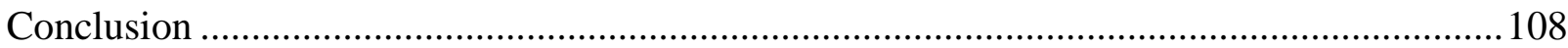

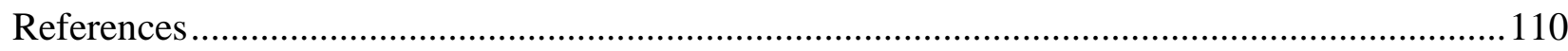

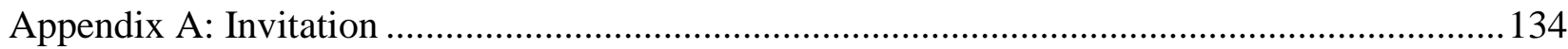

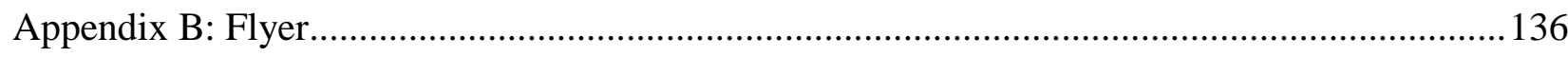

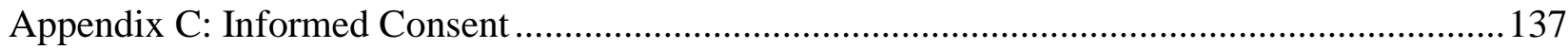

Institutional Review Board ........................................................................................ 137

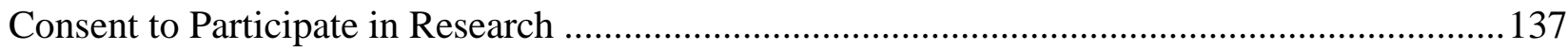

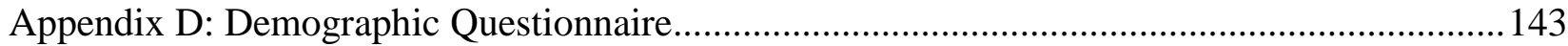

Appendix E: Quality of Relationships Inventory....................................................... 145

Appendix F: Experiences in Close Relationship Scale-Short Form ..................................... 148

Appendix G: Center for Epidemiologic Studies Depression Scale...........................148 


\section{LIST OF TABLES}

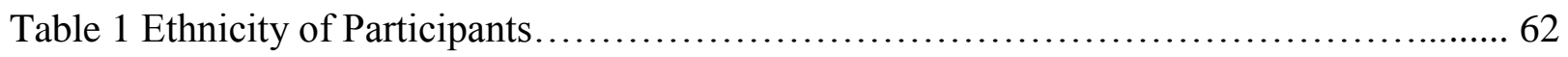

Table 2 Number of Children in Residence.......................................... 63

Table 3 Employment....................................................... 63

Table 4 Means, Standard Deviations, and Percentage of Anxious and Avoidant Participants

in Chinese/Taiwanese International Students and American Students.......................64

Table 5 Means, Standard Deviations of Depressive Moods, Relationship Quality of

Participants with High Attachment Anxiety........................................ 65

Table 6 Means, Standard Deviations of Depressive Moods, Relationship Quality of

Participants with High Attachment Avoidance.......................................65

Table 7 Skewness and Kurtosis of Dependent Variables............................. 69

Table 8 MANOVA on Relationship Quality and Depressive moods between American and

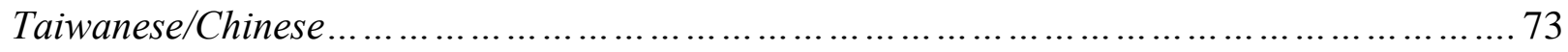

Table 9 ANOVA on Relationship Quality and Depressive moods between American and

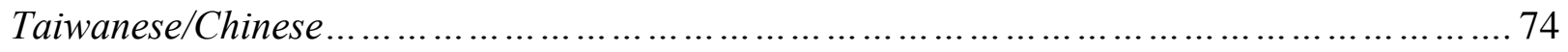

Table 10 Relationship Quality, Four Attachment Style, and Ethnicity or Home Country....... 75

Table 11 Depressive Moods, Four Attachment Style, and Ethnicity or Home Country........... 77 


\section{CHAPTER ONE}

\section{LITERATURE REVIEW}

Despite the current trend toward multiculturalism, psychologists have struggled to enhance multicultural competence due to limited research conducted within specific cultural minority groups. General guidelines toward improving therapist awareness, knowledge of a specific cultural group, and skills have been highlighted in training for multicultural competence (American Psychological Association, 2002; Sue \& Sue, 2007). However, few specific therapeutic approaches have been recommended for adequately serving the various ethno-centric needs of international students or discrete ethnic minority groups, such as Asian Americans, Hispanic Americans, and African Americans. The specific therapeutic procedure and approaches for effective treatment based on a theoretical orientation and intra-ethnic differences (e.g., differences among Korean, Chinese, and Japanese within the "Asian" ethnic group) have not yet been fully explored (Chu, 2007; Sue \& Zane, 2009).

While research on specific therapeutic approaches for cultural minorities is limited, the increasing numbers of culturally distinct Asian international students in the United States expands the need for studies targeting this population (Wei et al., 2007). According to the Chronicle of Higher Education (2009) and Defoe (2009), Asian international students comprised $62 \%$ of all international students. While Indian students were the biggest international student population, Chinese international students were the second biggest population among international students. Students from China represented the fastest growing international population from 2007 to 2009 . Multiple researchers have documented significant cultural differences between Americans and Asian/Asian Americans in values (Kim, 2010; Kim, Atkinson, \& Yang, 1999; Raghavan, 2006; Sue \& Sue, 2003, 2007), social systems (collectivistic 
and individualistic; Kuo, 2004), communication styles (Park \& Kim, 2008), self-efficacy, emotion regulation (Cheung \& Park, 2010), emotional reactivity while depressed (ChentsovaDutton, Tsai, \& Gotlib, 2010), help-seeking experience (Kim, 1996), and emotion expression (Kitayama, Mesquita, \& Karasawa, 2006).

In addition to different cultural values, Asian international students have the disadvantage of appearing "foreign," unlike European international students, who share many physical characteristics with Caucasian Americans who are the majority in the United States. Thus, Asian international students frequently experience similar microaggressions as Asian Americans (Sue, Bucceri, Lin, Nadal, \& Torino, 2009). Because Asian international students experience more discrimination or microaggression than do European international students (Sodowsky \& Plake, 1992), the negative experience may further lead to depression. Other research has demonstrated that perceived racism may be significantly associated with increased levels of depression (Priest, Paradies, Stewart, \& Luke, 2011). Consistent with the multiple barriers and high likelihood of experiences of racism for Asian international students, approximately $30 \%$ of the Asian international students reported significantly high levels of depressive symptoms based on a selfreport measure, Center for Epidemiologic Studies Depression Scale (CES-D; Wei et al., 2007). The high prevalence and significance of depression or depressive moods as well as the barriers to mental health services for Asian internationals should not be overlooked by mental health professionals and educators.

International students tend to experience depressed moods or depression during the process of adjustment, resulting from acculturation stress and lack of social support (Adler, 1975; Furukawa, 1997; Leong \& Chou, 1994; Leong \& Sedlacek, 1986). Among the international students who attend therapy sessions in college counseling centers, depression is 
one of the most common presenting problems (Yi, Lin, \& Kishimoto, 2003). Depression and depressive moods are prevalent among Asian international students because, while experiencing transitional concerns in adjusting to college life similar to those of White American college students, the Asian students also face a number of additional obstacles (Leese, 2010). First, Asian international students share the minority status with other ethnic minorities in the United States and, as such, unlike their White American counterparts, they are more prone to experience psychological distress from racial discrimination or microaggression (e.g., Constantine, Kindaichi, Okazaki, Gainor, \& Baden, 2005; Lipsicas \& Mäkinen, 2010). Furthermore, Asian international students often face substantial language barriers and other forms of culture shock upon arriving in the United States. America's individualistic culture is significantly different from the collectivistic culture shared by most Asian nationalities (Choi, 2006; Sato \& Hodge, 2009; Tochkov, Levine, \& Sanaka, 2010). These stressors also give rise to adjustment issues not inherent in the collegiate transition experience of White American college-goers. More specifically, Tochkov, Levine, and Sanaka (2010) stressed that international students frequently face unique difficulty due to uncertainty of role expectations, language barriers, loss of environmental familiarity, and challenges in reestablishing social support. The relevance of these impediments to Asian International Students was also confirmed by Sato and Hodge's empirical findings.

In general, depression can cause serious impairment in individuals and often results in poor quality of life or even suicide (Pincus \& Pettit, 2001). Pincus and Pettit stated that if the severity of depressive symptoms cannot be reduced, many depressed individuals may end their lives by committing suicide. Because suicide exacts such a high price on society, it is important to determine how natural supports, such as romantic relationships, may relate to any decrease in 
the level of depressive symptoms in individuals. In the same vein, depressive moods in the normal population were found to significantly influence effectiveness in social, marital, and vocational functioning (Abakoumkin, Stroebe, \& Stroebe, 2010; Rozzini, 1996; Wang et al., 2007). The detrimental outcome of severe depression and the relationships between depression/depressive moods and quality of interpersonal relationships among reviewed studies further leads to the need for research on the effect of these two factors for Asian international students. Despite the general negative effect of depression/depressive moods on interpersonal relationship and social support (e.g., Mak, et al., 2010; Ritakallio, Luukkaala, Marttunen, Pelkonen, \& Kaltiala-Heino, 2010), little research has emphasized the relationship of quality of dating relationships on depression or depressive moods. Thus, this study focused on the interplay of quality of romantic relationships and depressive moods.

\section{Theoretical Bases of Romantic Relationships}

Attachment theory as put forth by Bowlby and Ainsworth (1991) and the triangular theory of love by Sternberg (1986) are the two most broadly cited theories concerning romantic relationships (Wang, 2006). Although Sternberg's triangular model provides an analysis of the three components of love, including intimacy, commitment, and passion, due to the relational nature of attachment theory (e.g., partner interaction and expectations) and the highly valued interpersonal relationships in the targeted population of the Taiwanese and Chinese international students, this study will further investigate the target population through attachment theory. Furthermore, there is little research applied to determine the relationship between depressive moods and the type of love in Sternberg's theory. Consistent with Mahler, Pine and Bergman's (1973) observation about the significant impact of early separation between infants and their mothers, Bowlby and Ainsworth postulated three major attachment styles: secure, anxious (or 
anxious-ambivalent by Hazen \& Shaver, 1994), and avoidant as developed from parent-child interactions during early childhood and infancy. Recent researchers established evidence of the impact of attachment styles on adolescent and adult romantic relationships (e.g., Cramer, 2004; Welsh, Grello, \& Harper, 2003).

Although recognized, the effects of levels of depressive moods related to the quality of romantic relationships have not been thoroughly studied. A review of extant literature about the relationship between romantic relationships and depressive moods reveals that most research to date has focused on the impact of conflictual marriage (Barnes, 2006), the poor parent-child relationship (Branje et al., 2010; Milan, Snow, \& Belay, 2009), or anxious attachment (Lee \& Hankin, 2009) on depressive moods. With closer connection or correlation to this dissertation study, Cramer (2004) studied adult relationship satisfaction, depressive moods, and attachment styles (Cramer, 2004). Although the impact of insecure attachment and depressive moods on relationship quality is well established for Caucasian American college students, research has been applied to the majority of Caucasian American college students but has not focused on the applicability of this theory to ethnic minority and international students in the United States (Wang \& Ratanasiripong, 2010; Wang \& Scalise, 2010).

Although the attachment theory has been broadly studied within the American population, particularly in Caucasian American college students (e.g., Williams \& Risking, 2004), research has shown inconsistent findings regarding the effect of anxious attachment styles on depression, or the impact of attachment anxiety on the quality of romantic relationships for the American population (e.g.,, Harwood, 2008; Shaver, Schachner, \& Mikulincer, 2005). Because findings relevant to the relationships among the three factors remain inconsistent, 
investigation of these factors for the American college student population is as significant as for the Taiwanese/Chinese international students.

\section{Overview: Development of Psychotherapy Theories}

The historical and current development of psychotherapy theories will be reviewed to highlight the significance of development of relational theory for the timely needs of Americans and Taiwanese/Chinese international students. Traditionally, psychotherapy emphasized the internal coping mechanisms of individuals. For instance, Freud (1922) conceptualized psychology as the internal framework developed from early infancy and then analyzed its impact on adult mental dysfunction. Behaviorism, a later development in psychology, generalized that human beings are organisms that respond to conditioning and reinforcement (Skinner, 1966). A further development in psychology, humanistic psychotherapy, focused on self-actualization and self-growth (Dennett, 1978; Locke, 1694; Maslow, 1965; Rogers, 1951, 1953). The core beliefs of humanistic psychologists about human beings are that people are unique, are capable of communicating verbally, and are self-aware. In the client-centered therapy of Rogers (1951), later renamed person-centered therapy (Bohart \& Tallman, 1999), the construct of self as well as self-consciousness creates the uniqueness of human beings and distinguishes humans from animals (Barresi, 1998). Changes and progress in psychotherapy result from the core belief in the human potential for self-exploration and self-growth. Rogers (1953) argued that human beings become persons in a process of self-growth and self-discovery. According to Rogers (1951), a fully functioning person can increase his or her self-understanding, trust his or her own capacity and potential, make choices freely, and thus live authentically.

Rogers (1964) also postulated therapist empathy as a key element in client therapeutic progress. Rogers hypothesized that individuals need to experience empathy from subjective, 
interpersonal, and objective perspectives. In addition to human needs for uniqueness, the ability to communicate, and development of self-awareness, the client need for therapists' understanding through direct communication is also considered significant (Rogers, 1964).

Similarly, Maslow $(1943,1948)$ stressed six categories of human motivation in social, physical, and intellectual needs in the present and future time. The hierarchies of these needs were conceptualized to range from basic to advanced levels of motivation in seeking satisfaction of physical needs first, followed by social needs, and finally intellectual needs with the fulfillment of present needs exceeding future needs. Social needs in Maslow's theory were hypothesized as individual affection from others and dominance (obtaining higher social hierarchy, management) needs. The significance of individual social needs, fulfilled by the perception of belongingness, friendliness, being cared about, valued, and a sense of control was again supported.

Historically, the study of interpersonal relationships has been limited in the development of theories of psychotherapy. For example, Kohut (1966) hypothesized the alter-ego-connected axis of human development in developing the ability to express emotions, and communicate with significant others, both of which are precursors to the formation of close relationships. In the development of the alter-ego-connected axis of self, infants experience dependence on the caretaker whose availability and responsiveness are essential for the development of a healthy self in the infant. This hypothesis is similar to attachment theories (Banai, Mikulincer, \& Shaver, 2005. Kohut (1971) and Stern (1980) postulated that significant others serve the role as a selfobject for infants for normal development by meeting the needs of infants and by being perceived as an extension of the infant rather than another individual. Kohut emphasized the critical role of the caretaker because the process of meeting infant needs was conceptualized to 
be a key to the learning of self-regulation for infants which in turn leads to minimizing seeking proximity and availability of the caretaker for needs in later development, such as childhood and adolescence. Similarly, Stern (1985) illustrated that infants learn self-regulation in an alteration of self-experience, created by the presence of infant (self) and the caretaker, infant seeing, hearing, feeling the caretaker, and infant feelings of belonging. This infant experience can then be utilized as a self-soothing memory, with which the infant can recall and activate the selfexperience with the caretaker and gradually be integrated by the infant into "Representations of Interactions that have been Generalized" (RIG, p. 97, Stern, 1985). According to Stern (1985), the alteration of infant subjective experience is a gradual and ongoing process, and an altering of the RIG through which infants internalize self-experience among individual episodes occurring in the surroundings. The alteration process is also considered the development of an internal working model (Bowlby, 1969; Stern, 1985). Self-object, psychoanalysis, and attachment theorists, such as Kohut, Bowlby, and Stern have all stated the significance of the interpersonal context of early experience between infants and caretakers.

As a more recent theoretical development, relational-cultural theory highlights the essential human need for connection to other people among all ethnic groups (Jordan, 2001). Jordan (2001) argued that addressing interpersonal disconnections accurately and allowing therapy to be a new and different connecting experience from other relationships could enhance interpersonal connections in general. She highlighted the significance of deep and authentic connections with others by stating that "People grow through and toward relationships throughout the life span" (Jordan, 2010, p. 24). Based on the relational-cultural theory, the effects of mutual empathy and mutual empowerment foster growth of interpersonal competence and abilities in every stage of life. Jordan further postulated that human beings suffer from 
depressive moods due to suppression of authentic feelings, which inevitably leads to isolation or disconnection from other people. According to Jordan, disconnection could result from previous negative experiences with other people, such as being rejected, criticized, or not receiving empathy. When individuals only partially engage their vulnerable selves in relationships, the level of engagement may create superficial and safer connections but further lead to feelings of being "ineffectual and helpless in shaping relationships" (p. 96). Ineffectual was defined as relationally ineffective in genuine expression of emotions and a lack of beliefs that others may value the well-being of self. Jordan highlighted that the feelings of being relationally ineffectual may elicit chronic disconnection, such as isolation, self-blame, and immobilization which can further induce characteristics of psychopathology. Thus, Jordan identified the primary task of therapy as helping the client to rebuild healing connections. The therapeutic value of therapist acceptance, support, and empowerment, which in turn promotes the client's sense of security, is conceptualized by Jordan as the foundation of effective psychotherapy. This approach clearly highlights the importance of human connection and the healing power of reconnecting to others.

\section{Cultural Differences and Impact of Acculturation}

Romantic relationships as social support for Asians. Social support has been found to be correlated with a greater subjective sense of well-being, life satisfaction, and happiness among the Taiwanese (Lu, 1995). More specifically, Lu (1995) determined that high levels of social support were determined to be associated with better psychological health and higher levels of happiness while lower levels of social support were found to be related to more psychological symptoms among urban Taiwanese adults. As Asian cultures have been generally categorized as collectivistic, individuals who reported having strong social support experienced better subjective well-being. For example, while self-efficacy, self-esteem, and coping skills may 
predict individual life satisfaction in individualistic societies, collective coping style and social support serve as strong predictors of life satisfaction for Asians who come from much more collectivistic societies (Weisz, Rothbaum, \& Blackburn, 1984).

Social support and the different impact of social support on Asians were found to be among the most important factors for subjective well-being in many studies focusing on Asian individuals. For example, in a study by Bond and Singelis (1997), which was restricted to a pool of Hong Kong participants, relationship harmony was found to be more indicative of life satisfaction than was self-esteem. Li and Liang (2007) also found that social support has a strong effect on predicting life satisfaction among elderly Chinese. Social network includes range as one subcategory. Silverman, Hecht, and McMillin (2000) defined range as the number of people in an individual's social network. Range was also found to significantly influence Taiwanese life satisfaction. Family support is another aspect of social support. Because Asian immigrants experience more stress from acculturation than non-immigrants researchers found that family support played a more significant role in Asian immigrant well-being (Kim, Hurh, \& Kim, 1993).

A significantly negative correlation has also been found between depressive symptoms and levels of social support in general. The Diagnostic and Statistical Manual of Mental Disorders IV-TR (DSM-IV TR; American Psychiatric Association, 2000) identified withdrawal from social interaction as a primary symptom of depression as lower levels of social support were determined to be strongly associated with depressive symptoms (Huang, 2009).

As a specific dimension of social support, romantic relationships also play a similar role in determining emotional functioning. For example, Beach, Dreifuss, Franklin, Kamen, and Gabriel (2008) found that depression impairs romantic love in a marriage. Given that quality of 
romantic relationships was determined to be strongly negatively correlated with severity of depression (Brown, 2000; Fagan, 2009), clinicians can choose a more effective approach for treating depressed clients if they know how attachment style, levels of depressive symptoms, and the quality of romantic relationships interact with each other. However, the reviewed studies targeted only Asian participants living in their countries of origin. Because the culture and the environment among Asian countries are different from the cultural-political environment in the United States (Kim, Yang, Atkinson, Wolfe, \& Hong, 2001), these findings may not necessarily be applicable to Taiwanese/Chinese international students in the United States.

Although social support was found to be significantly associated with decreased levels of depressive mood, Law and Sbarra (2009) found that social support was not a significant predictor for levels of depressive mood but marital status was a significant predictor. Thus, the unique effect of quality of romantic relationships on depressive moods may differ from the impact of social support in general.

Given that many Asian international students suffer from depressed moods or depression, perhaps mental health professionals should develop a "standard treatment protocol" for Asian international students with depression. The answer is negative. Asian international students do not form a homogenous population. Several Asian countries have English as their official language and were more exposed to Western culture due to long periods of colonization by Western countries, such as the former British colony, India (Anand, 2010). Although cultural differences have been observed between Asians/Asian Americans and Americans, "Asian culture" is heterogeneous. Significant cultural differences as well as coping strategies were found or observed among Asians with different countries of origin or subgroups (Hendershot, 2008; Huntington, 1996; Kim, 2010; Yeh \& Wang, 2000). For example, Filipino students were 
determined to be more acculturated than Chinese students due to their history of British colonization and the use of English as an official language (Eng, Kanitkar, Cleveland, Herbert, Fischer, \& Wiersma, 2008; Wong, 2006). Due to the value differences between Chinese (Confucianism emphasizing filial piety and education) and Filipino (moral act and gratitude), levels of acculturation contributed to different levels of student academic achievement. More specifically, levels of acculturation predicted levels of academic achievement among Filipino students, but did not have the same level of influence on Chinese students (Cheng, 1999; Eng et al., 2008).

Another example is Yeh's (2003) study focusing on Chinese, Korean, and Japanese immigrant youth. Yeh detected different levels of acculturative distress, which are affective, behavioral, academic, and psychosomatic responses to the process of acculturation among the three subgroups. Korean participants were found to endorse significantly higher numbers of mental health symptom items than did their Chinese counterparts. Different levels of acculturative stress and countries of origin, Chinese, Korean, and Japanese ethnic groups was determined to experience significant levels of mental health symptoms. More specifically, Korean immigrants endorsed statistically significantly higher levels of mental health distress than Chinese or Japanese immigrants. Higher levels of acculturative distress and lower levels of acculturation significantly predicted higher levels of general distress among these youth participants. Yeh inferred that differences in help-seeking attitudes among Korean Americans (Kim, 1996) might result in higher levels of distress among Korean American participants than Japanese American and Chinese American participants. Thus, countries of origin of international students, particularly among those for whom significant cultural differences may be 
expected, should be studied as different ethnic groups instead of single groups, such as Chinese versus Korean.

Cultural differences in interpersonal relationships. Although the general significance of interpersonal connections has been highlighted in both of the relational-cultural theory and person-centered theory, there has been much less research conducted specifically on Asian international students and immigrants. This may be due to the difficulty in recruiting participants and the smaller numbers of international students. However, significant cultural differences between the mainstream American and Asian or Asian American cultures continue to challenge psychology practitioners in providing more culturally competent service. Although the population of Asian Americans consists of international students, immigrants, and Americanborn Asian Americans, significant differences in social support and emotional adjustment according to levels of acculturation have been established among different generations of immigrants and international students (Abouguendia \& Noels, 2001). Because most Asians or Asian Americans who are second generation in the United States reported significantly higher levels of acculturation to the mainstream American culture and different interpersonal and emotional dynamics (D'Rozario \& Choudhury, 2010; Saxena, 2009), first generation immigrants and international students actually share more similar values and challenges than do second generation immigrants because of personal exposure to the countries of origin.

In addition, there is a lack of empirical study of cross-cultural differences in the correlation of the quality of romantic relationships and levels of depressive moods for Taiwanese/Chinese international students, although other cultural differences between White Americans and first generation Asian-Americans have been observed by Kim, Yang, Atkinson, Wolfe, and Hong (2001). Given the previously mentioned lack of literature to date, it is 
important to investigate the relationship between the quality of romantic relationships and levels of depressive moods as well as attachment styles and levels of depressive moods.

\section{Attachment Theory}

Attachment theory is one of the most broadly studied theories pertaining to romantic relationships. Initially, attachment styles were developed to conceptualize infant/child relationships with caregivers. Bowlby (1969/1973) proposed that infants develop attachment styles based on their interactions with their primary caregivers, who are most frequently their mothers. Ainsworth (1989), Bowlby (1979), and Cassidy (2008) further defined attachments as affectional bonds as a relatively long-term, non-interchangeable, emotionally significant inner organization with persistent individual characteristics in which individuals desire and act to maintain proximity to a particular individual. Based on hypotheses of Ainsworth (1989), the attachment system is activated when an individual separates from a specific attachment figure, such as the primary caretaker or romantic partner. When the attachment system of the individual is activated, individuals may present with proximity-seeking behaviors, such as calling to attract the attached figure to attend to or come near this individual. Emotional distress may be experienced when proximity to the attached individual is not accessible while the desire of unification and interaction as well as pleasure after unification persists. Bowlby (1977) further postulated that early attachment may be substituted in later developmental stages of a child but it frequently persists over time and the readiness of activation on the attachment system, which results in attachment behaviors (proximity seeking and distress during separation from the attachment figure), gradually faded after age three. Once the attachment system is activated by unfamiliar people, hunger, fatigue, or fear, it may then have to be deactivated by visual, tactile, 
or auditory confirmation of the proximity of the attachment figure as well as through positive interactions with the primary caretaker.

According to Bowlby (1969/1973), the sensitivity and responsiveness of caregivers toward infants are keys to the development of infant attachment styles. Caregivers who initiate social interactions, responding in a timely manner, and sensitively to infant needs are hypothesized to contribute to positive infant-caregiver relationships and secure attachment styles of infants. Securely attached infants tend to have stable moods, interact positively with people, and present less anxiety and fear when caregivers are not in close proximity (defined as reachable distance by hearing, touching, or seeing; Bowlby, 1977) to the infants. However, infants whose caregivers punish or ignore essential needs, such as eating, providing a stable environment for sleeping, and physical touch (hugging and kissing) tend to develop anxious or avoidant attachment styles with underlying fears of abandonment. Infants or children with anxious attachment styles are more likely to present more negative affect, refuse to leave parents due to separation anxiety, and exhibit other fearful behaviors (Sable, 2007). Similarly, individuals with an avoidant attachment style demonstrate negative behaviors, such as maintaining emotional distance and minimizing self-disclosure or attending to emotionally loaded interactions (Bowlby, 1977). These behaviors result in superficial attachment relationships due to fear of potential separation or abandonment by caregivers. When these individuals become adults, they tend to equate less attachment with less emotional hurt. This belief may lead to lower levels of self-disclosure, avoidance of intimate behaviors, and fear of engagement in deep conversation with partners.

After the initial development of infant/child attachment theory, research has found further that attachment experience and styles from early childhood could also contribute to adult 
attachment styles in romantic relationships. Researchers have found that adult romantic relationships and early parental love are similar (Ainsworth \& Bowlby, 1991; Hazen \& Shaver, 1994). Furthermore, Ainsworth and Bowlby (1991) hypothesized that attachment styles in romantic relationships were formed during early infant-mother interactions. According to attachment theory, parental love and attachment styles formed in infant-mother relationships serve as a cognitive behavioral system which influences attachment in romantic relationships formed later during adulthood. Those schemata or working models were used to describe automatic reactions to similar scenarios. Consistently, Curran, Hazen, Jacobvitz, and Sasaki (2007) also found that couples' emotional attunement (dyadic emotional connectedness and responsiveness) was predicted by their parental emotional attunement. Findings of Curran et al. (2007) suggested a strong connection between adult emotional attachment and emotional attachment patterns as shaped by early developmental interaction experiences with their parents. The more current research focuses on the interactions of the construct of attachment styles and depressive moods or depression, quality of romantic relationships, and culture variables.

\section{Theoretical Concepts and Early Studies concerning Attachment Styles}

Secure attachment. According to Ainsworth and Bowlby (1991), secure attachment is a pattern wherein infants generally feel safe even when distressed and seeking a parent. This attachment style allows infants to explore the world with a secure emotional status and to develop a pattern to interact with their romantic partner during adulthood with assumptions of caregiver availability and support, as defined by Bowlby (1969, 1973). Kochanska (2001) further determined that children with different attachment styles responded to stress and separation from the attachment figure with a variety of emotions. More specifically, securely attached children presented less anger over time under the stress of absence of the attachment 
figure or presence of a stranger. During adulthood, individuals with secure attachment are more likely to demonstrate confidence in developing solid romantic relationships and lower levels of anxiety and fear when romantic partners are not available or accessible (Bowlby, 1969, 1973). Securely attached individuals also can easily believe in the stability of relationships and perceive support from partners and sustain separation over time and distance during later childhood or adulthood because of the belief that the attachment figure will maintain contact and eventually return to proximity (Ainsworth, 1989; Bowlby, 1977; Fagan, 2009). Individuals tend to show behavioral patterns in interactions with the attachment figure who serves as a secure base, reflecting the beliefs in proximity maintenance of the securely attached individual described above. Likewise, Pistole (1989) and Shemmings (2006) found that persons with secure attachment reported more compromising behaviors, conflict resolution with parents, and applying more problem-solving skills during conflict with romantic partners; thus, securely attached individuals reported higher levels of relationship satisfaction than did insecurely attached (anxious or avoidant attachment) individuals.

Anxious attachment. Individuals with this attachment style tend to display attentionseeking behaviors, feelings of anxiety, and fear of loss. Individuals with anxious attachment typically worry about the unavailability of romantic partners and attempt to maintain the availability of partners. This pattern developed due to possible emotional neglect or abuse during early mother-infant interaction, and predisposes these adults to interact with their romantic partners with strong anxiety (Ainsworth \& Bowlby, 1991). This attachment style is categorized as unhealthy and insecure. Based on this theory, anxiously attached individuals are more likely to present with higher levels of anxiety and overly obsessive behaviors in seeking partner approval, seek reassurance of love, and exhibit jealousy (Collins \& Read, 1990). 
Avoidant attachment. This attachment style is categorized as unhealthy and insecure as well (Bowlby, 1969, 1973). Ainsworth and Bowlby (1991) stated that individuals with an avoidant attachment style tend to be detached to their lovers and avoid contact or react ambivalently in emotional situations. For example, Kochanska (2001) found that children with avoidant attachment presented with increased fear when the attachment figure was absent or a stranger was present. Individuals with an avoidant attachment style are generally unwilling to trust and disclose deep feelings to partners. Despite low levels of conflict, adults with avoidant attachment still present emotional distress and difficulty in emotion regulation (Allen \& Miga, 2010; Diamond \& Fagundes, 2010; Shaver \& Mikulincer, 2007). Thus, they frequently fail to attain reciprocity in communication with partners and interdependence in marriages (Feeney, 1995, 1999).

Furthermore, Bartholomew and Horowitz (1991) developed a four-factor attachment model. They labeled these factors as secure, preoccupied, dismissive and fearful. This model separated and relabeled the responses of avoidant attachment style of Bowlby (1963) into dismissing-avoidant and fearful-avoidant (or dismissing and fearful) styles. Bartholomew and Horowitz described individuals with dismissing attachment style as having positive self-concepts but negative concept of others' acceptance and warmth. More specifically, dismissing individuals tend to perceive themselves as worthy of love, but hold a negative expectation of others, such as non-accepting and non-responsive toward dismissing individuals. Individuals categorized as fearfully attached tend to hold negative views toward both themselves and others, and perceive themselves as unworthy of love and others as generally unaccepting and rejecting. Both dismissing and fearful individuals were hypothesized to choose emotional or behavioral distance under stress (Bartholomew \& Horowitz, 1991). 
Similar to the three-factor attachment model, individuals who present with or experience less emotional distress, assume the love-worthiness of self and the acceptance and warmth of others and were thus classified as securely attached (Ainsworth \& Bowlby, 1991; Bartholomew \& Horowitz). Individuals who are labeled as anxiously attached by Bowlby and Ainsworth were relabeled as preoccupied.

In order to confirm the four factor theoretical model, Bartholomew and Horowitz (1991) recruited 40 women and 37 men (predominantly Caucasian Americans 67\%, and Asian Americans 16\%; average age 19.6 years) from an introduction to psychology course. An attachment interview, a 60-minute, semi-structured interview, was designed to assess participant descriptions and feelings about significant relationships, including romantic relationships and friendships. Three raters also rated participant responses with four 9-point scales for levels of consistence with the four attachment categories (secure, preoccupied, fearful, and dismissing) when reviewing audio recordings of the attachment interviews. Levels of intimacy of friendships $(r=.77)$, involvement in romantic relationships $(r=.40)$, warmth $(r=.59)$, and balance of control in friendships $(r=.29)$ were determined to be significantly positively correlated to levels of attachment security. Dismissing individuals reported significant negative associations in their attachment rating for levels of emotional expressiveness $(r=-.69)$, involvement in romantic relationships $(r=-.35)$, warmth $(r=-.68)$, self-disclosure $(r=-.45)$, balance of control in friendships $(r=.29)$, and reliance on others $(r=-.57)$. The only significant positive association with the attachment rating was balance of control in friendships $(r=.40)$. Fearful participants indicated significantly negative association with their attachment rating in levels of involvement in romantic relationships $(r=-.36)$, intimacy in friendships $(r=-.52)$, self-disclosure $(r=-.43)$, and balance of control in friendships $(r=-.40)$ and in romantic relationships $(r=-.32)$. The only 
significant positive associated with attachment rating for fearful individuals was balance of control in friendships $(r=.40)$. Preoccupied participants indicated significantly positive correlation between attachment rating and levels of involvement in romantic relationships ( $r$ $=.34)$, emotional expressiveness $(r=.78)$, self-disclosure $(r=.64)$, reliance on others $(r=.64)$, and balance of control in friendships $(r=-.35)$ and in romantic relationships $(r=-.32)$.

Bartholomew and Horowitz's (1991) study confirmed the consistency in hypotheses pertaining to the secure and preoccupied or anxious attachment between the three-factor and four-factor models. The two different attachment styles, fearful and dismissing, were also distinctive based on their findings. Although the observer ratings may be beneficial to avoid participant defense in self-report attachment styles, solely relying on observer ratings may increase reliability issues comparing to directly assessing participant attachment by asking participants to complete valid and reliable self-report measures.

\section{Inconsistent Findings about Adult Romantic Relationships}

According to Ainsworth and Bowlby (1991), people with insecure (avoidant or anxious) attachment styles tend to have more difficulty developing healthy relationships than do people with secure attachment styles. Brennan and Saver (1998) also confirmed the theory by studying significant interactions between insecure attachment styles and 13 personality disorders. Consistent with the findings of Brennan and Saver (1998), Sable (2007) found that although insecure (anxious or avoidant) attachment styles are not classified as psychopathological, individuals with insecure attachment frequently present with symptoms meeting diagnostic criteria for mental illnesses (e.g., depression) that result in more negative affect and higher levels of distress under pressure. 
However, some researchers have reported more inconsistent findings. As an example, Dinero, Conger, Shaver, Widaman, and Larsen-Rife (2008) found that attachment security during adolescence did not contribute to romantic interactions based on a secure attachment style during early adulthood. Four hundred fifty-two, predominantly Caucasian adolescents were recruited by the Iowa Youth and Families Project (IYFP) to participate in interviews in their homes with their parents in rural Iowa from 1991 to 1992. Adolescents, aged 15 and 16 were included in two structured interviews concerning family life on issues which resulted in disagreement with their families.

Dinero et al. (2008) videotaped and trained raters (200 hours of training and maintenance weekly training) to rate family interactions using the Iowa Family Interaction Rating Scales (Melby \& Conger, 2001). Family interactions were assessed in eight dimensions, including angry coercion, antisocial behaviors, hostility, rejection, positive communication, listener responsiveness, pro-social behavior, and positive assertiveness on a 9-point scale. Structural equation models were applied to different types of relationships, including dating and cohabiting, and no significant difference was determined. Parental behaviors toward participants were found to be positively correlated with participant behaviors toward romantic partners. Dinero et al.'s findings partially supported that family interactions in an early developmental stage (adolescents, aged 15 and 16) were strongly positively associated with adult romantic interactions (age 27).

Given that most studies pertaining to attachment styles rely on self-reported measures, the strength of Dinero et al.'s (2008) study rests in an objective measure in rated family interactions. Dinero et al.’s findings were supportive of Ainsworth and Bowlby's (1991) attachment theory, but the findings may not be generalizable to ethnic minorities or international students or first generation Asian immigrants because the majority of participants were 
Caucasian Americans. More specifically, Wang (2004) and Wang and Mallinckrodt (2006) determined significant differences in ideal attachment in romantic relationships between Taiwanese and American college students and thus concluded that healthy adult attachment style was "culture-specific" (Wang, 2004, p. iv). Additionally, the data were collected only in the state of Iowa. Therefore, potential geographical differences between Iowa and other states may further limit generalizability of the findings. Because attachment theory was developed over three decades ago, most recent studies investigate the interactions between attachment styles and other variables.

\section{Interactions among Attachment Styles, Depressive Moods, and Cultural Differences}

Attachment styles and depressive moods. Shaver and Mikulincer (2007) stated that "attachment orientation or styles include a variety of cognitive, affective, and behavioral maneuvers that can alter, obstruct, or suppress the generation, activation, and expression of emotions" (p. 448). This statement drew the connection between attachment styles and emotion regulation, such as depressive moods. Researchers have hypothesized that anxious and avoidant attachment styles are associated with poor relationship quality and emotional functioning (Bowlby \& Ainsworth, 1991; Hazen \& Shaver, 1994). Empirically, Strahan (1995) determined that attachment anxiety contributed to medium level of impact on depression among college students $(\beta=.31$ for females and .39 for males), which supported the hypothesis that anxious attachment contributes to symptoms of depression. Konchanska (2001) also determined that anxiously and avoidantly attached Australian children presented with more anger and fear, but less joy in the Strange Situation scenario (Ainsworth \& Wittig, 1969), in which interactions and emotional responses of children with a stranger in the presence and absence of the primary caretaker were observed by researchers. These findings consistently confirmed the existence of a 
significant correlation between anxious attachment style and negative emotions, such as depression, fear, and anger.

Consistently, Lemmens Buysse, Heene, and Eisler (2007) and Tasca et al. (2009) determined that anxious attachment style was significantly associated with depressive symptoms. More specifically, Tasca et al. (2009) reported that attachment anxiety results in depressive symptoms after applying structural equation modeling. Lemmens et al. and Demyttenaere (2007) also found that depressed participants frequently reported anxious attachment style rather than secure attachment style. They further found that females with avoidant attachment style reported higher levels of depression than did male participants with the same attachment style. Additionally, Lemmens et al. (2007) confirmed the strong association between attachment styles and depression.

One of the few studies concerning depression, marital relationships, and insecure attachment styles is Conradi and Jonge's (2009) study. During a three-year follow-up period, 68 depressed patients who had previously received primary care completed the Beck Depression Inventory (BDI-II, Beck, Steer, \& Brown, 1996), Experiences in Close Relationships questionnaire (ECR; Brennan et al., 1998; Conradi et al., 2006), the Composite International Diagnostic Interview (CIDI, Wittchen, 1994), the Loneliness scale (De Jong Gierveld \& Kamphuis,1985), and Maudsley Marital Questionnaire (MMQ) (Arrindell et al., 1983) for levels of depression, attachment styles, baseline depressive episode, levels of loneliness, and marital problems. After converting ECR scores to four different attachment clusters (fearful, diminishing, preoccupied, and secure) based on Bartholomew and Horowitz's (1991) attachment model, Conradi and Jonge determined that individuals with fearful attachment endorsed significantly more severe depression symptoms, more depressive episodes, residual symptoms, 
longer consumption of antidepressants, and poorer social functioning than those with secure attachment. Conradi and Jonge's (2009) study provided more support for the strong association between the quality of marital relationships and depressive symptoms. However, because all participants met the criteria of major depressive disorders according to the DSM-IV, the results may not be generalizable to a non-clinical population, such as college students.

Shaver, Schachner, and Mikulincer (2005) reported relationships of depression, insecure attachment styles, and quality of romantic relationships for the college population. Shaver et al. determined the significant association between attachment anxiety and depression among 144 heterosexual undergraduate students who were mostly involved in stable, long-term romantic relationships (age 17 to 32, 45\% European Americans and 35\% Asian or Asian Americans). Both partners in the student couples completed the Experiences in Close Relationship Scale (ECR; Brennan, Clark, \& Shaver., 1998), Center for Epidemiologic Studies Depression Scale (CES-D; Radloff, 1997), Perceived Relationship Quality Component Inventory (PROQC; Fletcher, Simpson, \& Thomas, 2001), and Excessive Reassurance Seeking Scale (ERSS; Joiner \& Metalsky, 1998). Shaver et al. determined that attachment anxiety was significantly correlated to depression while excessive reassurance seeking was not statistically significant when attachment anxiety and avoidance were statistically controlled. Thus, an excessive reassurance seeking tendency was concluded to be a strongly associated factor with insecure attachment styles.

Furthermore, attachment avoidance was found to be a significant contributor $(\beta=-.72)$ of variance in the quality of romantic relationships for both male and female college students (Shaver et al., 2005) and a more significant associated factor with the quality of romantic relationship ( $r=-.76$ perceived quality, $r=-.42$ partner's perceived quality) than was attachment 
anxiety ( $r=-.33$, non-significant on perceived quality, $r=-.42$ partner's perceived quality). These findings partially support attachment theory, in which both anxious and avoidant attachment styles were hypothesized to be strongly associated with poor relationship quality.

Contrary to most other studies, Harwood (2008) found that participants with insecure attachment styles reported no significant changes in depressive affect. Harwood recruited 89 predominantly Caucasian American participants (94.4\%) who were involved in romantic relationships for at least six months. Participants' attachment styles, levels of depression, levels of anxious and depressive affect, and levels of conflict were assessed via the Experiences in Close Relationships Questionnaire (ECRQ; Brennan, Clark, \& Shaver, 1998), the Revised Conflict Tactics Scale (CTS2; Straus, Hamby, Boney-McCoy, \& Sugarman, 1996), and the Center for Epidemiological Studies-Depression Scale (CES-D; Radloff, 1977). The results did not find avoidant and anxious attachment styles to be significant predictors of depressive affect. Because Harwood's (2008) as well as Dinero et al.'s (2008) findings were inconsistent with most studies focusing on attachment styles and depressive moods, these findings raise the need to further assess the relationship between insecure attachments and depressive affect for the American population.

\section{Depressive moods and attachment styles vs. individualistic and collectivistic cultures.}

Although research has confirmed that individuals with insecure attachment styles are more likely to experience negative emotions, such as depressive or anxious moods, applicability of the attachment model of Bowlby $(1969,1973)$ on Asians remains questionable because of significant cultural differences between the United States and Asian countries. The dominant culture in the United States emphasizes individual achievement and independence while Asian cultures value social acceptance and interdependence (Mak, Bond, Simpson, \& Rholes, 2010; Sue \& Sue, 2007). 
Traditional Chinese culture can be represented by the saying, "Harmony is as treasurable as gold." Given that the social and self-construct is determined to be so different (Kitayama, Mesquita, \& Karasawa, 2006), the association with attachment styles and depressive moods may vary between these two cultures.

This cultural difference can be found in the results of a number of studies. Mak et al., (2010) used 150 Hong Kong and 201 Caucasian American college students, average age, 20.44 and 19.03, respectively, who reported an average length of romantic relationships of 23.47 and 17.12 months. Participants completed the Experiences in Close Relationships Scale (Brennan et al., 1998), the Social Support Questionnaire (Sarason, Levine, Basham, \& Sarason, 1983), and Center for Epidemiologic Studies Depression Scale (CESD, Radloff \& Teri, 1986). Consistent with both Hong Kong and U.S. samples, higher levels of perceived social support from current partners predicted higher levels of relationship satisfaction with fewer depressive symptoms. Mak et al. (2010) also determined that anxious attachment contributed to lower levels of relationship satisfaction and more depressive symptoms in both samples, but avoidant attachment was found to be predictive of lower levels of relationship satisfaction with more depressive symptoms appearing in the Hong Kong sample. Both avoidant and anxious attachment styles were detected to be predictors of lower levels of perceived social support from current partners, which further predicted lower levels of relationship satisfaction in both samples. The results yielded a strong relationship between avoidant attachment and length of romantic relationships, but length of romantic relationships did not contribute to depressive symptoms when insecure attachment variables were statistically controlled. In both U.S. and Hong Kong samples, relationship satisfaction and perceived social support with current partners served as mediators between attachment anxiety or avoidance and depressive symptoms. 
Despite the finding that relationship satisfaction and perceived social support with current partners were determined to be significant between both cultures, relationship satisfaction and anxious attachment accounted for different weights of variance for depressive symptoms (Mak, et al., 2010). Responses of Hong Kong participants were determined to account for $56.3 \%$ of the variance for depressive symptoms but responses of American participants only accounted for $36.9 \%$ of the variance. Similarly, avoidant and anxious attachment contributed $48.3 \%$ of the variance for perceived social support for Hong Kong participants, but only $12.1 \%$ for the American participants. These findings reveal that anxious attachment and relationship satisfaction with romantic partners are both associated with relational factors. Attachment avoidance was found to be more significantly associated with both relationship satisfaction and perceived support from romantic partners for the Hong Kong participants than were U.S. participants. Mak et al. thus concluded that individuals in collectivistic cultures might more likely connect to their romantic partners for relational factors, such as emotional support, and individuals with avoidant attachment may experience higher levels of distress (depressive symptoms) because of the different tendency relative to collectivistic cultural norms in acting and perceiving support. Mak et al. determined significant variables associated with depressive symptoms between Hong Kong and U.S. cultures, but did not include participants who were exposed to both Chinese and American cultures. Additionally, their study was limited to the Hong Kong area and college students, which may be significantly different from the culture in mainland China because Hong Kong was strongly influenced by the British culture due to its history as a British colony.

In yet another study, He, Zhang, Yang, Li, and Chang (2010) investigated the correlation between insecure attachment styles and social support in the central provinces of China. The 
authors found supportive results for Bowlby's model of insecure attachment styles. Perceived social support was significantly negatively associated with attachment anxiety and avoidance. Although participants with avoidant attachment reported lower levels of utility and perceived social support, how attachment styles influence individual depressive moods, psychological wellbeing or happiness was not directly measured. Thus, the relationship among attachment style, depressive moods, and quality of romantic relationship is still not clear in this study.

Similarly, Lu et al. (2009) found different levels of impact of social support on attachment anxiety and avoidance between Hong Kong and American samples. Couples dating for longer than three months, including 153 college students in Hong Kong and 210 college students in Texas, were recruited to complete the Experience in Close Relationship Scale (Breenan et al., 1998), Conflict Measure (Simpson et al., 1996), and Social Support Measurement (SSM; Sarason Sarason, \& Shearin, 1986). The Hong Kong sample completed the Chinese version and the American sample completed the English version of the three measures. Translation and back translation were provided to examine the consistency between the Chinese and English versions, and the authors reported no difference between the two versions. Lu et al. found different levels of impact from social support and conflict between the Hong Kong and American samples. Conflict was detected to positively influence attachment anxiety in the American sample, but was insignificant in the Hong Kong sample. On the other hand social support was found to be a significant predictor for lower attachment avoidance in the Hong Kong sample but non-significant in the American sample. The authors indicated that social support may be perceived positively by high conflict couples in the collectivistic culture in Hong Kong and also result in low levels of attachment avoidance. However, these authors further postulated that couples in the individualistic culture of the United States may not perceive support with the 
same positive strength as Hong Kong participants. Furthermore, high conflict couples in the U.S. were found to present with significantly higher levels of attachment anxiety than did Hong Kong couples. The authors indicated the high correlation between more conflict and less support among American couples may be due to the individualistic culture in the United States, in which independent individuals may perceive conflict with less support and present with higher attachment anxiety. The authors also stated that the concept of separation of couples as a natural outcome in the United States is not equivalent to the highly valued faith and loyalty among Hong Kong couples. Thus, conflict may lead to more anxiety about breakup among American couples.

Lu et al.'s (2009) findings supported the potentially different impact of culture on support from romantic partners and attachment anxiety and avoidance, but no depressive moods or symptoms were assessed. Additionally, despite the fact that the Hong Kong sample reported significantly different strengths among the conflict, support, and insecure attachment variables, the results may not be generalizable to Chinese or Taiwanese international students in the United States because of the differences in social-political environments between Hong Kong and the United States.

Inconsistent with the findings of Lu et al. (2009), Wang and Mallinckrodt (2006) assessed different beliefs regarding ideal adult attachment and the differences in these beliefs between Americans and Taiwanese. Undergraduate students completed the Experiences in Close Relationships Scale (Brennan, Clark, \& Shaver, 1998), and significant differences were detected between the two cultural groups, gender, and gender-culture interactions. In that study, the Taiwanese endorsed more items on the avoidant subscale as standards for healthy adult attachment than did their American counterparts, while more anxiety-provoking items were 
endorsed by Taiwanese men than American men. The findings supported possible differences in views of ideal romantic relationships between American and Taiwanese cultures.

Based on previous findings about potential cultural differences on the effect of attachment styles, Wang and Scalise (2010) confirmed that despite the finding that Taiwanese college students endorsed more items in attachment anxiety and avoidance than the predominantly Caucasian American sample (Wang \& Mallinckrodt, 2006), Taiwanese participants reported higher levels of insecure attachment and also experienced more interpersonal difficulties after applying a culturally adjusted attachment method. Two hundred and seventy-five mostly freshmen and sophomore students in a Southern Taiwan university enrolled in a Contemporary Chinese History course, which is required for all undergraduate students, completed the Mandarin version of ECR-S (Brenna et al., 1998; Mallinckrodt \& Wang, 2004) and the Inventory of Interpersonal Problems-Short Circumplex Form (IIP-SC; Soldz, Budman, Demby, \& Merry, 1995). All students who enrolled in the history course participated despite a lack of incentive. Reported ideal attachment scores by Taiwanese students were entered as a covariate in the regression equation of attachment scores from ECR-SC and interpersonal difficulty score from IIP-SC. Paired sample $t$-tests and path model analyses were also applied to determine the differences between ideal and individual attachment scores. Adjusted attachment anxiety was determined to be a significant predictor for all eight dimensions of interpersonal problems and adjusted attachment avoidance was detected to confirm the predicted outcomes on cold or social avoidant dimensions of interpersonal difficulties based on the Western model of attachment theory. Wang and Scalise's results supported the finding that attachment style predicts interpersonal problems among Taiwanese college students, but the results were limited by the college student sample in Taiwan and overall interpersonal problems. 
Although individual interpersonal problems may reflect difficulties within romantic relationships, the impact of attachment styles on the specific quality of dating or marital relationships and depressive moods of Taiwanese international students in the United States remains unclear.

Similarly, Kline, Horton, and Zhang (2008) reported differences in important factors for marriage and expressions of love between East Asian and American college students in Midwestern universities. Kline et al. found that American college students identified love, while East Asian college students identified caring, as the core of a successful marriage. Activities of expressing love were also determined to be different between the two groups. The East Asian participants reported that they express love through talking and cooking together, while the American participants reported discussing sports, cooking, and shopping together to be options for expressing love.

When ideals, core factors, and modes of expression of love are different, interpretation of the responses assessed by the instrument should be adjusted, too. Given that the components of an ideal relationship were found to be different among Americans, Taiwanese (e.g., Wang \& Mallinckrodt, 2006), and Chinese (e.g., Mak et al., 2010), the correlation between levels of depression and relationship quality might be different as well.

\section{Impact of positive and negative relationships and correlations to depressive moods}

Good relationship as a mediator of depressive moods. Research shows that higher levels of relationship quality and stability positively mediate individual management of, or recovery from, depressive symptoms. Having marital support and a good or stable marriage mediates the severity of depressive moods (Cooper, Meyer, \& Paul, 2008; Cramer, 2004; Knobloch \& Knobloch-Fedders, 2010). Cramer (2004) reported that relationship satisfaction and perceived support were positively associated. Consistently, Cooper, Meyer, and Paul (2008) 
stressed that marriage is one of the highlights in a person's life and marital support and control were found to be significantly associated with depressive symptoms (Fagan, 2009). Fagan (2009) determined that a higher level of support is associated with a decrease in depressive symptoms. In this longitudinal study, marital support was found to be related to a greater decrease in depressive symptoms in women than in men among a majority White American sample (Fagan, 2009). Fagan (2009) used the Fragile Families and Child Wellbeing (FFCW; McLanahan \& Garfinkel, 2000) which consisted of non-marital births (infants born with unwedded mothers) and 75 marital births in the matched control group in 20 cities in the United States. Fagan recruited 961 low income Hispanic, African and Caucasian couples who remained married when their children were between one and three years old and resided in the same households.

These couples completed the Composite International Diagnostic Interview-Short Form (CIDI-SF), Section A (Kessler, Andrews, Mroczek, Ustun, \& Wittchen, 1998), relationship quality items, which assessed positive and negative relationship behaviors (e.g., positive: levels of willingness to compromise, expression of love and affection, encouragement, help, listening, and understanding hurt and joy, and criticism; negative: controlling behaviors) and attitude toward traditional marriage (e.g., preference in remaining single or married). When children were three years old, mothers were found to be more likely to report increased depressive symptoms than fathers and higher levels of supportive behaviors within marriage was predictive of decreased depressive symptoms. Perceived controlling behaviors within marriage were found to be predictive of increased depressive symptoms. This study highlighted the significant relationship between marital support and decreased depressive symptoms and spousal controlling behaviors and increased depressive symptoms. However, because only Caucasian, African, and 
Hispanic American couples were included, the results may not be generalizable to the Asian population in the United States.

Marital relationships and status have also been determined to influence participant levels of depression. Herr, Hammen, and Brennan (2007) found that non-depressed men had better marital relationships than depressed men did and that their history of depression might be related to current family issues in 469 couples from a community sample in Brisbane, Queensland, Australia. Participant ages were not reported. Among women, current and past depression was associated with marital dysfunction. Herr et al. (2007) stressed that targeting current depressive symptoms in treatment may not be sufficient to resolve marital difficulties when depressive episodes reoccur. It can be concluded that targeting both the resolution of marital problems and reduction in depression, instead of focusing solely on depression, can result in better treatment outcomes for depressed individuals.

Beyond the direct impact of relationship quality on levels of depression, the mediation effects of martial support were also determined (Proulx, Buehler, \& Helms. 2009). Proulx et al. found that marital warmth, a significant factor in relationship quality, mediated the correlation between levels of spousal hostility and depressive symptoms. In other words, supportive behaviors of husbands and wives were found to reduce the impact of hostile behaviors on increased levels of depression.

Furthermore, stability in a relationship has been shown to decrease individual symptoms of depression (Brown, 2000). Brown (2000) reported that the number of Americans who experienced depression was significantly exacerbated by relationship instability. More specifically, Brown reported that non-married people who cohabited reported higher levels of depression than were reported by married individuals. She concluded that the increased levels of 
depression among those who cohabited accounted for the instability of the relationships, particularly for long-term cohabiters. Consistent with this conclusion, Cooper, Meyer, and Paul (2008) confirmed that a stable relationship, such as marriage or committed cohabitation, contributed to lower levels of depression in the United States, Australia, the Netherlands, and England. Cooper et al. surveyed 538 non-clinical participants, aged 20 to $82(M=39)$ years in the United States, Australia, the Netherlands, and England. The participants who identified "testing" as the reason for cohabiting experienced more relationship instability than was reported by participants who identified other reasons for cohabitation. As would be expected, those individuals who reported cohabiting as a means of testing the relationship also reported higher levels of depressive symptoms (Cooper et al.). The negative effect of being unmarried on increased depressive mood was also determined by Law and Sbarra (2009) in a longitudinal study over eight years. The study was based on data from the Australia Longitudinal Study of Aging (ALSA), and involved participants over 70 years old who resided in a Metropolitan area in South Australia. Participants reported that the status of "getting married" was determined to predict decreased levels of depression, while "remaining unmarried" was found to be predictive of significantly increased levels of depression. Thus, stability of romantic relationships and its predictive value for levels of depression was confirmed again.

Marital conflict and depressive moods. Conversely, poor marital quality was determined to be associated with more depressive symptoms (e.g., Beach, Dreifuss, Franklin, Kamen, \& Gabriel, 2008; Herr, Hammen, \& Brennan, 2007). Beach et al. (2008) reviewed relevant research and concluded that depressed couples had more difficulty in resolving marital problems than did more emotionally healthy couples. They stated that a depressed individual might express more negative affect or interact negatively with his or her partner. Consistently, 
Herr et al. (2007) found a stronger correlation between poor marital functioning and depression in females than males. Pruchno, Wilson-Genderson, and Cartwright (2009) surveyed patients with chronic diseases and their spouses and found that depressive symptoms were negatively correlated to marital satisfaction of both the patients and their spouses. Hence, individuals experiencing depression might be expected to encounter more marital problems than their nondepressed counterparts. Similarly, Lemmens, Buysse, Heene, Eisler, and Demyttenaere (2007) reported that depressed participants and their partners reported less satisfaction in marriage than the control group, non-clinical sample recruited via local media and public service announcements (newspapers, radio and television appeals).

Likewise, Knobloch and Knobloch-Fedders (2010) found that depressive symptoms contributed to perceived relationship quality. Relational uncertainty was defined as the levels of confidence in perceived involvement in the sphere of interpersonal relationships (Knobloch, 2007a; Knobloch \& Solomon, 1999, 2002a). Self-report data were collected from 170 romantic partners $(n=85$ dyads $)$ and relational uncertainty was determined to be a mediator of depressive symptoms and relationship quality. Participants completed the Beck Depression Inventory (BDI1A) (Beck \& Steer, 1993), Dyadic Adjustment Scale (DAS; Spanier, 1976, 1988), global distress subscale of the Marital Satisfaction Inventory-Revised (MSI-R; Snyder, 1997), and brief versions of Knobloch and Solomon's (1999) scales assessing feelings of uncertainty of self and perceived partner uncertainty toward relationships. Responses from female participants revealed a positive association between both depressive symptoms-relationship uncertainty and depressive symptoms-relationship distress (Knobloch \& Knobloch-Fedders, 2010)

Marital conflict has been found to be positively associated with levels of depression (e.g., Choi \& Marks, 2008; Kouros, Merrilees, \& Cummings, 2008). Kouros et al. (2008) detected that 
both depressed men and women reported engaging in higher levels of conflict and physical aggression than did non-depressed individuals. Choi and Marks (2008) reported that marital conflict resulted in increased levels of depressive symptoms in adults in the community. They also determined that marital discord directly contributed to higher levels of depression and accompanying functional impairment. Marital conflict indirectly contributed to increased levels of depression.

Researchers have also found that hostility from husbands and wives was correlated differently with the depressive symptoms of their partners. Proulx, Buehler, and Helms (2009) detected a strong correlation between husbands' hostility and higher levels of wives' depression in 419 parents of sixth-grade students in a southeastern county. Notably, warmth, supportive behaviors or expressions of emotional support for spouses reduced the impact of husbands' hostility on wives' levels of depression. When couples perceived more warmth from their spouses, the correlation between husbands' hostility and wives' levels of depression was less pronounced than in couples who perceived less warmth. Hostile behaviors of husbands were positively associated with increased levels of depressive symptoms among wives. Similarly, Lemmens et al. (2007) detected that clients with depression reported lower levels of marital satisfaction and higher levels of attachment difficulties. More avoidant communication was determined among couples with depression than the non-clinical sample.

Conflict was determined to mediate relationship satisfaction or depression among most studies (e.g., Choi \& Marks, 2008). However, Proulx et al. (2009) found that levels of depression of husbands were not significantly associated with hostile behaviors of wives. Given the different findings among reviewed studies, further investigation may be needed to determine the relationship between levels of depressive symptoms and relationship factors. 


\section{Methodological Limitations}

Generalizability is the most salient issue limiting broad applicability of the reviewed studies. Accuracy in generalizing existing findings pertaining to attachment styles with current romantic partners to the Taiwanese/Chinese international student sample may be questionable. Generalizability to an Asian sample or Taiwanese/Chinese sample is a common issue among reviewed studies. Given that participants of most reviewed studies were predominantly Caucasians, most findings may not be generalizable to non-Caucasians. Only a few studies included ethnic minority populations as participants. Given the significant cultural differences found between American and Chinese/Taiwanese cultures (Kim, 2010; Kim, Atkinson, \& Yang, 1999; Raghavan, 2006; Sue \& Sue, 2003, 2007), potential differences between factors related to depressive moods and romantic relationships may exist. Although the findings from studies targeting Taiwanese or Chinese samples may be more generalizable to Taiwanese/Chinese international students in the United States, the impact of American culture, adjustment issues, and environmental factors in the United States (e.g., different interpersonal dynamics, communication styles, languages, legal, and educational systems) and the applicability of existing research may still be an issue for this particular population.

Additionally, the applicability of findings of reviewed studies including Chinese/Taiwanese students in Taiwan/China or international students may be limited to participant beliefs of ideal attachment styles for the general population instead of assessing selfreport individual attachment styles. For instance, several studies assessed participant report of ideal attachment styles in the general population (e.g., Wang \& Mallinckrodt, 2006; Wang et al., 2007) instead of considering attachment dimensions with current dating partners or spouses. Applying participant selection criteria to participants who are currently involved in romantic 
relationships (dating relationships or marriage) and requesting participants to respond to an attachment measure according to current experience may more accurately assess individual attachment styles.

Although most reviewed studies recruited undergraduate students or clinically depressed samples, little research has targeted the non-clinical college student body with the inclusion of graduate students. Furthermore, no research has compared the differences between individuals currently involved in dating relationships and those in marriage.

\section{Rationale for the Research}

Though marriage was found to be important when treating depressed clients, the precursor to marriage, i.e., a dating relationship, was lacking in the literature. Little research has addressed the relationships between levels of depressive moods, attachment styles, relationship quality, and/or cultural differences for these constructs.

Given the cultural differences among Asian countries, Leong and Gim-Chung (1995) recommended narrowing targeted samples to attain higher homogeneity among participants for more meaningful research results. Thus, targeting a sample which consists of participants from a similar culture and speaking the same language may more accurately assess participant experience and reduce confounding factors arising from ethnic differences among Asian countries. Given the growing and large numbers of Chinese international students, who share a similar culture and the same official language with Taiwanese international students, an inclusion of both Chinese and Taiwanese international students in this study should maintain sufficient homogeneity of the targeted sample.

Moreover, cultural differences in the correlations between depressive moods, attachment styles, and quality of romantic relationships between Americans and the Taiwanese/Chinese 
students and immigrants in the United States have not yet been studied. Differences such as language, customs, and cultures among Asians also suffer from a lack of relevant research study. Research should investigate the correlations between attachment style, relationship quality, and severity of depressive moods. Because different experiences between Americans and Taiwanese/Chinese students and immigrants may lead to different relationships of attachment style, and levels of depressive moods on relationship quality between these two populations, the interactions of these factors should also be further studied. Such a study would be significant because as previously noted few studies have focused on the interactions among severity of depressive moods, quality of relationships, attachment styles, and the different experiences of the two cultural groups. Accordingly, practitioners can utilize the findings of such research to become more culturally competent in treating the Taiwanese/Chinese population in the United States and be more aware of cultural differences between this population and Americans.

Thus, the purpose of this study is to investigate levels of depressive moods and quality of romantic relationships among participants with different attachment styles between Americans and Taiwanese/Chinese international students in the United States. This study will explore the potentially different experiences of American participants and participants from the Chinese/Taiwanese culture. Furthermore, because first generation Asian Americans may experience a lower level of acculturation and different impact of attachment styles on quality of romantic relationships than second generation Asian Americans, it is important to further investigate the association between depressive moods, quality of romantic relationships, and attachment styles between American and Taiwanese/Chinese international students. 


\section{Research Questions}

To date there has been little research into differences in the relationships between attachment styles and relationship quality, or depressive moods between American and Taiwanese/Chinese individuals. Therefore, attachment styles, levels of depressive moods, and the quality of romantic relationships will be explored in this dissertation research.

Research Question 1. Is there a statistically significant difference in self-reported levels of depressive moods for American college students with attachment avoidance when compared to self-reported levels of depressive moods for Taiwanese/Chinese international students with attachment avoidance?

Research Question 2. Is there a statistically significant difference in self-reported levels of the quality of romantic relationships for American college students with attachment avoidance when compared to self-reported levels of the quality of romantic relationships for Taiwanese/Chinese international students with attachment avoidance?

Research Question 3. Is there a statistically significant difference in self-reported levels of depressive moods for American college students with attachment anxiety when compared to self-reported levels of depressive moods for Taiwanese/Chinese international students with attachment anxiety?

Research Question 4. Is there a statistically significant difference in self-reported levels in the quality of romantic relationships for American college students with attachment anxiety when compared to self-reported levels of depressive moods for Taiwanese/Chinese international students with attachment anxiety? 
Research Question 5. The following set of four research questions are all related to whether the linear combination of two dependent variables (levels of relationship quality and depressive moods) is statistically significantly different between American college students and Taiwanese/Chinese international students with different attachment categories (secure, preoccupied, dismissive, and fearful).

Research Question 5A. Are levels of relationship quality statistically significantly different among Taiwanese/Chinese international students with different attachment categories?

Research Question 5B. Are levels of relationship quality statistically significantly different among Taiwanese/Chinese international students with different attachment categories?

Research Question 5C. Are levels of depressive moods statistically significantly different among American students with different attachment categories?

Research Question 5D. Are levels of depressive moods statistically significantly different among American students with different attachment categories? 


\section{CHAPTER TWO \\ METHODOLOGY}

The purpose of this study was to investigate the differential effect of insecure attachment styles on the quality of romantic relationships and levels of depressive moods between American and Chinese/Taiwanese college students.

\section{Participants}

The target population for this dissertation was international students from Taiwan or China and American students who were enrolled in undergraduate and graduate programs. The selection criteria for the Taiwanese/Chinese sample were 1) international students from Taiwan or China (self-identify as Taiwanese or Chinese, born, and raised in Taiwan or China) currently enrolled in a university or college for undergraduate or graduate level studies in the United States, 2) involved in a dating relationship, cohabitation, or marriage when participating in the study, and 3) ranged in age between 18 and 40. The selection criteria for the American sample were 1) students enrolled in a university or college for undergraduate or graduate level studies in the United States when participating in the study, 2) involved in a dating relationship, cohabitation, or marriage when participated in the study, 3) identified as Americans (born and raised in the United States, second generation or beyond), and 4) ranged in age between 18 and 40.

A power analysis was conducted to determine target sample size, factoring in a .80 power level at an alpha level of .05. More specifically, Cohen's $d$ was used to calculate estimated sample size. Standard deviations of .35 for depressive moods and .21 for quality of relationships were entered based on existing literature, such as studies by Barth et al. (2009), Wang and 
Ratanasiripong (2010), Wang and Scalise (2011), and Yeh (2003). Analysis using IBM SPSS software version 19.0 revealed an estimated sample size of 120 participants.

Due to the necessity for at least 120 participants who would meet the selection criteria at power level .80, both random cluster and snowball sampling was applied to recruit participants. These methods were employed because of levels of representation of the target population gained by random cluster sampling, limited resources in conducting random sample, the relatively small numbers of international students from Taiwan or China, and the accessibility of student samples through student associations or student governments. Random cluster sampling is described as "sampling in which a random process is used in selecting a number of different clusters of elements from the population of interest" (Macready, 2005, p. 89). In this research, the sampling method was applied by randomly selecting 30 United States universities from 207 on the lists of Research Universities (very high research activity) and Research Universities (high research activity) on the website of Carnegie Classification of Institutions of Higher Education (Carnegie Foundation of Excellence Teaching, 2010). Universities with high levels of research activities were chosen because they appeared to attract more international students due to the reputation. Additionally, a liberal number of sample universities was decided to ensure enough recruitment of international students.

Data then was collected from existing organizations within the chosen university (e.g., international student offices, student associations, or student governments). Additionally, snowball sampling refers to participant recruitment by requesting research participants to forward information regarding research recruitment to members of their social networks. With this method, participants were asked to forward the email for research recruitment to friends or classmates who meet the selection criteria for this research. Snowball sampling was employed to 
ensure sufficient numbers of participants, particularly international students from Taiwan or China.

\section{Procedure}

This study first was submitted through the Institutional Review Board (IRB) at West Virginia University to obtain approval. After the IRB approval was obtained, informed consent (Appendix C) with a short description of participation procedures, contact information for this researcher, confidentiality of individual test results, anonymous participation, and information about the lottery incentive were provided (online or on flyers) to participants before they complete the research questionnaires. After participants were informed about all procedures for completing the instruments and agreed to participate, they were required to sign the informed consent and receive a copy of the consent before any research proceeded. Individual participants completed four questionnaires online: (a) The Quality of Relationships Inventory (QRI; Pierce, Sarason, \& Sarason, 1991; Appendix E), (b) Experiences in Close Relationship Scale (ECR-R; Wei et al., 2007; Appendix F), (c) the Center for Epidemiologic Studies Depression Scale (CESD; Radloff, 1977; Appendix G), and (d) a demographic sheet (Appendix D) with six open-ended questions. Each of these instruments is described more fully in the following section.

Participants were contacted by sending an email request to the staff, faculty or member responsible for disseminating information to students of individual academic departments, international student offices, or other student organizations, to forward the invitation (Appendix A) to the listserv or a group email list created by the initially contacted person for contact or announcement purposes. The request email described the requested distribution method (via an email and flyer), purpose of the study, procedures of participation, incentives, and contact information for this dissertation author and advisor. The invitation and a flyer (Appendix B) for 
student participants were also attached to the email. Invitation and flyers were designed to be posted at announcement boards of the contacted offices or forwarded to potential participants. Additionally, the same invitation was posted by this dissertation author on existing Facebook, Yahoo, or MSN groups of American and international student associations of the 30 selected universities. The electronic link and the online student groups were not associated. Thus, the confidentiality of participants was ensured. The invitation and flyer both described the purpose of the study, procedures of participation online on Google Docs, incentives, and contact information for this dissertation author and advisor. Interested participants could choose the website link attached to the invitation or flyer. The informed consent form was presented to participants first. After participants agreed to participate in this study on Google Docs, they could proceed to complete the three measures for depressive symptoms, attachment styles, and quality of romantic relationships, as well as one demographic questionnaire. After participants completed the four questionnaires online, they were given the option of providing their email address to this author in order to enter the drawing for two Amazon gift cards. Participants who wished join the drawing for an incentive provided their email address after completion of all questionnaires. They were informed that their email address would not be associated with their responses and the email would only be used for the incentive drawing. A drawing among all participants who provided their email address was conducted after data collection was completed. Each of the two winners were contacted by email and a \$30 Amazon gift card was sent via mail to the mailing address provided to this author.

\section{Instruments}

This study employed three standardized measures to assess participant levels of depressive symptoms, attachment styles, and quality of romantic relationships, as well as one 
demographic questionnaire to obtain information about participant demographics. The four measures were: (a) The Quality of Relationships Inventory (QRI; Pierce, Sarason, \& Sarason, 1991), (b) Experiences in Close Relationship Scale (ECR-R; Wei et al., 2007), (c) the Center for Epidemiologic Studies Depression Scale (CES-D; Radloff, 1977), and (d) a demographic sheet with six open-ended question.

\section{Quality of Relationships Inventory (QRI; Pierce, Sarason, \& Sarason, 1991;}

Appendix E). The QRI is a 25-item, self-reported, 4-point Likert scale (from 1-not at all, 2-a little, 3-quite a bit, to 4-very much very much), which is used to measure the perceived quality of love. The total score ranges from 25 to 100 points. The QRI comprises three subscales: 1) social support, (seven items, e.g., "To what extent could you count on this person for help with a problem?”), 2) depth (six items, e.g., "How positive a role does this person play in your life?"), and conflict (12 items, e.g., "How often does this person make you feel angry?"). Verhofstadt, Buysse, Rosseel, and Peene (2006) evaluated the factor structure of the QRI, and three models of the QRI were assessed with a confirmatory factor analysis. These confirmed that the QRI measures constructs of support, conflict, and depth of romantic relationships. The sample included 572 individuals from 286 couples. Invariance of gender and quality of relationship were analyzed. In the confirmatory factor analysis, the three inter-correlated model (conflict, support, and depth in romantic relationships) was determined to be best supported by the data. Internal consistency reliability (Cronbach's $\alpha$ ) ranged from .79 to .88 and was determined for the three factors in both male and female groups, indicating the reliability of subscales for both of the male and female groups. Responses for the depth subscale were found to be positively associated with the support subscale $(r=.726)$ while responses for the support subscale were determined to be negatively correlated to the depth subscale ( $r=-.437$; Pierce et al., 1991). 
Consistently, in Verhofstadt et al.'s model, conflict, support, and depth of romantic relationship variables were correlated to one another. More specifically, moderate to strong levels of positive inter-correlations were determined between support and the depth variable $(r=$ .63 for males; .77 for females) while conflict and support ( $r=-.57$ for males; -.63 for females) as well as depth and conflict ( $r=-.50$ for males' -.58 for females) were found to be negatively correlated. Most of the 25 items in the QRI contributed to strong factor loadings ranging from .52 to .74 , support (ranging from .49 to .83 ), and conflict (ranging from .43 to .87 ) constructs and gender invariance was determined. In other words, most of the QRI items demonstrate strength for the support and conflict constructs and the assessment of the whole concept of relationship quality.

\section{Experiences in Close Relationship Scale-Short Form (ECR-S; Wei, Russell,} Mallinckrodt, \& Vogel, 2007; Appendix F). Among attachment measures, the Experiences in Close Relationship Scale (ECR; Fraley, Waller, \& Brennan, 2000) is among the commonly used measures. However, considering the effectiveness in sampling and the fatigue factor (a threat to internal validity) for participants, the ECR-S (Wei et al., 2007) was used in this study.

Shortening participant completion time can reduce the validity threats of fatigue and random responses. The ECR-S (Wei et al., 2007) was adapted from the commonly used measure for attachment styles, Experiences in Close Relationships (ECR; Fraley et al., 2000). The ECR-S consists of 12 items with a 7-point Likert scale from 1-strongly disagree to 7-strongly agree, measuring insecure (anxious and avoidant, six items of each subscale) attachment styles. The attachment anxiety score comprises the sum of six different items for anxiously attached experience with reverse scoring rules (e.g., "I need a lot of reassurance that I am loved by my partner."). The score for avoidant attachment also comprises the sum of six items for avoidant 
attachment (e.g., "I try to avoid getting too close to my partner.”). Wei et al. (2007) reported coefficient alphas of .78 (Anxiety) and .84 (Avoidance) in the college student population. A low correlation $(r=.19)$ between the Anxiety and Avoidance subscales was reported by Wei et al., indicating that the two subscales measure two different attachment styles. In these authors' factor analyses, six items contributed to the Anxiety factor and six items contributed to the Avoidance factor. One-month test-retest reliability ranged from .80 to .82 and showed a statistically significant difference between the ECR and ECR-S (Cohen's $d \mathrm{~s}=-.10$ to .03 ). No statistical difference of means of attachment anxiety and avoidance subscales was found at retest. These results yielded an acceptable reliability of the ECR-S.

\section{Center for Epidemiologic Studies Depression Scale (CES-D; Radloff, 1977;}

Appendix G). The CES-D is the most broadly used measure for research that focuses on depression in the general population. The CES-D assesses four components of depressive moods, including depressed affect, positive affect (reversed scoring), somatic complaints, and interpersonal experience. Since the development of CES-D in 1977, a number of researchers have confirmed the applicability of the use of this measure to assess depressive symptoms in the general population and results on the CES-D remain consistent with depression symptoms as listed in the DSM -IV-TR. (e.g., Kim, DeCoster, Huang, \& Chiriboga, 2011; Ross \& Mirowsky, 1984). The depressed affect component refers to feelings of sadness, the blues, and irritation ("feel bothered"), while the positive affect component includes happiness, joy, and hope. The somatic component consists of items assessing physiological aspects of depression, such as depressive moods, and somatic complaints (loss of appetite, sleep disturbance, and experiencing difficulty to "get going"). The interpersonal experience component of the CES-D assesses the interpersonal effects of depression, such as "People were unfriendly" or "I felt that people 
disliked me." The instrument consists of 20 self-report items with a four-point scale from 0 rarely or none of the time (less than one day), 1-some of a little of the time (1-2 days), 2occasionally or a moderate amount of the time (3-4 days), or all of the time (5-7 days) during the past week. For example, "I felt depressed" assesses depressive affect, "I felt hopeful about the future" assesses positive affect, "My sleep was restless" assesses the somatic component, and "I felt that people disliked me" assesses the interpersonal experience of depressive moods (Radloff, 1977).

Radloff (1977) developed this measure by including items relevant to depressive symptoms for content validity, testing the correlation between CES-D and existing valid selfreport depression scales and clinical ratings, and scores for clinical and general populations for criterion-related validity. The original norming samples consisted of 1971 participants, with more than $79 \%$ Caucasian Americans. The sample included community samples of Caucasian Americans residing in Missouri, Maryland, and Connecticut, as well as a clinical sample of resident patients in a Missouri psychiatric facility. Radloff reported significant differences between the scores of all community and psychiatric participants $(t=9.36, p<.001)$. Reliability of the CES-D was also determined by the strong internal consistency identified in the community and clinical samples (coefficient alpha $\approx .85$ in the general sample, $\approx .90$ in the clinical sample). The percentage of participants endorsed higher than the 16 cutoff point for clinical depression was found to be $21 \%$ in the general sample and $70 \%$ in the clinical sample. Moderate levels of test-retest correlations were determined after 2, 4, 6, 8 weeks, and 3, 6, and 12 months after the initial assessment, ranging from .49 to .67. Only one test-retest correlation was lower than $.49(r$ $=.32)$. 
Supporting the validity and sensitivity for depressed and non-depressed samples of the CES-D, moderate correlations were found by Radloff (1977) between the CES-D and the Hamilton Clinician's Rating scale $(r=.44)$, the Raskin Rating scale $(r=.54)$, and clinical ratings of depression by the nurse clinician $(r=.56)$. In addition, a negative correlation $(r=-.55)$, between CES-D and the Bradburn Positive Affect scale was found and a strong positive correlation was detected between the CES-S and the Bradburn Negative Affect scale $(r=.55$ .63). Participants who indicated "need for help (psychiatric service)" also endorsed significantly higher scores on the CES-D. Factor loadings for the depressed, positive affect, somatic, and interpersonal components were found to range from .59 to .83 .

Since Radoff's initial findings reported in 1977, many studies have attested to the validity and reliability of CES-D (e.g., Kim, DeCoster, Huang, \& Chiriboga, 2011; Ross \& Mirowsky, 1984). Considering the purpose of this dissertation research, only studies that validated the CES$\mathrm{D}$ in the population relevant to this study, American college students and Taiwanese or Chinese populations, were reviewed. The CES-D has been used in a number of other studies in the United States, Taiwan, or China. For example, Herman, Archambeau, Deliramich, Kim, Chiu, and Frueh (2011) recruited students in introductory psychology courses to complete the CES-D. Students who completed the CES-D identified themselves as European American, Asian American, Native Hawaiian, or Pacific Islander. No statistically significant difference was determined among the ethnic groups. Furthermore, strong internal consistency reliability coefficients were $.88, .83, .77, .68$, and .55 for the CES-D scores.

Yen, Robins, and Lin (2000) administered the CES-D to Caucasian Americans, Chinese Americans, and Chinese community and outpatient samples as well as undergraduate students and detected a significant main effect of ethnicity $(\mathrm{F}(2,293)=10.46, p=.001)$. More 
specifically, the Chinese group endorsed significantly lower somatic scores than the Caucasian American group $(\mathrm{F}(197)=-4.84, p=.001)$ and the Chinese American group, $t(195)=-2.71, p=$ .007). Additionally, Yen et al. determined a non-significant difference between the somatic scores of Chinese American and the Caucasian American participants $(t(196)=1.75, p=.08)$. Yen et al.'s findings of non-significance between the CES-D scores of Chinese Americans and Caucasian Americans confirm the acceptability of inclusion of Chinese Americans and Caucasian Americans in the same group in this dissertation study.

The CES-D was also frequently used for research purposes in the Chinese or Taiwanese population. Chi and Chou (2001) determined a significant negative correlation between the CES-D and strength of the social network and perceived social support, such as number of close relatives and close relatives seen in the past month, ranging from -.14 to -.24 . Chi and Chou's findings suggested the conformity between CES-D and the diagnostic criteria of social isolation in DSM-IV-TR. Similarly, Song et al. (2008) recruited 988 college freshmen in Hong Kong and 802 in Beijing and applied the CES-D to assess the levels of depression and determined significantly different CES-D scores between depressed and non-depressive students ( $\mathrm{M}=23.42$ vs. 7.65 in Beijing and 24.48 vs. 8.88 in Hong Kong), which supported the sensitivity of CES-D to distinguish the depressed and non-depressed Chinese samples. Chi and Boey (1993) found strong levels of internal consistency (Cronbach's $\alpha$ of .85 for a community population and .90 for a clinical population) in the Chinese population in Hong Kong.

Concerning the use of online and paper forms of the CES-D, Yu and Yu (2007) tested 2,400 grade school teachers in Taiwan and determined invariance of the CES-D between online and paper forms. The depressed mood, positive affect, and interpersonal components were found to be statistically insignificant between participants in the online and paper groups. Only the 
somatic component was significantly different between the two groups at alpha level .01. However, Yu and Yu reported a small effect size (Cohen's $d=0.172$ ) and thus concluded that no meaningful interpretation for the difference of somatic component could be made. A confirmatory factor analysis (CFA), a form of factor analysis designed to test whether measures of a construct fit the structure of a model, was used. Significant factoring loadings were detected, ranging from .75 to .97 and - .65 (positive affect), which suggests an overall acceptable model of fit. In other words, the detected factor loadings confirmed the four factor structure (positive affect, depressed affect, somatic, and interpersonal factors) of the CED-S.

Thus, the reviewed literature supports the moderate to strong validity and reliability of the CES-D when applied to the Taiwanese and Chinese populations as well as to American college students. The invariant findings of $\mathrm{Yu}$ and $\mathrm{Yu}$ also supported the applicability of the two methods (paper and online) of using the CES-D as proposed in this dissertation study.

Demographic Sheet (Appendix D). The demographic sheet assesses age, gender, state location, ethnicity, level of current degree program, length of time in the United States, country of origin, length of romantic relationship, status of relationship (dating, co-habilitation, and marriage), ethnicity of partner, employment status, and number of children. The information was used in post-hoc analyses for additional information in understanding the results. Six openended questions are also included to explore participant thoughts about factors that impact relationship quality and the influence of culture and interactions with a partner on moods and relationship quality. These questions were designed to assess the differences between Taiwanese/Chinese and Americans in quality of romantic relationships, levels of depressive moods, and attachment styles. The six open-ended questions, which can also be found in Appendix D are: 1) What factors do you think impact relationship quality with this romantic 
partner? 2) How do these factors (in question one) impact your relationship quality with this romantic partner? 3) How does your cultural background impact your current romantic relationship? 4) How does the American culture cultural impact your current romantic relationship? 5) What are your taboos when communicating with your romantic partner? 6) From your point of view, how do you and your interactions with your partner impact your relationship quality and moods?

\section{Data Analysis}

The frequency, means, and standard deviations of participant descriptive information were calculated regarding participant age, gender, ethnicity, the status of romantic relationship, and country of origin. Then the statistical analyses as they correspond to each hypothesis are described.

Descriptive statistics were presented for the participant characteristics of age, gender, ethnicity, country of origin, and status of relationship. Means, standard deviations, and percentages within the American and Taiwanese/Chinese samples of depressive moods, attachment styles, and relationship quality scores were calculated with IBM SPSS software version 19.0. After calculating descriptive statistics of participant characteristics, the hypotheses were tested with independent samples $t$-tests, multivariate analysis of variance (MANOVA), and follow-up analyses of variance (ANOVAs). The rationale for the choice of these statistical analyses is explained.

Inductive (bottom up) thematic analysis with a semantic approach (identify explicit meanings of the data) was used to analyze qualitative data (Boyatzis, 1998; Braun \& Clarke, 2006). The thematic analysis of Boyatzis (1998) was applied to identify themes associated with depressive moods, attachment styles, romantic relationships, cultural effects, and taboos. 
First, the dissertation author familiarized with the data by reading the qualitative responses several times to develop a general understanding. Then the author identified prevalently appearing key words among the responses and generating initial codes based on these key words. Then the author searched for themes which are relevant to the research topics (romantic relationships, cultural effects, depressive moods, and attachment styles) among the generated codes. Major themes were reviewed after they are identified to ensure the relevancy between themes and codes. Then the author continued to refine the themes during the analysis, labeled (name) and defined each theme. At the end, the author summarized the themes and integrated with quantitative findings.

Because this author is a Taiwanese international student and has more understanding in the collectivistic, general Chinese culture, there may be potential bias against the American culture due to her background. However, given that a semantic approach was used and the qualitative data will solely be used as supplemental information for the quantitative findings, the potential bias might be reduced by avoiding interpretation of the data beyond participant statements and centering the focus of this study on more the objective quantitative findings. Additionally, bracketing was also applied during the analysis of the qualitative data (Fischer, 2009). This author read and coded the data based on participant responses instead of the author's assumptions. Then the author reviewed these codes and re-labeled themes when noticing themes which reflected personal assumptions or values.

Before conducting the statistical analysis, participant scores higher than the mean of attachment avoidance scores were transformed into two (high level of avoidance) and those lower than the mean attachment anxiety were transformed into one (low level of avoidance). 
The same method was used to transform the attachment anxiety scores. Because interval data were collected for the dependent variables (QRI and CES-D), these scores remained the same.

Hypothesis 1. There will be a statistically significant difference in self-reported levels of depressive moods for American college students with attachment avoidance when compared to self-reported levels of depressive moods for Taiwanese/Chinese international students with attachment avoidance.

The select cases function on SPSS was used to select only participants with high levels of attachment avoidance for this analysis. An independent samples $t$-test was conducted to determine if there is a significant difference in levels of depressive moods (CES-D scores) between Taiwanese/Chinese international students and American students with high levels of attachment avoidance (scores of the attachment avoidance subscale in ECR-S).

Hypothesis 2. There will be a statistically significant difference in self-reported levels of relationship quality for American college students with attachment avoidance when compared to self-reported levels of relationship quality for Taiwanese/Chinese international students with attachment avoidance.

The select cases function on SPSS was used to select only participants with high levels of attachment avoidance for this analysis. An independent samples $t$-test was conducted to determine if there is a significant difference in levels of relationship quality (QRI scores) between Taiwanese/Chinese international students and American students with high levels of attachment avoidance (scores of the attachment avoidance subscale in ECR-S).

Hypothesis 3. There will be a statistically significant difference in self-reported levels of depressive moods for American college students with attachment anxiety when compared to self- 
reported levels of depressive moods for Taiwanese/Chinese international students with attachment anxiety.

The select cases function on SPSS was used to select only participants with high levels of attachment anxiety for this analysis. An independent samples $t$-test was conducted to determine whether there is a significant difference in levels of depressive moods between Taiwanese/Chinese international students and American students with high levels of anxiety (scores of the attachment anxiety subscale in ECR-S).

Hypothesis 4. There will there be a statistically significant difference in self-reported levels in the quality of romantic relationships for American college students with attachment anxiety when compared to self-reported levels of depressive moods for Taiwanese/Chinese international students with attachment anxiety.

The select cases function on SPSS was used to select only participants with high levels of attachment anxiety for this analysis. An independent samples $t$-test was conducted to determine if there is a significant difference in levels of relationship quality (QRI scores) between Taiwanese/Chinese international students and American students with high levels of attachment anxiety (scores of the attachment anxiety subscale in ECR-S).

Hypothesis 5. The linear combination of two dependent variables (levels of relationship quality and depressive moods) will be statistically significantly different between American college students and Taiwanese/Chinese international students with different attachment categories (secure, preoccupied, dismissive, or fearful; Bartholomew \& Phillip, 1998).

Before testing the main hypothesis with a MANOVA, assumptions of sample representativeness, sample size, levels of measurement, normality of the distribution, 
homogeneity of variances, linearity relationship between dependent variables, homogeneity of variance-covariance matrices, and multi-collinearity was evaluated (Abu-Bader, 2010).

Based on the same method of previous studies, responses on the Experiences in Close Relationship Scale (ECR; Fraley, Waller, \& Brennan, 2000) for attachment anxiety and for attachment avoidance were used to generate four groups (e.g. Wang \& Scalise, 2010; Wei, Russell, Mallinckrodt, \& Vogel, 2007). According to Bartholomew and Phillip (1998), participants indicating low levels of attachment anxiety and avoidance were categorized as secure. Participants indicating low levels of attachment avoidance but high levels of attachment anxiety were considered preoccupied. Participants reporting low levels of attachment anxiety and high levels of attachment avoidance were considered dismissive. Participants indicating high levels of attachment anxiety and high levels of avoidance were categorized as fearful.

Based on participant scores in the anxiety and avoidance subscales of ECR-S, an additional independent variable was created for the new four attachment categories of participants. For example, participants who were in the low attachment anxiety and low attachment avoidance groups were categorized into the securely attached group. The same method was also applied to categorize participants into the preoccupied, dismissive, and fearful groups.

A multivariate analysis of variance (MANOVA) with a significance level $(\alpha)$ of .05 was conducted to determine whether participants report significant differences in QRI and CES-D scores (both dependent variables) across the four types of attachment styles. Participant scores of relationship quality (QRI) and depressive moods (CES-D) were analyzed through a 4 (secure, preoccupied, fearful, and dismissive attachment styles) X 2 (Taiwanese 
and Chinese vs. American) MANOVA with two dependent variables (quality of romantic relationships and depressive moods).

Hypothesis 5A. Levels of relationship quality will be statistically significantly different among Taiwanese/Chinese international students with different attachment categories.

Taiwanese/Chinese International Students. For the first analysis, only participants from Taiwan or China were selected. Then an ANOVA was conducted by entering QRI scores as the dependent variable and attachment style (secure, dismissive, preoccupied, and fearful) as the independent variable.

Hypothesis 5B. Levels of relationship quality will be statistically significantly different among American students with different attachment categories.

American Students. An ANOVA was conducted to determine whether American participants with different attachment styles reported statistically significantly different levels of QRI scores. Only participants identified as Americans were selected. Then an ANOVA was conducted by entering QRI scores as the dependent variable and attachment style (secure, dismissive, preoccupied, and fearful) as the independent variable.

Hypothesis 5C. Levels of depressive moods will be statistically significantly different among Taiwanese/Chinese international students with different attachment categories.

Taiwanese/Chinese International Students. First, only participants from Taiwan or China were selected. Then an ANOVA was conducted by entering CES-D scores as the dependent variable and four attachment styles (secure, dismissive, preoccupied, and fearful) as the independent variable. 
Hypothesis 5D. Levels of depressive moods will be statistically significantly different among American students with different attachment categories.

American Students. Only participants identified as Americans were selected. Then an ANOVA was conducted by entering CES-D scores as dependent variable and four attachment styles (secure, dismissive, preoccupied, and fearful) as the independent variable.

\section{Design}

A two-group comparison design (Macready, 2005) was used for this study. This design was selected because ethnicity cannot be manipulated and thus the comparison of the two ethnic groups (Americans and Taiwanese or Chinese) is appropriate to determine the differences between these two groups. Based on this design, the independent variables are ethnicity (Americans or Taiwanese/Chinese) based on participant self-identification and levels of insecure attachment (avoidant or anxious attachment styles) measured by the Experiences in Close Relationship Scale, short form (ECR-S; Wei, Russell, Mallinckrodt, \& Vogel, 2007). The dependent variables are levels of depressive moods and quality of romantic relationships, measured by the Center for Epidemiologic Studies Depression Scale (CES-D; Radloff, 1977) and The Quality of Relationships Inventory (QRI; Pierce, Sarason, \& Sarason, 1991). 


\section{CHAPTER THREE}

\section{RESULTS}

This chapter will first detail descriptive statistics, such as means, standard deviations, and sample frequencies. The second part of the chapter will present the hypotheses of the study followed by results of statistical analyses. Findings of two independent samples $t$-tests will be presented on levels of depressive moods (CES-D scores) and relationship quality (QRI scores) between Taiwanese or Chinese international student participants and American student participants with high levels of avoidant attachment. Findings of two more independent samples $t$-tests on levels of depressive moods or relationship quality between international student participants and American student participants with high levels of anxious attachment will also be presented. Furthermore, after attachment avoidance and anxiety scores were converted into four attachment categories (secure-low anxiety/low avoidance, preoccupiedhigh anxiety/low avoidance, dismissive-low anxiety/high avoidance, or fearful-high anxiety/high avoidance; Bartholomew \& Phillip, 1998), the finding of a MANOVA on the difference in the linear combination of two dependent variables (depressive moods and quality of romantic relationships) between participants with different attachment categories or ethnicity will be presented. Results will be presented after follow-up ANOVAs and multiple comparisons were conducted on levels of relationship quality between Taiwanese/Chinese international students with different attachment categories and American students with different attachment categories. The final section of quantitative results will include findings of follow-up ANOVAs and multiple comparisons after the MANOVA on levels of depressive moods between Taiwanese/Chinese international students with different attachment categories and then American students with different attachment categories, respectively. 
After quantitative results are presented, qualitative results from participant responses to the six open-ended questions about participant experiences in romantic relationships will be presented. The thematic analysis of Boyatzis (1998) on factors impacting romantic relationships, cultural effects, and taboos will be summarized. Then the integration of quantitative and qualitative findings will be discussed.

\section{Descriptive Statistics}

A total of 205 college students, 64 males (31.2\%) and 141 females (68.8\%) participated at locations in 16 U.S. states for this study. More participants identified as Americans $(n=139$, $67.8 \%)$ than Taiwanese or Chinese international students $(n=66,32.2 \%)$. Among American participants, most identified themselves as fourth generation or beyond $(n=95,46.1 \%)$, third generation $(n=27,13.1 \%)$, although a number of participants did not indicate any generation $(n$ $=62,30.1 \%)$. Most participants identified as Caucasian American $(n=126)$ and most international students who participated in this study identified as Chinese international students $(n=52,25.2 \%)$. See Table 1 for complete ethnicity information. Several student responses were excluded because of conflicting responses in the demographic answers, such as identifying as a Chinese international student but also identifying as a third generation American. 
Table 1

Ethnicity of Participants

\begin{tabular}{lrr}
\hline & Frequency & $\begin{array}{r}\text { Percentage of Total } \\
\text { Valid Responses }\end{array}$ \\
\hline African American & 2 & 1.0 \\
Asian American & 7 & 3.4 \\
Caucasian American & 126 & 61.2 \\
Hispanic American & 2 & 1.0 \\
Native American & 3 & 1.5 \\
Taiwanese international student & 11 & 5.3 \\
Chinese international student & 52 & 25.2 \\
Bi-or multi Ethnicity & 2 & 1.0 \\
Total & 205 & 100.0 \\
\hline
\end{tabular}

Most participants identified themselves as undergraduate $(n=103,50.2 \%)$ students. A number of participants were enrolled in masters $(n=57,27.8 \%)$, doctorate $(n=41,20.0 \%)$, or professional degree $(n=5.2 .4 \%)$ programs. A majority of students identified themselves as heterosexual ( $n=168,80.2 \%)$ and a minority of participants identified themselves as homosexual ( $n=6,2.9 \%)$, lesbians $(n=4,2.0 \%)$, questioning (individuals who are questioning their sexual identity or do not identify as either heterosexual, lesbian, gay, bisexual, or transgender, $n=3,1.5 \%$ ), or with double orientations (bisexual $=8,3.9 \%$; bisexual and heterosexual, $n=1, .5 \%$; lesbian and bisexual, $n=1, .5 \%)$, while 15 participants $(7.3 \%)$ did not disclose any sexual orientation. Most participants indicated their relationship status to be dating and committed $(n=114,55.6 \%)$, married $(n=35,17.1 \%)$, cohabiting $(n=36,17.6 \%)$, or dating and not committed $(n=21,10.2 \%)$. Additionally, a majority of participants $(n=184,89.8 \%)$ indicated no children in the household and a small number of participants $(n=22,10.7 \%)$ reported having one to four children residing in their households (Table 2). In terms of 
employment status, most participants indicated working part-time $(n=75,36.6 \%)$ while a similar number of participants reported being unemployed $(n=70,34.1 \%$; Table 3$)$.

Table 2

Number of Children in Residence

Frequency Percentage

\begin{tabular}{lrr}
0 & 184 & 89.3 \\
1 & 8 & 3.9 \\
2 & 7 & 3.4 \\
3 & 3 & 1.5 \\
4 & 4 & 1.9 \\
Total & 206 & 100.0 \\
\hline
\end{tabular}

Table 3

Employment

Frequency

Percentage

Full-time

60

29.1

Part-time

75

36.6

Unemployed

70

34.0

205

29.1

The mean of all responses from both Taiwanese/Chinese international students and American students were calculated on the anxiety subscale and on the avoidance subscale of the ECR-S. Participants were categorized into high or low attachment anxiety groups based on their responses relative to the calculated mean. The high attachment anxiety group encompassed participants who reported scores higher than the average of all participant responses in the attachment anxiety subscale of ECR-S in this dissertation study. The low attachment anxiety group consisted of participants who reported scores lower than the average of all participant responses in the attachment anxiety subscale of ECR-S. Consistently, the high attachment 
avoidance group included those with scores higher than the average of participants in the attachment avoidance subscale of ECR-S while the low attachment anxiety group encompassed participants who reported scores lower than the average of all participants in the attachment anxiety subscale of ECR-S (Table 4).

Table 4

Means, Standard Deviations, and Percentage of Anxious and Avoidant Participants in Chinese/Taiwanese International Students and American Students

\begin{tabular}{|c|c|c|c|}
\hline \multirow{2}{*}{$\begin{array}{l}\text { Insecure Attachment } \\
\text { Anxious Attachment }\end{array}$} & \multirow[b]{2}{*}{ Low } & Taiwanese/Chinese & Americans \\
\hline & & \multicolumn{2}{|c|}{$M_{\text {total }}=14.84, S D=2.55$} \\
\hline & & $\begin{array}{l}n=35(53 \% \text { among } \\
\text { international students })\end{array}$ & $\begin{array}{l}n=59(42 \% \text { among } \\
\text { American students) }\end{array}$ \\
\hline & High & & \\
\hline & & $\begin{array}{l}n=31 \text { (47\% among } \\
\text { international students) }\end{array}$ & $\begin{array}{l}n=80 \text { (58 \% among } \\
\text { American students) }\end{array}$ \\
\hline \multirow[t]{4}{*}{ Avoidant Attachment } & Low & \multicolumn{2}{|c|}{$\mathrm{M}_{\text {total }}=12.28, \mathrm{SD}=4.39$} \\
\hline & & $\begin{array}{l}n=31 \text { ( } 47 \% \text { among } \\
\text { international students) }\end{array}$ & $\begin{array}{l}n=99(71 \% \text { among } \\
\text { American students })\end{array}$ \\
\hline & High & & \\
\hline & & $\begin{array}{l}n=35 \text { (53\% among } \\
\text { international students) }\end{array}$ & $\begin{array}{l}n=40(29 \% \text { among } \\
\text { American students })\end{array}$ \\
\hline
\end{tabular}

Among Taiwanese or Chinese international students, 31 (47\%) international students can be categorized as highly anxiously attached. Among American students, 80 individuals (58\%) reported high levels of attachment anxiety. Thus, a larger number of responses from American students $(n=80)$ than Taiwanese or Chinese international students $(n=31)$ with attachment anxiety were used in the independent samples $t$-tests for attachment anxiety (Table 5) while similar numbers of responses from Taiwanese or Chinese international students $(n=35)$ and American students $(n=40)$ with high levels of attachment avoidance were used in the independent samples $t$-tests for attachment avoidance (Table 6). Higher percentage of 
international students ( $n=35,53 \%$ among international students) indicated higher avoidant attachment than American students ( $n=40,29 \%$ among American students).

Table 5

Means, Standard Deviations of Depressive Moods, Relationship Quality of Participants with High Attachment Anxiety

\begin{tabular}{lcccc}
\hline & $\begin{array}{r}\text { Ethnicity or Home } \\
\text { Country }\end{array}$ & $n$ & Mean & Std. Deviation \\
\hline $\begin{array}{l}\text { CESD (levels of } \\
\text { depressive moods) }\end{array}$ & $\begin{array}{r}\text { Taiwanese or Chinese } \\
\text { international students } \\
\text { American students }\end{array}$ & 31 & 38.58 & 9.74 \\
\hline QRI scores (quality of & Taiwanese or Chinese & 31 & 72.06 & 10.90 \\
romantic relationships) & $\begin{array}{r}\text { international students } \\
\text { American students }\end{array}$ & 80 & 81.00 & 12.55 \\
\hline
\end{tabular}

Table 6

Means, Standard Deviations of Depressive Moods, Relationship Quality of Participants with High Attachment Avoidance

\begin{tabular}{|c|c|c|c|c|}
\hline & $\begin{array}{r}\text { Ethnicity or Home } \\
\text { Country }\end{array}$ & $n$ & Mean & Std. Deviation \\
\hline \multirow{2}{*}{$\begin{array}{l}\text { CESD (levels of } \\
\text { depressive moods) }\end{array}$} & $\begin{array}{l}\text { Taiwanese or Chinese } \\
\text { international students }\end{array}$ & 35 & 40.83 & 10.27 \\
\hline & American students & 40 & 41.70 & 6.55 \\
\hline \multirow{2}{*}{$\begin{array}{l}\text { QRI scores (quality of } \\
\text { romantic relationships) }\end{array}$} & $\begin{array}{l}\text { Taiwanese or Chinese } \\
\text { international students }\end{array}$ & 35 & 70.97 & 10.03 \\
\hline & American students & 40 & 79.55 & 15.41 \\
\hline
\end{tabular}

\section{Preliminary Analysis}

Prior to conducting statistical analyses, data from the Quality of Relationships Inventory (QRI), Experiences in Close Relationship Scale (ECR-S), and the Center for Epidemiologic Studies Depression Scale (CES-D) were examined for their fitness with multivariate assumptions. Participant responses that omitted any question in the QRI, ECR-S, and CES-D were eliminated. 
Hypothesis 1. Levels of depressive symptoms will be significantly different between American college students and Taiwanese/Chinese international students with high levels of attachment avoidance.

The select cases function on SPSS was used to select only participants with high levels of attachment avoidance for this analysis. An independent samples $t$-test was conducted to determine if there was a significant difference in levels of depressive moods (CES-D scores) between Taiwanese/Chinese international students and American students with high levels of attachment avoidance (scores of the attachment avoidance subscale in ECR-S). There was a significant effect detected in the Levene's test that indicated unequal variances between the Taiwanese/Chinese international students and American students groups. Thus, the $t$-test of equal variance not assumed was interpreted. The result $(t=-.43, p=.668)$ indicated there was no statistically significant difference in the levels of depressive moods between international students and American students. Taiwanese/Chinese international students with attachment avoidance $(n=35, M=40.83)$ and American students with attachment avoidance $(n=40, M=$ 41.7) reported similar levels of CES-D scores. This hypothesis was not supported by the finding.

Hypothesis 2. Levels of relationship quality will be significantly different between American college students with high levels of attachment avoidance and Taiwanese/Chinese international students with high levels of attachment avoidance.

The select cases function on SPSS was used to select only participants with high levels of attachment avoidance for this analysis. There was a significant difference in the Levene's test, and thus the $t$-test of equal variance not assumed was interpreted. A statistically significant difference $(t=-2.89, p=.005)$ in levels of QRI scores between international 
students with high levels of attachment avoidance and American students with high levels of attachment avoidance was found. Taiwanese/Chinese international students $(M=70.97)$ reported significantly lower QRI scores than American students $(M=79.55)$. The hypothesis was supported by the significant difference of relationship quality detected between Taiwanese/Chinese international students with high attachment avoidance and American students with high attachment avoidance.

Hypothesis 3. Levels of depressive moods will be significantly different between American college students and Taiwanese/Chinese international students with high levels of attachment anxiety.

The select cases function on SPSS was used to select only participants with high levels of attachment anxiety for this analysis. An independent samples $t$-test was conducted to determine the significance of levels of depressive moods between Taiwanese/Chinese international students and American students with high levels of attachment anxiety. There was a significant effect detected in the Levene's test which indicated unequal variances between the Taiwanese/Chinese international students and American student groups. Thus, the $t$-test of equal variance not assumed was interpreted. The result $(t=.27, p=.786)$ indicated there was no statistically significant difference in the levels of CES-D scores between the international students and American students. Anxiously attached Taiwanese/Chinese international students $(n=31, M=38.58)$ and anxiously attached American students $(n=80, M=38.08)$ reported similar levels of depressive moods. 
Hypothesis 4. Levels of relationship quality will be significantly different between American college students and Taiwanese/Chinese international students with high levels of attachment anxiety.

The select cases function on SPSS was used to select only participants with high levels of attachment anxiety for this analysis. In terms of the quality of romantic relationships, no significant difference in variance was detected in the Levene's test, and thus the $t$-test of equal variance assumed was interpreted. A statistically significant difference $(t=-3.49, p=.002)$ in levels of QRI scores between international students and American students was found. Anxiously attached Taiwanese/Chinese international students $(n=31, M=72.06)$ reported significantly lower QRI scores than anxiously attached American students $(n=80, M=81.00)$. The hypothesis was supported by the significant difference of relationship quality detected between anxiously attachment Taiwanese/Chinese international students and American students.

\section{Evaluations of Assumptions for MANOVA}

Before testing the main hypothesis with a MANOVA, assumptions of sample representativeness, sample size, levels of measurement, normality of the distribution, homogeneity of variances, linearity relationship between dependent variables, homogeneity of variance-covariance matrices, and multi-collinearity must be evaluated (Abu-Bader, 2010). The results of tests for these assumptions will be discussed in the following paragraphs.

Tests of representativeness, sample size, normality, and levels of measurement. For the research sample, 205 participants were recruited from 30 randomly selected universities which were listed as highly research intensive universities on the website of Carnegie Classification of Institutions of Higher Education (Carnegie Foundation of Excellence Teaching, 
2010), and thus the representativeness of the sample appears to have been satisfied. The number of participants is larger than the minimum of 30 participants per group required for MANOVA, and thus this assumption was met.

Furthermore, normality of sums of the two dependent variables, QRI and CES-D were examined. Sums of participant responses to the QRI and CES-D were normally distributed in the Levene's test of equality of error variances (QRI: $p=.06>.05$; CES-D: $p=.18>.05$ ). Using SPSS to test skewness and kurtosis of the two dependent variables, the QRI scores were negatively skewed but presented no significant kurtosis $($ Table 7 ; skewness coefficient $=-$ $0.65 / 0.17=-3.82<-1.96$ and kurtosis coefficient $=-.26 / .34=-0.77>-1.96)$. The significance of skewness or kurtosis was determined when the coefficient values fell outside the range between -1.96 and 1.96 (Abu-Bader, 2010).

Table 7

Skewness and Kurtosis of Dependent Variables

\begin{tabular}{crrrrrr}
\hline & \multicolumn{4}{c}{ Skewness } & \multicolumn{2}{c}{ Kurtosis } \\
& Statistic & Std. & Skewness & Statistic & Std. & Kurtosis \\
& Error & Coefficient & & Error & Coefficient \\
QRI & -0.65 & 0.17 & -3.82 & -0.26 & 0.34 & -0.77 \\
CES-D & 0.55 & 0.17 & 3.22 & 1.40 & 0.34 & 3.22 \\
\hline
\end{tabular}

Normality of the dependent variables (levels of depressive moods and quality of romantic relationships) across high or low levels of anxious or avoidant attachment scores was tested with the Kolmogorov-Smirnov test by measuring the skewness and kurtosis for dependent variables. Violation of normality of CES-D scores was found $(d f=205, p<.001)$. The assumption of normality of relationship quality scores was also found $(d f=205, p<.001)$. The distribution of the sum of relationship quality scores was positively skewed across all levels of anxious attachment. Similarly, QRI scores were not normally distributed in both 
high and low (relative to $\mathrm{M}=12.17, d f=76,130$, kurtosis coefficient $=-1.89$, skewness coefficient $=.75)$ attachment avoidance scores.

When the sum of anxious attachment scores were above the mean of all responses in the anxious scale $(\mathrm{M}=12.17)$, violation of normality was found (above: $d f=95, p<.001$; below: $d f=111, p<.001)$. The distribution of the sum of QRI was positively skewed across all levels of anxious attachment. QRI scores were not normally distributed in both groups of high and low attachment avoidance (relative to $\mathrm{M}=12.17, d f=76,130$, respectively). Despite the non-normality found, it was not sufficient to cause concern given that the skewness and kurtosis of relationship quality or depressive moods were not beyond the range between $+/-5$.

According to Abu-Bader (2010), independent variables should be categorical while dependent variables should be interval. Thus, non-categorical independent variables, anxious and avoidant attachment scores were transformed into categorical data before the MANOVA test. More specifically, scores higher than the mean of attachment anxiety scores were transformed into two (high level of anxiety) and those lower than the mean attachment anxiety were transformed into one (low level of anxiety). The same method was used to transform the attachment avoidance scores. Because interval data was collected for the dependent variables (QRI and CES-D), these scores remained the same. 
Tests of the homogeneity of variances. Homogeneity of variances was evaluated with a Levene's test of error variances. Significant results in QRI and CES-D scores were obtained ( $p$ $<.001)$. Because Levene's test of equality of error variance was violated, a more conservative test, Wilks' Lambda, was applied to the MANOVA test (Titsworth, Hunt, \& Allen, 2009).

Linearity relationship between dependent variables. To assess the linearity between dependent variables, Bartlett's Test of Sphericity was conducted. A significant result was determined $(p<.001)$, indicating a significant correlation between the two dependent variables, levels of relationship quality and depressive moods. Therefore, the required assumption of linearity between dependent variables for MANOVA was met (Abu-Bader, 2010).

Homogeneity of variance-covariance matrices. The Box test of Equality of Covariance Matrices was applied to assess the homogeneity in the variance and covariance matrices. A significant result $(p<.001)$ was detected, which violated this assumption. Additionally, homogeneity of variances was evaluated in Levene's test of error variances. A significant result was obtained $(p<.001)$. Box's test of equality of covariance matrices was used to assess the homogeneity of variance-covariance matrices. A significant result was obtained, and thus an ANOVA should be considered. Multicollinearity was examined by the Residual SSCP Matrix, and the correlation between the two dependent variables, QRI and CES-D was smaller than .80 $(r=.01)$. Thus, this assumption was met. After examinations of assumptions, a MANOVA was conducted to investigate the research hypotheses of this dissertation study. These findings will be discussed in the following section.

\section{Examination of Hypotheses}

Hypothesis 5. The linear combination of two dependent variables (levels of relationship quality and depressive moods) will be significantly different between American college students 
and Taiwanese/Chinese international students with different attachment categories (secure, preoccupied, dismissive, or fearful; Bartholomew \& Phillip, 1998).

Based on the same method of previous studies, responses on the Experiences in Close Relationship Scale (ECR; Fraley, Waller, \& Brennan, 2000) for attachment anxiety and for attachment avoidance were used to generate four groups (e.g. Wang \& Scalise, 2010; Wei, Russell, Mallinckrodt, \& Vogel, 2007). According to Bartholomew and Phillip (1998), participants indicating low levels of attachment anxiety and avoidance were categorized as secure. Participants indicating low levels of attachment avoidance but high levels of attachment anxiety were considered preoccupied. Participants reporting low levels of attachment anxiety and high levels of attachment avoidance were considered dismissive. Participants indicating high levels of attachment anxiety and high levels of avoidance were categorized as fearful. Based on participant scores in the anxiety and avoidance subscales of ECR-S, an additional independent variable was created for the new four attachment categories of participants. For example, participants who were in the low attachment anxiety and low attachment avoidance groups were categorized into the securely attached group. The same method was also applied to categorize participants into the preoccupied, dismissive, and fearful groups.

A multivariate analysis of variance (MANOVA) with a significance level $(\alpha)$ of .05 was conducted to determine whether participants report significant differences in QRI and CES-D scores (both dependent variables) across the four types of attachment styles. Participant scores of relationship quality (QRI) and depressive moods (CES-D) were analyzed through a 4 (secure, preoccupied, fearful, and dismissive attachment styles) X 2 (Taiwanese 
and Chinese vs. American) MANOVA with two dependent variables (quality of romantic relationships and depressive moods).

\section{Interaction and Main Effects}

Attachment group (that is, secure, preoccupied, dismissive, or fearful) and ethnicity (Taiwanese/Chinese or American) were entered as independent variables and the two variables of depressive moods and quality of romantic relationships were entered as the dependent variables in the MANOVA. In the MANOVA results, there was no statistically significant interaction between attachment styles and ethnicity $(F=.63, p=.705$, observed power $=.25$, Table 8). Thus, the main effects of attachment styles and ethnicity or home country were evaluated. A statistically significant difference on the linear combination of CES-D and QRI was found among the attachment styles $(F=7.48, p<.001$, partial eta squared $=.10$, observed power $=1.00$ ). A statistically significant difference on the linear combination of CES-D and QRI also was found between American students and Taiwanese or Chinese international students $(F=11.59, p<.001$, partial eta squared $=.11$, observed power $=.99)$.

Table 8

MANOVA on Relationship Quality and Depressive moods between American and Taiwanese/Chinese

\begin{tabular}{rrrr}
\hline & $F$ & $p$ & Power \\
\hline Attachment style & 7.48 & $.000^{*}$ & 1.00 \\
Ethnicity & 11.59 & $.000^{*}$ & .99 \\
Attachment Style* Ethnicity & .63 & .705 & .25 \\
\hline
\end{tabular}

$* p<.001$

Given that a significant effect of attachment styles was found, the tests of betweensubjects effects on relationship quality and depressive moods were interpreted. A statistically significant effect was detected in the levels of QRI scores among the four attachment styles ( $F$ 
$=6.30, p<.001$, partial eta squared $=.09$, observed power $=.96$, Table 9 ).

Taiwanese/Chinese international students $(M=74.14)$ indicated significantly lower levels of relationship quality than American students $(\mathrm{M}=82.31)$. Consistently, participants with different attachment styles indicated significantly different levels of CES-D scores $(F=8.39$, $p<.001$, partial eta squared $=.11$, observed power $=.99)$. Taiwanese/Chinese international students $(M=38.88)$ indicated significantly lower levels of depressive moods than American students $(M=39.51)$.

Table 9

ANOVA on Relationship Quality and Depressive moods between American and Taiwanese/Chinese

\begin{tabular}{|c|c|c|c|}
\hline & $F$ & $p$ & Power \\
\hline \multicolumn{4}{|l|}{ QRI } \\
\hline Ethnicity & 23.21 & $.000 *$ & 1.00 \\
\hline Attachment styles & 6.3 & $.000 *$ & .96 \\
\hline Attachment Style* Ethnicity & .65 & .582 & .19 \\
\hline \multicolumn{4}{|l|}{ CES-D } \\
\hline Ethnicity & .45 & .505 & .10 \\
\hline Attachment styles & 8.39 & $.000 *$ & .99 \\
\hline Attachment Style* Ethnicity & .71 & .547 & .20 \\
\hline
\end{tabular}

$* p<.001$

In the pair-wise post hoc tests among the four attachment categories, a statistically significant difference was found in levels of relationship quality between participants with secure $(\mathrm{M}=82.92)$ and fearful $(\mathrm{M}=73.00)$ attachment style $(p<.001)$. A statistically significant difference was found in levels of relationship quality between participants with preoccupied $(\mathrm{M}=82.10)$ and fearful $(\mathrm{M}=73.00)$ attachment style $(p<.001)$. All of other pairs of attachment categories were non-significant. 


\section{QRI Scores}

After a statistically significant main effect was found in the MANOVA, follow-up ANOVAs were conducted to determine whether participants with different attachment styles reported significantly different levels of relationship quality between the American student group and Taiwanese or Chinese international student group. Means and standard errors of QRI scores reported by Taiwanese or Chinese international and American students are presented in Table 10.

Table 10

Relationship Quality, Four Attachment Style, and Ethnicity or Home Country

\begin{tabular}{cllr}
\hline Ethnicity or Home Country & $\begin{array}{l}\text { Attachment } \\
\text { Styles }\end{array}$ & Mean & Std. Error \\
\hline Taiwanese or Chinese & Secure & 76.06 & 2.50 \\
international students & Dismissive & 74.56 & 2.52 \\
& Preoccupied & 78.75 & 3.08 \\
& Fearful & 67.18 & 2.59 \\
\hline \multirow{2}{*}{ American students } & Secure & 85.57 & 1.61 \\
& Dismissive & 83.87 & 2.76 \\
& Preoccupied & 82.84 & 1.44 \\
& Fearful & 76.96 & 2.14 \\
\hline
\end{tabular}

Hypothesis 5A. Levels of relationship quality will be significantly different between Taiwanese/Chinese international students with different attachment categories.

Taiwanese/Chinese International Students. For the first analysis, only participants from Taiwan or China were selected. Then an ANOVA was conducted by entering QRI scores as the dependent variable and attachment style (secure, dismissive, preoccupied, and fearful) as the independent variable. 
A statistically significant result was detected in the QRI scores of Taiwanese or Chinese international students across the four attachment styles $(F=5.05, p=.003$, partial eta squared $=.20$, observed power $=.90$ ). Thus, post-hoc independent samples $t$-tests with Bonferroni corrections were conducted to assess significant differences in levels of relationship quality between the two attachment styles. A statistically significant difference was found in QRI scores between participants with secure $(M=76.06)$ and fearful $(M=67.18)$ attachment style $(p=.024)$. Taiwanese/Chinese participants with secure attachment indicated significantly higher levels of relationship quality than those with fearful attachment style. Another significant difference was also detected between QRI scores between Taiwanese/Chinese participants with pre-occupied $(\mathrm{M}=78.75)$ and fearful attachment $(\mathrm{M}=$ 67.18) styles $(p=.005)$. Taiwanese/Chinese participants with preoccupied attachment style indicated significantly higher levels of relationship quality than participants with fearful attachment style. Interestingly, Taiwanese/Chinese participants with secure and preoccupied attachment styles indicated similar levels of relationship quality $(p=1.000)$.

Hypothesis 5B. Levels of relationship quality will be significantly different between American students with different attachment categories.

American Students. An ANOVA was conducted to determine whether American participants with different attachment styles reported significantly different levels of QRI scores. Only participants identified as Americans were selected. Then an ANOVA was conducted by entering QRI scores as the dependent variable and attachment style (secure, dismissive, preoccupied, and fearful) as the independent variable. A statistically significant result was detected among American students with the four attachment categories $(F=3.07, p$ $=.030$, partial eta squared $=.06$, observed power $=.71)$. Thus, post-hoc independent samples 
$t$-tests with Bonferroni corrections were conducted to determine significant differences in levels of relationship quality between each two attachment styles. One statistically significant difference $(p=. \underline{019})$ was determined between QRI scores of American participants with secure $(\mathrm{M}=85.57)$ and fearful $(\mathrm{M}=76.96)$ attachment styles. American participants with secure attachment indicated significantly higher levels of relationship quality than those with fearful attachment.

\section{CES-D Scores}

Because a significant effect was found in the MANOVA, follow-up ANOVAs were conducted to determine whether participants with different attachment styles reported significantly different levels of depressive moods for each ethnicity group. Means and standard errors of CES-D scores reported by Taiwanese or Chinese international and American students are presented in Table 11.

Table 11

Depressive Moods, Four Attachment Style, and Ethnicity or Home Country

\begin{tabular}{cllr}
\hline Ethnicity or Home Country & Attachment & Mean & Std. Error \\
& Styles & & \\
\hline Taiwanese or Chinese & Secure & 35.71 & 1.45 \\
international students & Dismissive & 42.67 & 1.41 \\
& Preoccupied & 38.25 & 1.73 \\
& Fearful & 38.88 & 1.45 \\
\hline \multirow{2}{*}{ American students } & Secure & 37.34 & .90 \\
& Dismissive & 43.00 & 1.54 \\
& Preoccupied & 36.78 & .814 \\
& Fearful & 40.92 & 1.20 \\
\hline
\end{tabular}


Hypothesis 5C. Levels of depressive moods will be significantly different between Taiwanese/Chinese international students with different attachment categories.

Taiwanese/Chinese International Students. First, only participants from Taiwan or China were selected. Then an ANOVA was conducted by entering CES-D scores as the dependent variable and four attachment styles (secure, dismissive, preoccupied, and fearful) as the independent variable. No statistically significant result was detected among Taiwanese or Chinese international students with the four attachment styles $(F=2.29, p=.088$, observed power $=.55)$. Participants from Taiwan or China with the four attachment styles indicated similar levels of depressive moods $\left(M_{\text {secure }}=35.71, M_{\text {dismissive }}=42.67, M_{\text {preoccupied }}=38.25\right.$, $\left.M_{\text {fearful }}=38.88\right)$.

Hypothesis 5D. Levels of depressive moods will be significantly different between American students with different attachment categories.

American Students. Only participants identified as Americans were selected. Then an ANOVA was conducted by entering CES-D scores as dependent variable and four attachment styles (secure, dismissive, preoccupied, and fearful) as the independent variable. A statistically significant result was detected among American students who reported the four attachment styles $(F=9.47, p<.001$, partial eta squared $=.17$, observed power $=1.00)$. Thus, post-hoc independent samples $t$-tests with Bonferroni corrections were conducted to assess significant differences in levels of CES-D scores between every two attachment styles. A statistically significant difference in CES-D scores was determined between participants with secure $(M=37.34)$ and fearful $(M=40.92)$ attachment styles $(p=.023)$. Participants with secure attachment indicated significantly lower levels of depressive moods than participants with fearful attachment. Participants with dismissive $(M=43.00)$ and preoccupied $(M=$ 
36.78) attachment styles also reported significantly different levels of depressive moods ( $p$ $<.001)$. Dismissive individuals indicated significantly higher levels of depressive moods than preoccupied individuals. Fearful $(\mathrm{M}=40.92)$ American participants also indicated significantly higher levels of depressive moods than preoccupied $(M=36.78)$ American participants $(p=.003)$.

\section{Qualitative Analysis: Factors Impacting Romantic Relationships}

In response to the first open-ended question, 180 participants identified factors that impact relationship quality with the romantic partner. On the individual level, more situational factors (stress or mood of self or partner) as well as stable factors, positive characteristics of partner were identified as significant factors impacting the relationship quality (maturity, patience, loyalty, honesty, genuineness, humor, optimistic, ambitious, and responsible). On the relationship level, the similarities and compatibility of both partners were identified in addition to factors associated with interactions within the relationship. Similarity and compatibility of both intrinsic (e.g., interests, values, religion, morals, beliefs, attractiveness, education level, willingness to travel) and extrinsic factors (e.g., age and education levels) were stressed. Identified relationship factors included respect, appreciation, spending time together, levels of commitment, planning a future together, communication, connection/emotional intimacy, sex, trust, gender roles, extramarital relationships, openness, forgiveness, and leaving space.

On the systemic level, factors related to family of origin, relationship and cultural background were highlighted by participants (divorced parents, familial disapproval of partner, SES difference, parenting, cultural differences, heterosexism, language barriers, immigration status, physical distance, social support from family and friends, past romantic relationships, and communication). From a broader perspective, environmental factors were also pointed out 
(schedule, money, employment/work, education, childcare, health, and housing: sharing a house with many other people). While many participants indicated that time spent together, distance, pressure from families of origin, moods, stress, and gender roles significantly impacted relationship quality, participants also emphasized that communication, trust, solving problems together, negotiation, and understanding and dealing with emotional connection can also be mediating factors for relationship quality.

\section{Cultural Effects and Differences in Romantic Relationships}

Compatibility and trust/honesty were both among the top three most frequently identified factors by both the American participants and Taiwanese/Chinese participants. Many participants indicated that compatibility of interests, values, intelligence, religion, culture, age, and levels of maturity impact their relationship quality significantly. Distance was also the fifth and the third most frequently identified in the American and Taiwanese/Chinese group, respectively. However, cultural differences between the American and Taiwanese/Chinese groups were observed among the identified factors which impacted relationship quality. More

specifically, communication was the most frequently identified factor among American participants but one of the least frequently identified factors among Taiwanese/Chinese participants. Similarly, sex and/or physical and emotional attraction was the fourth most frequently identified factor in the American group but the least frequently identified factor in the Taiwanese/Chinese group. Furthermore, Taiwanese/Chinese participants emphasized family responsibility (care-giving to parents, helping or financial support for family of origin) more frequently than American participants as it was the third most frequently identified but rarely identified by American participants. Additionally, certain Taiwanese/Chinese participants 
identified "understood by partner," which was considered a deep understanding and acceptance of a person as a significant factor but none of the American participants reported this factor.

Regarding cultural factors, a number of Caucasian American participants stated that they experience no cultural difference in their romantic relationships. Similarly, participants who were involved in a romantic relationship with a same-race, same culture, or same country of origin partner stated that cultural factors did not impact their relationship. For example, one participant wrote "We are of a similar culture, so we can understand the significance behind holidays and can understand implications behind difference phrases and sayings." In contrast, some participants indicated that regional cultural differences affected their relationships, such as "He is 'East Coast' and was raised not to express emotion and keep things inside. I was raised 'West Coast' from a family dynamic of sharing and expressing feelings.'

A larger number of American participants than Taiwanese/Chinese participants stated no impact of their cultural backgrounds or the American culture because both partners are from the same culture. Among Taiwanese/Chinese participants, certain participants indicated no influence of their cultural backgrounds but most participants identified that similar cultural background resulted in similar values and traditions. A higher proportion of Taiwanese/Chinese participants reported current engagement in cross-cultural or interracial relationships and expressed appreciation, curiosity, and acceptance of each other. For instance, one participant indicated that "As an interracial couple, we learn about each other's cultures," and another participant reported "I value family relationships and appreciate his family's (and extended family) acceptance and welcoming me into the family, even though we never public expressed our affection and none of his family members explicitly acknowledged our relationship." 
Both in the American and Taiwanese/Chinese groups, most participants who engaged in cross-cultural and cross-racial romantic relationships expressed the significance of genuine curiosity, understanding, acceptance, respect for partner's culture, willingness to learn new cultures, and the effort of "putting things into perspective" resulted in positive relationship quality. For instance, one participant described the adjustment of different cultures, "We both love learning about new cultures while still taking pride in our own." While differences were not always described as having a negative impact, certain participants indicated that cultural values manifested in gender roles which occasionally resulted in discomfort or disagreement, such as the man pays the bill, or the woman takes care of the husband and children.

Several participants also reported negative experiences about negative reactions of others or family members toward cross-racial relationships, such as making racist comments or expressing disapproval, such as "sometimes my family members or his family will say something that annoys us about him being black or me being white." The impact of the American culture was mostly described negatively by American participants. For example, participants stated that "Divorce is a big issue in American culture," "Sex was pushed very early," "The contradictory messages that Americans receive about feminine physical ideals make me more self-conscious and sometimes affect my confidence and self-esteem in the relationship," or "Maybe it gives me (or "us" as Americans) too high of standards for relationships. Because of the media we expect to have passionate love for the rest of our lives, when in reality what we need and should be looking for is companionate love."

Participants described the impact of the American culture on characteristics, such as becoming more independent. Furthermore, the cultural differences between heterosexual and same-sex relationships were also reported by participants. One participants indicated that "It 
affect his moods when I don't do what he wants me to do. Gay relationships are based on who has the power, and he wants it all the time. Homosexuality isn't the most popular thing and we can't really have public displays of affection without worry of danger to our persons."

In addition to the "no impact" response from many American participants, a smaller number of American participants indicated neutral or positive impact of the American culture on their romantic relationships. For example, one participants engaged in a cross-cultural relationship reported that "The American culture doesn't impact our relationship, but it does make me grateful to be married to such a feminist." Other participants also expressed positive influences of the American culture, such as "The American culture makes us not have to consult parents, siblings, friends on dating," "It actually taught me what not to do in a relationship but go back to my culture and see what worked for my parents," "I think that American culture is very accepting of our relationship (I am a female Asian-American, he is a male CaucasianAmerican)," "Living in America provides us with the freedom to live how we want together and determine what we want our relationship to be," and "American culture made us more independent from each other, even economically."

A higher percentage of American participants reported negative impact of the American culture on romantic relationships than Taiwanese/Chinese participants. For example, "I am not thin like the American culture says I have to be. My husband loves me but is always wanting me to lose weight," "American culture is confusing. It has the picture perfect family ideals that women strive for, and then there are shows like Jersey Shore that make men, and some women, try to just hook up with whoever they can," "The vulgarity of certain visual stimuli that are deemed acceptable, which have put several road bumps per say," "Especially how casual dating is portrayed, with people breaking up, cheating, divorcing, and lying to their partner," "American 
culture has influenced my relationship in that the theory or idea that long distance relationships have a high failure rate has brought a level of unwarranted doubt into if continuing my long distance relationship is worth it," or "We both come from conservative families, which is difficult to find someone of similar viewpoints in the American culture of today. Materialism influences gifts that are exchanged, instead of focusing on needs other than physical needs."

In contrast, a higher percentage of Taiwanese/Chinese participants acknowledged impact of their cultural backgrounds or the American culture on romantic relationships. More specifically, most participants identified the impact to be preferences of similarities, the choice of a partner, and cultural norms in romantic relationships. Certain male participants expressed preferences of a partner from the same or similar (Chinese) culture and other participants described cultural expectations in romantic relationships, such as "similar backgrounds makes us have many similarities," "I am not reject people from another countries, but Chinese maybe the primary choice for a relationship," "To Chinese men, a woman who prefers to make a lot of efforts to her husband and children as well as related family affairs is good," or "Dating is for marriage, not just for fun. Dating is when you are seeing someone exclusively, not seeing multiple people at the same time."

A higher rate of the Taiwanese/Chinese participants identified positive impact of the American culture. Identified cultural factors were the openness in expression of affection, acceptance of same-sex relationship, and supporting each other while learning and adjusting to the American culture, such as "There is a peer learner aspect to our relationship, which can turn into mutual support," "I think that the American culture makes my partner more open in relationship, especially on the sexuality," "To be more acceptable for diversity in the society," "I value the feeling of love more than before. I begin to think about homosexual and one night 
stand," "I have become more open-minded and accepting of changes and differences," or "I think American culture such as tolerance and open will impact your current romantic relationship because partners can tolerate each other so that they can go well with each other in the life."

A small number of Taiwanese/Chinese participants reported negative impact of the American culture on their romantic relationships. Reported impact included the AmericanChinese cultural conflicts in relationships or adverse attitude toward the American sexual attitude perceived by participants, such as "I am very open to American culture and I like American culture. Whenever there is a conflict between my American-affected cultural beliefs with my traditional Chinese cultural beliefs, I feel so powerless and struggle," "It is nothing here to date someone or have sex with something, since it means nothing here."

\section{Taboos and Interactions Impacts on Relationship}

The most common taboos reported by both American and Taiwanese/Chinese participants were discussions about previous relationships, betrayals, or lies. The second most common taboos were insulting, cursing, degrading partner or yelling. Certain participants identified divorce, partner's family, deployment in the military, attractiveness of the opposite sex, masturbating, or sex as taboos. Religion or politics were also indicated as taboos by a majority of American participants. Similarly, a majority of Taiwanese/Chinese participants identified taboos as disrespect, betrayals, lying, shouting, making personal attacks (defects), or politics. No significant difference was determined about taboos between the American and Taiwanese/Chinese groups.

While a number of taboos were identified, a majority of American participants emphasized the significant impact of spending time together, communication, and negotiation with partners on relationship quality and moods. Most participants highlighted the close 
relationship between positive moods, good communication and relationship quality. Similarly, a number of participants indicated the strong relationship between moods and positive interactions, such as "I like to make sure that we're both happy and communicative. My interactions with my partner make the relationship quality more positive, and the effects of interaction on my mood really depend on the type of interaction." Some participants further described how a lack of responses, emotional distance, and arguments negatively influenced the relationship quality. One participant reported that he tends to feel anxious, upset, and vulnerable when communication issues occurred, such as a partner ceasing to respond to text messages. For example, partner emotional withdrawal elicited individual attachment anxiety. "Partner tends to withdraw when he is upset, which would make me wonder if I did something wrong or I feel the need to help him feel better."

\section{Integration of Quantitative and Qualitative Data}

Qualitative participant responses confirmed and described the close relationship between moods and relationship quality as well as the negative impact of emotional or physical distance, and lack of communication (attachment avoidance) on relationship quality and depressive moods. Consistently, participants also confirmed the association between attachment anxiety and relationship quality by describing elevated levels of anxiety after partner emotional withdrawal. Indirectly, communication, trust, solving problems together, negotiation, and understanding and dealing with emotional connection were indicated as positive factors for high levels of relationship quality which provided useful information for treatment planning and directions for couples therapy.

Cultural differences developed in families of origin, norms of gay people, or racial differences were described as challenges toward better relationship quality. Although no 
qualitative response directly supported the different norms of attachment in the Taiwanese/Chinese or American cultures, participants described significant differences reflected in gender roles, different understanding of social phenomena, values, norms and standards for romantic relationships, and the sense of beauty and body image which may impact quality of romantic relationships. 


\section{CHAPTER FOUR \\ DISCUSSION}

The purpose of this study was to investigate levels of depressive moods and quality of romantic relationships of participants with different attachment styles between Americans and Taiwanese/Chinese international students in the United States. This study also targeted the potentially different experiences in romantic relationships and depressive moods between American participants and participants from the Chinese/Taiwanese culture.

\section{Findings and Interpretation}

Results of this study are partially supportive of the first hypothesis in which American college students reported significant differences in the combined linear dependent variable of the quality of romantic relationships and levels of depressive moods from Taiwanese/Chinese international students. The results of the follow-up ANOVA indicated significantly higher levels of relationship quality of American students than Taiwanese or Chinese international students. When responses from anxiously and avoidantly attached participants were analyzed separately, the results were consistent with the primary findings from the follow-up ANOVA. More specifically, anxiously attached American students reported significantly higher levels of relationship quality than anxiously attached Taiwanese or Chinese international students.

Consistent with those findings, American students with avoidant attachment reported significantly higher levels of relationship quality than Taiwanese or Chinese international students with attachment avoidance. Ethnicity or home country was a significant independent variable for levels of relationship quality. While Yi, Lin, and Kishimoto (2003) found depression to be one of the top three concerns for international students, ethnicity or home country was not found to be a significant independent variable for depressive moods in this 
dissertation research. American students and Taiwanese or Chinese international students reported similar levels of depressive moods regardless of their levels of attachment avoidance or anxiety.

Upon further exploration of the four attachment styles, significant results were determined in relationship quality and depressive moods between Taiwanese or Chinese students and U.S. - born students. Among Taiwanese or Chinese international students, participants with secure attachment indicated significantly higher levels of relationship quality than participants with fearful attachment style. Similarly, participants with a preoccupied attachment style indicated significantly higher levels of relationship quality than participants with a fearful attachment style. Interestingly, Taiwanese or Chinese participants with secure and preoccupied attachment styles indicated similar levels of relationship quality. Consistent with this finding among Taiwanese or Chinese participants, American participants with secure attachment indicated significantly higher levels of relationship quality than participants with fearful attachment. Participants with secure and insecure attachment styles indicated similar levels of relationship quality. Only fearfully attached participants reported significantly lower levels of relationship quality than securely attached participants.

Significant differences were also found in levels of depressive moods between participants with different attachment styles as categorized by the model of four-categorical attachment theory (Bartholomew, 1990; Bartholomew \& Horowitz, 1991). Participants with secure attachment indicated significantly lower levels of depressive moods than participants with fearful attachment. Dismissive individuals (avoidant attachment style according to Ainsworth's research, 1978) indicated significantly higher levels of depressive moods than preoccupied individuals. Fearful American participants also indicated significantly higher 
levels of depressive moods than preoccupied American participants (anxious attachment style in Ainsworth's speculation, 1978). Based on the findings of Muris, Mayer, and Meesters (2000) and Bifulco, Moran, Ball, and Bernazzani (2002), individuals with insecure attachment styles were more likely to exhibit depressive moods. The findings of this study expanded the cultural ramification of attachment theory, in which American students reported significantly higher levels of relationship quality than Taiwanese or Chinese international students with the same level of attachment anxiety. In contrast to the original theory and previous research (e.g., Carnelley, Pietromonaco, \& Jaffe, 1994), depressive moods were not found to be significantly different between participants with different levels of attachment avoidance or attachment anxiety in either the American student group or the Taiwanese or Chinese international student group, despite the findings of Carnelley, Pietromonaco, and Jaffe (1994) who determined that mildly depressive college female students tended to endorse higher levels of fearful or preoccupied attachment.

Furthermore, findings of this study on American college students partially supported Hazan and Shaver's (1987) research which demonstrated that securely attached individuals also endorsed significantly higher levels of relationship quality than insecure attachment styles. More specifically, American participants with secure attachment indicated significantly higher levels of relationship quality than American participants with fearful attachment. However, securely attached and insecurely attached (preoccupied, dismissive, and fearful; Boartholomew \& Horowitz, 1991) American college students indicated similar levels of relationship quality, while only fearfully attached participants reported significantly lower levels of relationship quality than securely attached participants. 
In a similar outcome, Taiwanese or Chinese international students with a secure attachment indicated significantly higher levels of relationship quality than participants with a fearful attachment style. This finding supported Wang and Mallinckrodt's (2006) results, in which male Taiwanese college students endorsed significantly more frequent attachment anxiety in their ideal romantic relationships than their U.S. counterparts. Furthermore, Wang and Mallinckrodt found that female Taiwanese college students endorsed significantly higher levels of attachment avoidance than their U.S. counterparts. In this study, securely attached Taiwanese or Chinese international students who were involved in romantic relationships during the time of the study indicated similar levels of relationship quality as Taiwanese or Chinese international students with preoccupied attachment.

Additionally, Taiwanese or Chinese international students with secure and preoccupied attachment styles indicated significantly higher levels of relationship quality than participants with a fearful attachment style. In findings that were partially consistent with attachment theory and previous research findings (Pistole, 1989; Shemmings 2006), securely attached participants indicated significantly higher levels of relationship quality than fearfully attached participants. Although consistent with Li and Chan's (2012) meta-analysis, participants with a preoccupied attachment style indicated significantly higher levels of relationship quality than participants with a fearful attachment style among Taiwanese or Chinese international students. Findings among Taiwanese international students participating in this study were inconsistent with most previous findings (e.g., Stein, Siefert, Stewart, \& Hilsenroth, 2011) and the theory (Hazan \& Shaver, 1987). Results of this study specifically supported the normalization or neutral effect of attachment anxiety (preoccupied attachment style) and attachment avoidance 
(dismissive attachment style) in the Taiwanese or Chinese culture and the negative impact of fearful attachment.

The non-significant effect of attachment anxiety or avoidance on levels of depressive moods among dating, cohabiting, or married Taiwanese or Chinese international students further extended Wang and Mallinckrodt's (2006) findings, because Taiwanese participants in their study were predominantly college students in Taiwan who were not involved in romantic relationships. Findings for similar levels of relationship quality in Taiwanese or Chinese international students with secure, preoccupied, or dismissive attachment styles also supported the potential impact of cultural differences on relationship quality. This view is further supported in findings regarding American students who do not exhibit a similar phenomenon. It should be specifically noted that Taiwanese or Chinese participants with preoccupied attachment indicated slightly higher levels of relationship quality than securely attached participants. Preoccupied or dismissive attachment styles were hypothesized as insecure attachment styles (Bartholomew \& Horowitz, 1991) which were postulated to be associated with higher levels of depressive moods and lower levels of relationship quality. However, the Chinese cultural norm of frequent assurance or expressive affection-seeking behaviors (behaviors related to anxious attachment; Ainsworth, 1989; Bowlby, 1979, Ho et al., 2010) and withholding of detrimental information from significant others (behavior of attachment avoidance, Ainsworth, 1989; Hazan \& Shaver, 1987) may not be necessarily perceived as negative by individuals or their partners in mainstream Chinese culture. Because anxious and avoidant attachment behaviors are normalized in Chinese culture, such behaviors thus might not influence relationship quality as negatively as the theory or previous research findings would indicate (e.g., Forsythe, Romano, Jensen, \& Thorn, 2012). 
This finding further confirmed the existence of cultural differences in relationship quality based on responses from participants with current involvement in romantic relationships. In terms of the differences related to ethnic or home country, participant responses confirmed significant differences in the combined variable of depressive moods and relationship quality between American and Taiwanese or Chinese international students. Although levels of depressive moods did not differ between Taiwanese or Chinese international students and American students, the significantly lower levels of relationship quality raised the importance of the issues for international students. Furthermore, significantly lower levels of relationship quality of fearfully attached participants than securely attached participants across both cultures supported the importance of attention in treating fearful attachment given that these findings supported the significant difference in levels of relationship quality.

Nonetheless, the measure for relationship quality, Quality of Relationship Inventory (QRI), has not been validated in the Taiwanese and Chinese international student populations to isolate possible different cultural expectations of positive romantic relationships. Hence, the results should be interpreted with caution. Although Taiwanese or Chinese international students reported significantly lower levels of relationship quality than American students, the significant difference might result from different beliefs regarding ideal romantic relationships between these two groups. The lower levels of relationship quality may also be interpreted as an adjustment issue because most Taiwanese or Chinese students have resided in the U.S. for less than ten years, and this was a significantly shorter time span than U.S. students and thus may account for many more adjustment challenges.

After conducting a thematic analysis (Boyatzis, 1998), certain qualitative responses confirmed the quantitative findings of this dissertation research. For example, participants 
identified a negative association between relationship quality and emotional and physical distance, a lack of communication (attachment avoidance), and depressive moods. Attachment anxiety as identified in participant descriptions demonstrated a link between the activation of attachment anxiety and related poor relationship quality.

Extending the findings from the quantitative results and the qualitative responses of participants provided more factors or taboos associated with relationship quality, which may deserve further research effort and provide specific information about cultural differences. Participants expressed factors which significantly influenced their quality of romantic relationships in their experiences which could be categorized into five levels. These were characteristics of partner (characteristic), similarities and compatibility of values (characteristic), gender roles, expressions of affection (relationship), norms of family of origin, expectations within certain populations (the gay population), cultural background (systematic), and employment, health, and housing (environmental).

Although a large number of participants recognized the significance of positive communication in their relationships, a number of communication taboos were also identified, such as previous relationships, betrayals, insulting or degrading language, cursing, divorce, partner's family, deployment in the military, attractiveness to the opposite sex, masturbating, or sex. Considering the significant ethnic difference in relationship quality between Taiwanese or Chinese international students and American students, the cultural differences in the model of positive communication and taboos in these two populations should be carefully explored in counseling or further research.

Furthermore, a number of Caucasian Americans indicated no impact of cultural factors on their relationship quality. Similarly, participants who were involved with a partner of the 
same race, culture, or country of origin did not report any cultural impact. Other participants described the interactions between relationship quality and norms or values from their family of origin, the mainstream American culture, and media that influenced their relationship quality. Specifically, participants reported negative impact of the high divorce rate and religious reservations in the U.S. Furthermore, the myth conveyed in the U.S. media and general culture about the necessity of everlasting passionate love, certain weight, or appearance was identified as having a negative impact on romantic relationships. Treatment attention could focus on communication, development of trust, collaboration in problem-solving, negotiation, and understanding and dealing with the emotional connection based on these qualitative results. Among participants who were engaged in a cross-racial or cross-cultural relationship, genuine curiosity, interest in learning new cultures, understanding, acceptance, and respect for the partner's culture were identified as positive factors for better relationship quality.

\section{Theoretical and Clinical Implications of the Findings}

Attachment focused therapists have proposed methods for providing individual or couples therapy for individuals experiencing attachment distress. Brisch (2011) illustrated the case of a woman suffering from severe depression after her son announced his decision to enter into an early marriage at age 21 . Although her depression appeared to have no cause, Brisch related it to her history of being a parentified first-born adult child whose attachment needs were neglected because she had to take care of younger siblings from early childhood. According to Brisch, acute or severe depression occurred when this client's attachment figure, her adult son, announced his upcoming unavailability resulting from his plan to marry. The positive outcome of treatment was noticeable after the client received inpatient and intense psychotherapy with available attachment figures, such as the unit nurse and the therapist, Brisch. This example 
vividly illustrates the significance and relevance of attachment-focused psychotherapy during the treatment of depression.

Given that international students frequently experience depressive moods while studying in the U.S., the finding of this study further confirmed and highlighted the significant differences between the levels of the linear variable of depressive moods and relationship quality of domestic students and international students from Taiwan or China. According to Brisch, provision of a secure base (Bowlby, 1988), and accessibility of attachment figures reduced levels of depression. Thus clinicians should pay more attention to the development of corrective emotional experiences (Alexander \& French, 1946, p. 294) and secure bases of attachment when treating both American students and Taiwanese and Chinese international students (Wang \& Ratanasiripong, 2010). Integrating attachment and person-centered theories (Bowlby, 1988; Rogers, 1951), effective treatment can encompass provision of congruent and explicit emotional support and validation, consistent and regular appointments, development of availability of additional support between sessions when needed and appropriate, offering information about resources for mental health emergencies, and encouragement of client self-efficacy and motivation in the development of attachment with other individuals in natural support systems, such as colleagues, roommates, and neighbors.

Based on the findings of this study, clinicians should especially facilitate the exploration of meaning, emotions, and behaviors in attachment models of more anxiously attached individuals. In order to ensure multicultural competence in the treatment of international students, therapists should conduct a self-assessment and seek consultation or supervision regarding potential biases against anxious attachment, which was more commonly reported by the international students in this study. I have observed different verbal and nonverbal 
demonstrations of affection between Taiwanese and Chinese international students and domestic students. Generally, verbal assurance of partner positive qualities and abilities among Taiwanese and Chinese international students are not as common as in domestic students and anxious attachment was not determined to be associated significantly with different levels of relationship quality. Because attachment anxiety was found to be associated with lower relationship quality and client self-value has to be postulated to different attachment styles (Bartholomew \& Horowitz, 1991), promoting client self-value and self-esteem can be effective in fostering secure and functional working models of attachment. Applications of established and validated screening measures may enhance treatment effectiveness. After the initial working alliance is established (Bordin, 1979), screening measures could be used to obtain more subjective information about client attachment styles, interpersonal patterns, established schemata, such as Experiences in Close Relationship Scale-Short Form (ECR-S; Wei, Russell, Mallinckrodt, \& Vogel, 2007), The Inventory of Interpersonal Strengths (IIS; Hatcher\& Rogers, 2009), Inventory of Interpersonal Problems (IIPI-64; Alden, Wiggins, \& Pincusm, 1990; Horowitz, Rosenberg, Baer, Ureño, \& Villaseñor, 1988), Young Schema Questionnaire (YSQ, Young \& Brown, 1994), or the Dyadic Adjustment Scale (Spanier, 1976) may provide client cognitive and vocabulary frameworks for individual attachment styles, schemata, and expectations in terms of agreement on values between romantic partners.

Treatment may also focus on prompting clients to identify positive self traits, facilitating client positive self-talk, challenging maladaptive schemata, such as beliefs in self-incompetence in sustaining romantic relationships (dependence/incompetence), inhibition in showing affection to a romantic partner (emotional inhibition), holding very high standards for self-performance in relationships (unrelenting standards/hyper-criticalness), not deserving of love (failure), 
obsessively seeking assurance from other (approval/recognition seeking), and excessively involving emotions with others (enmeshment, cognitive behavioral therapy, CBT; Young, 1988). Furthermore, stability, availability of affection, and desire of others to provide support were also postulated to be distinctive among different attachment styles. Thus, therapists could emphasize challenging maladaptive schemata associated with mis-presumptions of others in the desirability for provision of affection. Certain behaviors resulting from maladaptive schemata can also be discussed, such as the belief in the necessity of harsh punishment when people make mistakes (punitiveness) and presumptions that others will manipulate or abuse (mistrust/abuse).

Similarly, attachment avoidance was determined to contribute a significant amount of variance in depressive moods in this dissertation study. This finding raised the possible issue of treating avoidant attachment as part of treating depression. Considering that individuals with avoidant attachment frequently embrace beliefs about the vulnerability of self, overindependence of self, and unreliability of support from others, maladaptive schemata related to these two misassumptions should be gently challenged in the CBT and attachment informed model of treatment (Bartholomew \& Horowitz, 1991; Young, 1988). While mistrust may emerge from an Asian client toward a Caucasian therapist at this stage, the therapist could also discuss his or her willingness to comprehend and learn the client's cultural, racial, and ethnic background. This can be accomplished through the use of attentive listening and modest inquiries to communicate therapist understanding and empathy in the initial stage of developing a well-connected, understanding, accepting, and collaborative therapeutic relationship. It may be particularly effective in treating clients with insecure attachment in the process of development of mutual comfort in assessing biases by stressing therapist openness in listening to client acknowledgement of potential biases and encouraging the client to verbalize perceived potential 
biases or stereotypes from the therapist. Given that Bowlby (1982) postulated that attachment style can influence interpersonal behaviors, interpersonal patterns of the client and therapist relationship can be assessed and discussed within the framework of a secure and supportive therapeutic relationship.

After the therapist establishes rapport, and engages the client with genuine acceptance, positive regard, and contains client emotional distress, the therapist can then assess and confirm client readiness to explore his or her parental impact on his or her romantic relationships. It is then possible to begin discussing the therapeutic relationship and client presentations and engagement as a parallel of the client's previous relationship with parents and current romantic relationship (person-centered theory; Rogers, 1951; interpersonal therapy; Klerman, Weissman, Rounsaville, \& Chevron, 1984). The client may also gain insight, which is frequently a treatment goal for dynamic oriented therapists (Brody, 1988), in exploring and identifying relationship patterns. If CBT guides treatment, the client may benefit from being challenged in his or her beliefs in the inevitability of self- vulnerability of emotional harm (vulnerability to harm or illness), belief in alienation of self or no possibility of belonging within a group (social isolation/alienation), and doubts about controlling emotions (insufficient self-control/selfdiscipline). Therapist models of support, emotional availability, and transparency for the client can also facilitate client behavioral change along with resulting anxiety or resistance in applying unfamiliar attachment behaviors (social learning theory; Bandura, 1977b). Client resistance can be interpreted as a natural defense in preserving and protecting the self (Gestalt, Engle \& Holiman, 2002). Thus, open discussions and information about this theoretical hypothesis can be provided to facilitate client understanding of the merit of resistance instead of feeling frustrated about failure in collaboration with the therapist. Further assessment of client reactive feelings 
and potential modification of the time frame or strategy adjustment in embracing new thoughts or behaviors in the process of change may also prove beneficial.

Clinicians may utilize explanation, immediacy, and information giving more frequently with Asian clients, as Sue and Sue $(2003,2007)$ stressed the treatment preference of Asians in more active-directive forms of therapy. Using the Gestalt technique (Burley, 2012), which is similar to the immediacy technique and guiding questions, may improve client self-awareness in emotional presentations in facial expressions and body language as well as in verbal and nonverbal behaviors. These techniques may also prove useful in therapy with anxiously attached clients who excessively seek validation, verbalize self-doubt, or maintain emotional distance or indifference when dealing with significant relationship ruptures. Information given can be applied to help the client understand different attachment styles and assist the client to objectively assess attachment patterns, techniques in adaptive changes, such as using the selfmanagement technique to reduce frequency of approval seeking or tracking frequency of applications of self-soothing techniques (CBT, Cormier, Nurius, \& Osborn, 2003, 2009).

Given that the general Asian culture in which Taiwanese and Chinese international students are rooted is more collectivistic than American culture (Sue \& Sue, 2003, 2007), the social context in most cases may be more significant for Taiwanese or Chinese international students. Thus, clients may benefit from more therapist facilitation in discussions about norms or values from their family of origin, and mainstream American culture, as well as myths of everlasting passion or body image in romantic relationships as conveyed by the media. Particularly among participants who were engaged in cross-racial or cross-cultural relationships, assisting the partner to facilitate or develop genuine curiosity, interest in learning new cultures, 
understanding, acceptance, and respect for the partner's culture were identified as positive factors that may enhance relationship quality.

Because a number of participants identified communication as a salient factor for relationship quality in their qualitative responses, emphasis on perspectives and skills in promoting compatible and effective expression of affection may promote more effective communications within couples and further improve their relationship quality. For example, The Five Love Languages: The Love That Lasts of Chapman (1992, 1995, 2004, \& 2010) may be used as an additional reference for couples reporting difficulty in identifying or verbalizing differences in expectations and effective communication of love. Chapman defined the five primary love languages to be words of affirmation, acts of service, quality time, receiving gifts, and physical touch. The brief exploratory test of individual primary means of perceiving affection from a partner can be completed by clients and the categories used as an introduction to further discussion and negotiation of individual differences. This test may also lead to the development of a behavioral structure for improvement of effective communication and reception of affection within couples.

Client motivation and self-confidence may be buffered when clients actively engage in perceiving and assessing their own progress. Integrating the motivational interview and introducing the natural fluctuation of progress between the stages of change (Prochaska \& DiClemente, 1983) can protect the client from frustration or the inclination to give up when positive changes in fostering secure attachment experience are made, but then pre-existing attachment patterns may be reverted to. Attachment focused role-play may also be facilitated to assist individuals in practicing new verbal or behavioral presentations while therapists provide validation, process comments, and encourage discussions intended to explore the here-and-now 
feelings, fear, and anxiety in developing new ways of relating and attachment experience.

Clients can also be motivated through different levels of therapist facial expressions, tone of voice, volume, and gesture according to client verbalized preference or identified common ways of client perception of encouragement. Clients may need more animated therapist encouragement during a period of client age regression. However, an emotionally reserved client or one who struggles with perceived feelings of maturity may receive therapist validation through only a slight change of tone, gesture, or facial expression. Taiwanese and Chinese clients may not expect as many verbal validations as American clients (Wang, 1990), but rely more on nonverbal validation, such as therapist open-arm gestures, good eye contact, smiles, or leaning of the upper body toward the client.

Client flexibility and understanding of the development of his or her attachment style may be nurtured by introducing a new attachment figure or evolutionary values of different attachment styles and then assessing the pros and cons of presenting with a different attachment style in different circumstances (Fraley \& Brumbaugh, 2004). For instance, an anxiously attached wife may benefit from self-emotional assurance after she confirms the availability and proximity of her husband during a war. She may also extend her survival and reproductive success by gaining proximity, protection, and physical intimacy because of the demonstration and effect of attachment anxiety. However, the same presentation may impede self-growth or emotional stability during a non-war era. Consistently, individuals may attempt attachment avoidant reactions immediately after experiencing traumatic incidents to prevent elevated or potential disabling emotional or behavioral distress of significant others. The reluctance in disclosing traumatic experiences may be functional when the partner is engaging in important tasks for the survival or reproductive needs of the couple, such as collecting food during a 
natural disaster. The preservation of disclosure may also result from cultural norms, such as withholding the most distressing news from close parents/family members in the Taiwanese culture. Therapist elaboration of research findings, verbalizing the limitations of the therapist due to different backgrounds, and inquiring about client beliefs and values in such practices may further facilitate the therapeutic relationship and therapist multicultural competence.

Based on the self-psychology conceptualization, clinicians may direct attention and effort toward being a stable object by playing a parent-like role and providing consistent emotional support while maintaining appropriate therapeutic boundaries (Kohut, 1971). Depending on the length of the romantic relationship and the stage of the couple's interdependence and self-couple identity, clinicians may facilitate couples in describing individual experiences in different stages of the romantic relationships in couple's therapy. Discussions about individual experiences can assist clients in identifying the couple's interactions and causal or linear process of individual presumption (individual attachment style) of certain partner behavior using individual perception based on preexistent attachment models. Assessing the couple's interactions can also improve individual awareness in emotional and behavioral reactions which elicit partner reactions, and may maintain or discontinue pre-established individual working models (Ainsworth \& Bowlby, 1969). For example, dyads with anxiety-avoidance attachment styles may improve the couple's awareness in the seeking and hiding cycles, then negotiate and adjust individual behaviors according to the attachment styles of both partners, and thus further assist each other in developing a secure attachment in the relationship.

The interactions between intra-psychic and relational components are also worthy of clinical attention. The exploration in mirror and idealizing transferences may be crucial for couples to understand and evolve in the potential adjustment of individual expectations (Kohut, 
1971). According to Kohut, mirror transference postulates that the individual self-construct is sustained by continuous high levels of admiration from others; idealizing transference is described as a reflective self-image maintained by constant total attentive behaviors of others. Because a husband tends to hold mirror transference while a wife inclines to embrace idealizing transference, therapist reflection on the presentations of intrapsychic expectations while fostering individual awareness in discussing the underlying desire may further deepen therapeutic relationships (Feldman, 1982). As mentioned in the literature review, object relations theory highlights client developmental needs in developing a sense of self, separating self from the primary attachment figure, and recognizing the gray area between extremely positive and negative self-evaluations (Novaco, 1978). If sensitive parental mirroring and parent-child boundaries for client needs in the development of positive and adaptive self-image are missing, clinicians may facilitate therapeutic cohesiveness and healing by mirroring the emergence of client self-construct and encouraging clients in developing self-encouragement and self-efficacy in relating to their partners.

\section{Strengths and Limitations}

Strengths of this study include the broad geographical area, current involvement of participants in romantic relationships, participation of both U.S. and international students, recruitment of participants at different stages of romantic relationships (dating, committed, cohabiting, and married), inclusion of participants from different regions of the United States, and the mixed methods design. Findings of this study can be generalized to more regions in the U.S. than the broad geographical areas in which participants were residing with few concerns about regional differences. The participation of international students who were studying in the 
U.S. may potentially provide findings that can be generalized and applied to fit the unique needs of international students from Taiwan or China in the U.S.

Additionally, most previous research included only participants who were not involved in romantic relationships (e.g., Wang \& Mallinckrodt, 2006) or at only one stage (e.g., marriage; Fagan, 2009) and thus the generalizability of the findings may be limited to non-dating or married participants. The mixed method research design provided statistically significant and generalizable findings, while the qualitative results provided additional confirmation for the quantitative findings, significant topics in treating couples or individuals with relationship issues, and contextual impact manifested in their romantic relationships (e.g., the impact of opinions or involvement of family of origin, mainstream American culture, media influence, or cultural factors unique to international or gay populations).

Exclusive online data collection, moderate sample size, and the use of English surveys are the main limitations of this research. Because the only methods in completion of the designation surveys were online, students who were not computer literate or had access to a computer with Internet connections were less likely to participate in this research. Due to the limitation of research funding, data were collected online via student online groups on Facebook, Google Groups, Yahoo Groups, and discussion boards sponsored by student associations, staff at offices of international students, student activities, student affairs, or academic departments. Numbers of participants from each educational institution were different due to staff and student willingness, and thus, most participants resided in the Midwest area of the United States. Furthermore, although a larger number of international student participants was desirable, U.S. participants outnumbered international student participants due to the small size of the Taiwanese/Chinese student populations in these universities. 
Ethnic differences in both of the international student and the American groups might be omitted by including both Taiwanese and Chinese international students in one group and American students in one group. Due to the consideration of potential numbers of participants, this dissertation author had to use a broader category. Additionally, most the U.S. students were undergraduate students, and most of the international students were graduate students. The different academic levels of the two groups might confound the findings.

Furthermore, because the international students were the target participants, using Mandarin instruments validated with Taiwanese or Chinese samples may have increased the likelihood of international student participation or reduced the numbers of excluded data due to conflicting responses. Due to a lack of literature on the validation of the Quality of Relationships Inventory (QRI), the reliability of QRI may be hindered in assessment of relationships of the Taiwanese or Chinese international student populations. Researchers may improve the reliability of the QRI by conducting a study to validate the reliability and validity in application to the Taiwanese or Chinese international student populations. Additionally, the six open-ended questions were presented to participants before the quantitative measures (QRI, CES-D, or ECR-S). The sequence might elicit characteristics of participant responses when participants looked at the open-ended questions before filling out the quantitative measures.

\section{Suggestions for Future Study}

Future research can be developed to extend the number of student participants and include paper-and-pencil data collection if resources permit. Extending the research invitation to in-person communication interfaces and cyber boards accessible to the entire student populations of the randomly selected universities may further strengthen the representativeness of these research findings. Given that cultural differences have been well documented regarding 
romantic relationships, future qualitative research can be conducted to explore the different components of preferred romantic relationships between American students and Taiwanese or Chinese college students. Then a more culturally sensitive measure or supplementing self-report measures by collecting trained observer ratings, conducting interviews, or focus groups can be developed based on the findings of the qualitative investigation.

To concisely understand the difference between the two groups (Taiwanese/Chinese international students and the U.S. students), recruiting enough participants for each category (e.g. Taiwanese international students or African American students) may reduce the potentially variance within the current groups. Additionally, balancing the numbers of students in each academic level (undergraduate and graduate) may also reduce the potential confounding variance in age and romantic experiences.

Longitudinal or pre-test posttest research can be applied to assess baselines of student depressive moods. More potential intervening variables can be collected and the variability of student moods can be more controlled, such as time frame in a semester (e.g., finals week vs. first week of classes). Research can be extended by further interviews with students who report significantly high, low, and average levels of depressive moods or quality of romantic relationships. This further research may extend our understanding and lead to more effective treatment approaches for students who experience depressive moods, attachment distress, or romantic issues during their university careers. Additionally, the six open-ended questions can be presented to participants after the quantitative measures (QRI, CES-D, or ECR-S) to avoid eliciting characteristics of participant responses when participants looked at the open-ended questions before filling out the quantitative measures. 


\section{Conclusion}

The findings of this dissertation have extended the understanding of both similar and different associations between depressive moods, relationship quality, and avoidant or anxious attachment styles between Taiwanese or Chinese international students and American students. For example, both Taiwanese/Chinese and American participants with secure attachment indicated significantly lower levels of depressive moods than participants with fearful attachment. Meanwhile, cultural differences were detected between the two populations because only Taiwanese or Chinese international students with secure and preoccupied attachment styles indicated significantly higher levels of relationship quality than participants with fearful attachment style. Only dismissive American participants (avoidant attachment style in Ainsworth's speculation, 1978) indicated significantly higher levels of depressive moods than preoccupied individuals.

Findings for similar levels of relationship quality of Taiwanese or Chinese international students with secure, preoccupied, or dismissive attachment styles also supported the potential impact of cultural differences on relationship quality given that a similar phenomenon was not detected among American students. The Chinese cultural norms of frequent assurance or expressive affection seeking behaviors or avoidant behaviors (withholding extremely distressing experiences from significant others) may contribute to the different findings among these populations. Thus, the findings further raised the need for future research focusing on the relationships between depressive moods, relationship quality, and contextual factors (e.g., interactions, impact of family of origin, cultural adjustment, American culture, or norms and expectations within more specific populations within the Taiwanese/Chinese or American populations). 
In conclusion, the present study provided partial support for the theoretical base of attachment theory for therapeutic intervention in relationship issues and depressive moods for both American college students and Taiwanese/Chinese international students. The findings also demonstrate the need for further research into contextual factors for Taiwanese/Chinese international populations and the clinical needs of American students with avoidant attachment. Hence, this study has extended the foundation for current treatment and demonstrated the need for further research to promote multicultural competence for these two target populations. 


\section{REFERENCES}

Abakoumkin, G., Stroebe, W., \& Stroebe, M. (2010). Does relationship quality moderate the impact of martial bereavement on depressive symptoms? Journal of Social and Clinical Psychology, 29(5), 510-526.

Abouguendia, M., \& Noels, K. A. (2001). General and acculturation-related daily hassles and psychological adjustment in first- and second-generation South Asian immigrants to Canada. International Journal of Psychology, 36(3), 163-173.

Abu-Bader, S. H. (2010). Advanced \& Multivariate Statististical Methods for Social Science Research: With A Complete SPSS Guide. Chicago, Illinois: Lyceum Books.

Adler, P. (1975). The transitional experience: An alternative view of culture shock. Journal of Humanistic Psychology, 15, 13-23.

Ainsworth, M. D. S. (1989). Attachment beyond infancy. American Psychologist, 44, 709-716.

Ainsworth, M. S., \& Wittig, B. (1969). Attachment and exploratory behavior of one-year-olds in a strange situation. In B. Foss (Ed.), Determinants of infant behaviour (Vol.4, pp. 111136). London: Methuen.

Allen, J. P., \& Miga, E. M. (2010). Attachment in adolescence: A move to the level of emotion regulation. Journal of Social and Personal Relationships, 27, 181-190.

Anisworth, M. S. (1989). Attachments beyond infancy. American Psychologist, 44(3), 709-716. Ainsworth, M. S., \& Bowlby, J. (1991). An ethological approach to personality development. American Psychologist, 46(4), 333-341.

American Psychiatric Association. (2000). Diagnostic and Statistical Manual of Mental Disorders, 4th Edition, Text Revision (DSM-IV-TR, $4^{\text {th }}$ ed.) Washington, D.C.: Author. 
American Psychological Association. (2002). Guidelines on Multicultural Education, Training, Research, Practice, and Organizational Change for Psychologists. American Psychological Association. Retrieved from http://www.apa.org/pi/oema/resources/policy/multicultural-guidelines.aspx\#

Anand, R. P. (2010). The formation of international organizations and India: A historical study. Leiden Journal of International Law, 23(1), 5-21.

Bäckström, M., \& Holmes, B. M. (2007). Measuring attachment security directly: A suggested extension to the two-factor adult attachment construct. Individual Differences Research, 5(2), 124-149.

Banai, E., Mikulincer, M., \& Shaver, P. R. (2005). "Selfobject" needs in Kohut's self psychology: Links with attachment, self-cohesion, affect regulation, and adjustment. Psychoanalytic Psychology, 22(2), 224-260.

Barlett, H. P., Simonite, V., Westcott, E., \& Taylor, H. (2000). A comparison of the nursing competence of graduates and diplomates from UK nursing programmes. Journal of Clinical Nursing, 9, 369-381.

Barnes, D. L. (2006). Postpartum depression: Its impact on couples and marital satisfaction. Journal of Systemic Therapies, 25(3), 25-42.

Barresi, J. (1998). On becoming a person. Retrieved from http://jbarresi.psychology.dal.ca/Papers/beperson.html

Barth, J., Volz, A., Schmid, J., Kohls, S., von Kănel, R., Znoj, H., \& Saner, H. (2009). Gender differences in cardiac rehabilitation outcomes: Do women benefit equally in psychological health? Journal of Women's Health, 18(12), 2033-2039. 
Bartholomew, K. (1990). Avoidance of intimacy: An attachment perspective. Journal of Social and Personal Relationships, 7, 147-178.

Bartholomew, K., \& Horowitz, L. (1991). Attachment styles among young adults: A test of the four category model. Journal of Personality and Social Psychology, 61, 226-244.

Bartholomew, K. S., \& Shaver, P. (1998). Methods of assessing adult attachment: Do they converge? In J. A. Simpson \& W. S. Rholes (Eds.), Attachment Theory and Close Relationships (pp. 25-45). New York: Guilford Press.

Beach, S. R. H., Dreifuss, J. A., Franklin, K. J., Kamen, C., \& Gabriel, B. (2008). Couple therapy and the treatment of depression. In A. S. Gurman (Ed.). Clinical handbook of couple therapy (4th ed., pp. 545-566). New York: Guilford Press.

Beck, A. T., \& Steer, R. A. (1993). Manual for the Beck Depression Inventory. New York: Psychological Corporation.

Beck, A. T., Steer, R. A., \& Brown, G. K. (1987). Manual of the Revised Beck Depression Inventory manual ( $2^{\text {nd }}$ ed.). San Antonio: Psychological Corporation.

Beck, A. T., Steer, R. A., \& Brown, G. K. (1996). Beck Depression Inventory manual (2 ${ }^{\text {nd }}$ ed.). San Antonio: Psychological Corporation.

Bifulco, A., Moran, P. M., Ball, C., \& Bernazzani, O. (2002). Adult attachment style. I: Its relationship to clinical depression. Social Psychiatry and Psychiatric Epidemiology, $37(2), 50-59$.

Bohart, A., \& Tallman, K. (1999). How clients make therapy work: The process of active selfhealing. In A. S. Gurman \& S. B. Messer (Eds.), Essential Psychotherapies: Theory and Practice (2nd ed., pp. 107-144). New York: The Guilford Press.

Bowlby, J. (1969). Attachment and loss: Vol. 1. Attachment. New York: Basic Books. 
Bowlby, J. (1973). Attachment and loss: Vol. 2. Separation: Anxiety and anger. New York: Basic Books.

Bowlby, J. (1977). The making and breaking of affectional bonds: I. Aetiology and psychopathology in the light of attachment theory. British Journal of Psychiatry, 130, 201-210.

Bowlby, J. (1979). The making and breaking of affectional bonds. London: Tavistock.

Branje, S. J. T., Hale III, W. W., Frijns, T., \& Meeus, W. H. J. (2010). Longitudinal associations between perceived parent-child relationship quality and depressive symptoms in adolescence. Journal of Abnormal Child Psychology, 38(6), 751-763.

Braun, V., \& Clarke, V. (2006). Using thematic analysis in psychology. Qualitative Research in Psychology, 3, 77-101.

Brennan, K. A., Clark, C. L., \& Shaver, P. R. (1998). Self-report measurement of adult attachment: An integrative overview. In J. A. Simpson \& W. S. Rholes (Eds.), Attachment Theory and Close Relationships (pp. 46-76). New York: Guilford Press.

Brennan, K. A., Clark, C. L., \& Shaver, P. R. (1998). Attachment styles and personality disorders: Their connections to each other and to parental divorce, parental death, and perceptions of parental caregiving. Journal of Personality, 66(5), 835-878.

Brown, S. L. (2000). The effect of union type on physiological well-being: Depression among cohabiters versus marrieds. Journal of Health \& Social Behavior, 41(3), 241-255.

Carnegie Foundation of Excellence Teaching. (2010). Carnegie Classification of Institutions of Higher Education. Retrieved from http://classifications.carnegiefoundation.org/descriptions/basic.php 
Carnelley, K. B., Pietromonaco, P. R., \&Jaffe, K. (1994). Depression, working models of others, and relationship functioning. Journal of Personality and Social Psychology, 66(1), 127140.

Cassidy, J. (2008). The nature of the child's ties. In J. Cassidy \& P. R. Shaver (Eds.), Handbook of Attachment: Theory, Research, and Clinical Applications (pp. 3-22). New York \& London: The Guilford Press.

Chapman, G. D. (1992, 1995, 2004, \& 2010). The Five Love Languages. The 5 Love Languages: The Secret to Love That Lasts. Chicago: Northfield Publishing.

Chataway, C. J., \& Berry, J. W. (1989). Acculturation experiences, appraisal, coping, and adaptation: A comparison of Hong Kong Chinese, French, and English students in Canada. Canadian Journal of Behavioural Science, 21(3), 295-309.

Cheng, L. L. (1999). Sociocultural adjustment of Chinese-American students. In C. C. Park \& M. M. Chi (Eds.), Asian-American education: Prospects and challenges (pp. 1-17). Westport, CT: Bergin \& Garvey.

Chentsova-Dutton, Y. E., Tsai, J. L., \& Gotlib, I. H. (2010). Further evidence for the cultural norm hypothesis: Positive emotion in depressed and control European American and Asian American women. Cultural Diversity and Ethnic Minority Psychology, 16(2), 284295.

Cheung, R. Y. M., \& Park, I. J. K. (2010). Anger suppression, interdependent self-construal, and depression among Asian American and European American college students. Cultural Diversity and Ethnic Minority Psychology, 16(4), 517-525.

Chi, I., \& Boey, K. W. (1993). Hong Kong validation of measuring instruments of mental health status of the Chinese elderly in Hong Kong. Clinical Gerontolody, 13, 35-51. 
Chi, I. \& Chou, K. (2001). Social support and depression among elderly Chinese people in Hong Kong. International. Journal of Aging and Human Development, 52(3) 231-252.

Choi, H., \& Marks, N. F. (2008). Marital conflict, depressive symptoms, and functional impairment. Journal of Marriage \& Family, 70(2), 377-390. DOI: 10.1111/j.17413737.2008.00488.x.

Choi, T. (2006). Asian international students' academic adjustment in a U.S. graduate school and Stanton-Salazar's framework. Pacific Asia Education, 18, 51-68.

Chronicle of Higher Education. (2009, November 16). Number of foreign students in U.S. hit a new high last year. Chronicles of Higher Education. Retrieved from http://chronicle.com/article/Number-of-Foreign-Students-in/49142/

Chu, B. C. (2007). Considering culture one client at a time: Maximizing the cultural exchange. Pragmatic Case Studies in Psychotherapy, 3(3), 34-43.

Cohen, J. (1988). Statistical power analysis for the behavioral sciences $\left(2^{\text {nd }}\right.$ ed.). Hillsdale, NJ: Erlbaum.

Conradi, H. J., Gerlsma, C., van Duijn, M., \& de Jonge, P. (2006). Internal and external validity of the Experiences in Close Relationships Questionnaire in an American and two Dutch samples. The European Journal of Psychiatry, 20(4), 258-269.

Conradi, H. J., \& de Jonge, P. (2009). Recurrent depression and the role of adult attachment: A prospective and a retrospective study. Journal of Affective Disorders, 116(1-2), 93-99.

Constantine, M. G., Kindaichi, M., Okazaki, S., Gainor, K. A., \& Baden, A. L. (2005). A qualitative investigation of the cultural adjustment experiences of Asian international college women. Cultural Diversity and Ethnic Minority Psychology, 11, 162-175. 
Cooper, J. B., Meyer, D. D., \& Paul, R. H. (2008). Marriage and early life stressors as correlates for depression and anxiety: Implications for counselors. In G. R. Walz, J. C. Bleuer, \& R. K. Yep (Eds.).Compelling Counseling Interventions: Celebrating VISTAS' fifth anniversary (pp. 49-58). Alexandria: American Counseling Association.

Cramer, D. (2004). Satisfaction with a romantic relationship, depression, support and conflict. Psychology and Psychotherapy: Theory, Research and Practice, 77(4), 449-461.

Cramer, D. (2004). Emotional support, conflict, depression, and relationship satisfaction in a romantic partner. Journal of Psychology, 138(6), 532-542.

Curran, M., Hazen, N., Jacobvitz, D., \& Sasaki, T. (2007). How representations of the parental marriage predict marital emotional attunement during the transition to parenthood. Journal of Family Psychology, 20(3), Special issue: Relational disorders and relational processes in mental health, 477-484.

Davila, J. (2008). Depressive symptoms and adolescent romance: Theory, research, and implications. Child Development Perspectives, 2(1), 26-31.

Defoe, D. (2009, January). International students still flock to U.S. University Business, 12(1), 11.

De Jong Gierveld, J., \& Kamphuis, F. H. (1985). The development of a Rasch-type loneliness scale. Applied Psychological Measure, 9, 289-299.

Dennett, D. C. (1978). Brainstorms. In J. Barresi (1998), on becoming a person. Retrieved from http://jbarresi.psychology.dal.ca/Papers/beperson.html

Derogatis, L. R. (2000). Brief Symptom Inventory (BSI) 18 administration, scoring, and procedures manual. Minneapolis, MN: NCS Pearson. 
Derogatis, L. R. (1992). The Symptom Checklist-90-revised. Minneapolis, MN: NCS Assessments.

Diamond, L. M., \& Fagundes, C. P. (2010). Psychobiological research on attachment. Journal of Social and Personal Relationships, 27(2), 218-225.

Dinero, R. E., Conger, R. D., Shaver, P. R., Widaman, K. F., \& Larsen-Rife, D. (2008). Influence of family of origin and adult romantic partners on romantic attachment security. Journal of Family Psychology, 22(4), Special issue: Public health perspectives on family interventions, 622-632.

D'Rozario, D., \& Choudhury, P. K. (2000). Effect of assimilation on consumer susceptibility to interpersonal influence. Journal of Consumer Marketing, 17(4), 290-307.

Eng, S., Kanitkar, K., Cleveland, H. H., Herbert, R., Fischer, J., \& Wiersma, J. D. (2008). Educational Psychology, 28(5), 535-550.

Fagan, J. (2009). Relationship quality and changes in depressive symptoms among urban, married African Americans, Hispanics, and Whites. Family Relations, 58(3), 259-274.

Fischer, C. T. (2009). Bracketing in qualitative research: Conceptual and practical matters. Psychotherapy Research, 19 (4-5), 583-590.

Fletcher, G. J. O., Simpson, J. A., \& Thomas, G. (2001). The measurement $\mathrm{f}$ perceived relationship quality components: A confirmatory factor-analytic approach. Personality and Social Psychology Bulletin, 16, 340-354.

Forsythe, L. P., Romano, J. M., Jensen, M. P., \& Thorn, B. E. (2012). Attachment style is associated with perceived spouse responses and pain-related outcomes. Rehabilitation Psychology, 57(4), 290-300. 
Fraley, R. C., Waller, N. G., \& Brennan, K. A. (2000). An item-response theory analysis of selfreport measures of adult attachment. Journal of Personality and Social Psychology, 78, $350-365$.

Furukawa, T. (1997). Depressive symptoms among international exchange students, and their predictors. Acta Psychiatrica Scandinavica, 96, 242-246.

Griffin, D., \& Bartholomew, K. (1994a). Metaphysics of measurement: The case of adult attachment. In K. Bartholomew \& D. Perlman (Eds.), Advances in personal relationships, Vol. 5: Attachment processes in adulthood (pp. 17-52). London, England: Jessica Kingsley.

Hallfors, D. D., Waller, M. W., Ford, C. A., Halpern, C. T., Brodish, P., \& Iritani, B. (2004). Adolescent depression and suicide risk: Association with sex and drug behavior. American Journal of Preventive Medicine, 27(3), 224-231. DOI:

10.1016/j.amepre.2004.06.001

Hallfors, D. D., Waller, M. W., Bauer, D., Ford, C. A., \& Halpern, C. T. (2005). Which comes first in adolescence-sex and drugs or depression? American Journal of Preventive Medicine, 29(3)163-170. DOI: 10.1016/j.amepre.2005.06.002

Harwood, E. A. (2008). Attachment, personality, and conflict behaviors in romantic couples: Examining vulnerability to depression. (Doctoral dissertation). Retrieved from Ebscohost database. (2009-99060-046)

Hazan, C., \& Shaver, P. R. (1994). Attachment as an organizational framework for research on close relationships. Psychological Inquiry, 5(1), 1-22.

Hazan, C., \& Shaver, P. R. (1987). Romantic love conceptualized as an attachment process. Journal of Personality and Social Psychology, 52, 511-524. 
He, Y., Zhang, Y., \& Yang, H. (2010). Relationship between adult attachment, self-esteem and social support of college students. Chinese Journal of Clinical Psychology, 18(2), 247249.

Hendershot, C. S., Dillworth, T. M., Neighbors, C., \& George, W. H. (2008). Differential effects of acculturation on drinking behavior in Chinese- and Korean-American college students. Journal of Studies on Alcohol and Drugs, 69(1), 121-128.

Herman, S., Archambeau, O. G., Deliramich, A. N., Kim, B. S. K., Chiu, P. H., \& Frueh, B. C. (2011). Depressive symptoms and mental health treatment in an ethnoracially diverse college student sample. Journal of American College Health, 59(8), 715-720. DOI: $10.1080 / 07448481.2010 .529625$

Herr, N. R., Hammen, C., \& Brennan, P. A. (2007). Current and past depression as predictors of family functioning: A comparison of men and women in a community sample. Journal of Family Psychology, 21(4), 694-702.

Ho, M. Y., Zhang, H., Lin, D., Lu, A., Bond, M. H., Chan, C., \& Friedman, M. (2010). Saving graces: Impact of partner support and maternal attachment on partner attachments in an individualistic and a collectivist context. Asian Journal of Social Psychology, 13(1), 1929.

Holahan, C. J., Moerkbak, M. L., Moos, R. H., Cronkite, R. C., Holahan, C. K., \& Kenney, B. A. (2007). Spousal similarity in coping and depressive symptoms over 10 years. Journal of Family Psychology, 21(4), 551-559.

Horowitz, L. M. Rosenberg, S. E., Baer, B. A., Ureño, G., \& Villaseñor, V. S. (1988). Inventory of interpersonal problems: Psychometric properties and clinical applications. Journal of Consulting and Clinical Psychology, 56(6), 885-892. 
Huang, C. (2009). Stressors, social support, depressive symptoms and general health status of Taiwanese caregivers of persons with stroke or Alzheimer's disease. Journal of Clinical Nursing, 18 (4), 502-511.

Huntington, S. (1996). The Clash of Civilizations and the Remaking of World Order. New York: Simon \& Schuster.

Joiner, T. E., \& Schmidt, N. B. (1998). Excessive reassurance-seeking predicts depressive but not anxious reactions to acute stress. Journal of Abnormal Psychology, 107, 533-537.

Jordan, J. V. (2001). A relational-cultural model: Healing through mutual empathy. Bulletin the Menninger Clinic, 65, 92-103.

Joyner, K., \& Udry, J. R. (2000). You don't bring me anything but down: Adolescent romance and depression. Journal of Health and Social Behavior, 41(4), 369-391.

Kessler, R. C., Andrews, G., Mroczek, D., Ustun, T. B., \& Wittchen, H. U. (1998). The World Health Organization Composite International Diagnostic Interview Short-Form (CIDISF). International Journal of Methods in Psychiatric Research, 7, 171-185.

Kim, B. C. (1996). Korean families. In M. McGoldrick, J. Giordano, \& J. K. Pearce (Eds.), Ethnicity and family therapy (2nd ed., pp. 281-294). New York: Guilford Press.

Kim, B. S. K., Atkinson, D. R., \& Yang, P. H. (1999). The Asian Values Scale: Development, factor analysis, validation, and reliability. Journal of Counseling Psychology, 46, 342352.

Kim, B. S. K., Yang, P., Atkinson, D. R., Wolfe, M. M., \& Hong, S. (2001). Cultural value similarities and differences among Asian American ethnic groups. Cultural Diversity and Ethnic Minority Psychology, 7(4), 343-361. 
Kim, G., DeCoster, J., Huang, C., \& Chiriboga, D. A. (2011). Cultural Diversity and Ethnic Minority Psychology, 17(4), 381-396.

Kim, H. E., Edwards, A. B., Sweeney, K. A., \& Wetchler, J. L. (2012). The effects of differentiation and attachment on satisfaction and acculturation in Asian-White American international couple relationships: Assessment with Chinese, South Korean, and Japanese partners in relationships with white American partners in the United States. American Journal of Family Therapy, 40(4), 320-335.

Kim, K. C., Hurh, W. M., \& Kim, S. (1993). Generation differences in Korean immigrants' life conditions in the United States. Sociological Perspectives, 36(3), 257-270.

Kim, S. Y. (2010). Do Asian values exist? Empirical tests of the four dimensions of Asian values. Journal of East Asian Studies, 10(2), 315-344.

Kitayama, S., Mesquita, B., \& Karasawa, M. (2006). Cultural affordances and emotional experience: Socially engaging and disengaging emotions in Japan and the United States. Journal of Personality and Social Psychology, 91(5), 890-903.

Kline, S. L., Horton, B., \& Zhang, S. (2008). Communicating love: Comparisons between American and East Asian university students. International Journal of Intercultural Relations, 32(3), 200-214.

Knobloch, L. K., \& Solomon, D. H. (1999). Measuring the sources and content of relational uncertainty. Communication Studies, 50, 261-278.

Knobloch, L. K., \& Solomon, D. H. (2002a). Information seeking beyond initial interaction:

Negotiating relational uncertainty within close relationships. Human Communication Research, $28,243-257$.

Knobloch, L. K. (2007a). The dark side of relational uncertainty: Obstacle or opportunity? 
In B. H. Spitzberg \& W. R. Cupach (Eds.), The dark side of interpersonal communication (2nd ed., pp. 31-59). Mahwah, NJ: Lawrence Erlbaum.

Knobloch, L. K., \& Knobloch-Fedders, L. M. (2010). The role of relational uncertainty in depressive symptoms and relationship quality: An actor-partner interdependence model. Journal of Social and Personal Relationships, 27(1), 137-159.

Kochanska, G. (2001). Emotional development in children with different attachment histories: The first three years. Child Development, 72(2), 474-490.

Kohut, H. (1966). Forms and transformations of narcissism. Journal of the American Psychoanalytic Association, 14(2), 243-272.

Kohut, H. (1971). The analysis of the self. New York: International Universities Press.

Kouros, C. D., Merrilees, C. E., \& Cummings, E. M. (2008). Marital conflict and children's emotional security: in the context of parental depression. Journal of Marriage and Family, 70(3), 684-697.

Krohne, H. W., Schmukle, S. C., Spaderna, H., \& Spielberger, C. D. (2002). The State-Trait Depression Scales: An international comparison. Anxiety, Stress \& Coping: An International Journal, 15(2), 105-122.

Kuo, B. C. H. (2004). Interdependent and relational tendencies among Asian clients: Infusing collectivistic strategies into counseling. Guidance \& Counseling, 19(3), 158-162.

Law, R. W., \& Sbarra, D. A. (2009). The effects of church Attendance and marital status on the longitudinal trajectories of depressed mood among older adults. Journal of Aging and Health, 21(6), 803-823. 
Lee, A., \& Hankin, B. L. (2009). Insecure attachment, dysfunctional attitudes, and low selfesteem predicting prospective symptoms of depression and anxiety during adolescence. Journal of Clinical Child \& Adolescent Psychology, 38(2), 219-231.

Leese, M. (2010). Bridging the gap: Supporting student transitions into higher education. Journal of Further and Higher Education, 34(2), 239-251.

Lemmens, G. M. D., Buysse, A., Heene, E., Eisler, I., \& Demyttenaere, K. (2007). Marital satisfaction, conflict communication, attachment style and psychological distress in couples with a hospitalized depressed patient. Acta Neuropsychiatrica, 19(2), 109-117.

Leong, F. L., \& Chou, E. L. (1994). The role of ethnic identity and acculturation in the vocational behavior of Asian Americans: An integrative review. Journal of Vocational Behavior, 44, 155-172.

Leong, E. L., \& Gim-Chung, R. H. (1995). Career assessment and intervention with Asian American. In F. T. L. Leong (Ed.), Career development and vocational behavior of racial and ethnic minorities (pp. 193-226). New Jersey: Lawrence Erlbaum Associates.

Leong, F. L., \& Sedlacek, W. E. (1986). A comparison of international and U.S. students' preferences for help sources. Journal of College Student Personnel, 27, 426-430.

Li, T., \& Chan, D. K. (2012). How anxious and avoidant attachment affect romantic relationship quality differently: A meta analytic review. European Journal of Social Psychology, 42(4), 406-419.

Li, L. W. \& Liang, J. (2007). Social exchanges and subjective well-being among older Chinese: Does age make a difference? Psychology and Aging, 22(2), 386-391.

Li, T., Li, J., Qin, H., Guo, X., Wang, X., \& Liu, Z. (2008). Adult attachment and mental health in Chinese college students. Chinese Mental Health Journal, 22(10), 740-743. 
Lin, H., Probst, J., \& Hsu, Y. (2010). Depression among female psychiatric nurses in southern Taiwan: Main and moderating effects of job stress, coping behaviour and social support. Journal of Clinical Nursing, 19(15-16), 2342-2354.

Lipsicas, C. B., \& Mäkinen, I. H. (2010). Immigration and suicidality in the young. Journal of Psychiatry, 55(5), 274-279.

Locke, J. (1694; 1975). An essay concerning human understanding. In J. Barresi (1998). On Becoming a Person. Retrieved from http://jbarresi.psychology.dal.ca/Papers/beperson.html

Lu, L. (1995). The relationship between subjective well-being and psychosocial variables in Taiwan. Journal of Social Psychology, 135(3), 351-35.

Lu, L. (1995). Life events, social support, and depression among Taiwanese female homemakers. The Journal of Social Psychology, 135(2), 185-190.

Macready, G. (2005). Quantitative Research Methods I. Maryland: Macready.

Mahler, M. S., Pine, F., \& Bergman, A. (1973). The Psychological Birth of the Human Infant, New York: Basic Books.

Mak, M. C. K., Bond, M. H., Simpson, J. A., \& Rholes, W. S. (2010). Adult attachment, perceived support, and depressive symptoms in Chinese and American cultures. Journal of Social \& Clinical Psychology, 29(2), 144-165.

Marchand-Reilly, J. F. (2009). Depressive symptoms in young adults: The role of attachment orientations and romantic relationship conflict. Journal of Adult Development, 16(1), 3138.

Maslow, A. H. (1965). Humanistic science and transcendent experiences. Journal of Humanistic Psychology, 5(2), 219-227. 
Maslow, A. H. (1970). Motivation and Personality (2 ${ }^{\text {nd }}$ Ed.). New York: Harper and Row.

McBride, C., Atkinson, L., Quilty, L. C., \& Bagby, R. M. (2006). Attachment as moderator of treatment outcome in major depression: A randomized control trial of interpersonal psychotherapy versus cognitive behavior therapy. Journal of Consulting and Clinical Psychology, 74(6), 1041-1054.

McLanahan, S., \& Garfinkel, I. (2000). The Fragile Families and Child Wellbeing Study: Questions, designs, and a few preliminary results. (Working paper \#00-07 FF). Unpublished manuscript, Princeton, NJ: Princeton University, Center for Research on Child Wellbeing.

Melby, J. N. \& Conger, R. D. (2001). Iowa Family Interaction Rating Scales: Instrument summary. In P. K. Kerig \& K. M. Lindahl (Eds.), Family observation coding systems: Resources for systematic research (pp. 33057). Hillsdale, NJ: Erlbaum.

Milan, S., Snow, S., \& Belay, S. (2009). Depressive symptoms in mothers and children: Preschool attachment as a moderator of risk. Developmental Psychology, 45(4), 10191033.

Monahan, K., \& Lee, J. M. (2008). Adolescent sexual activity: Links between relational context and depressive symptoms. Journal of Youth and Adolescence, 37(8), 917-927.

Muris, P., Mayer, B., \& Meesters, C. (2000). Self-reported attachment style, anxiety, and depression in children. Social Behavior and Personality, 28(2), 157-162.

Park, Y. S., \& Kim, B. S. K. (2008). Asian and European American cultural values and communication styles among Asian American and European American college students. Cultural Diversity and Ethnic Minority Psychology, 14(1), 47-56. 
Pierce, G. R., Sarason, I. G., \& Sarason, B. R. (1991). General and relationship-based perceptions of social support: Are two constructs better than one? Journal of Personality and Social Psychology, 61, 1028-1039.

Pincus, H. A. \& Pettit, A. R. (2001). The societal costs of chronic major depression. In M. Hersen, S. M. Turner, \& D. C. Beidel (Eds.) Adult Psychopathology and Diagnosis. New Jersey: John Wiley \& Sons.

Pistole, M. C. (1989). Attachment in adult romantic relationships: Style of conflict resolution and relationship satisfaction. Journal of Social and Personal Relationships, 6, 505-5510.

Priest, N., Paradies, Y., Stewart, P., \& Luke, J. (2011). Racism and health among urban Aboriginal young people. BioMed Central Public Health, 11(1), 568-576. DOI: $10.1186 / 1471-2458-11-568$

Proulx, C. M., Buehler, C., \& Helms, H. (2009). Moderators of the link between marital hostility and change in spouses' depressive symptoms. Journal of Family Psychology, 23(4), 540550.

Pruchno, R., Wilson-Genderson, M., \& Cartwright, F. P. (2009). Depressive symptoms and marital satisfaction in the context of chronic disease: A longitudinal dyadic analysis. Journal of Family Psychology, 23(4), 573-584.

Radloff, L. S. (1997). The CES-D scale: A self-report depression scale for research in the general population. Applied Psychological Measurement, 1, 385-401.

Raghavan, C. (2006). A study of values as cross-cultural competencies: A potential source of self-esteem? Psicologia: Teoria e Pratica, 8(2), 51-68.

Ritakallio, M., Luukkaala, T., Marttunen, M., Pelkonen, M., \& Kaltiala-Heino, R. (2010). Comorbidity between depression and antisocial behaviour in middle adolescence: The 
role of perceived social support. Nordic Journal of Psychiatry, 64(3), 164-171. DOI: $10.3109 / 08039480903264911$

Rogers, C. (1951). Client-centered therapy: Its current practice, implications and theory. In Rogers, C. (1953). The good life and the fully functioning person. Retrieved from http://www.panarchy.org/rogers/person.html

Rogers, C. (1953). The good life and the fully functioning person. Retrieved from http://www.panarchy.org/rogers/person.html

Rogers, C. (1964). Toward a science of the person. In T. W. Wann (Ed.), Behaviorism and phenomenology: Contrasting bases for modern psychology (pp. 109-140). Chicago, IL: University of Chicago Press.

Maslow, A. H. (1943). A theory of human motivation. Psychological Review, 50(4), 370-396.

Maslow, A. H. (1948). Some theoretical consequences of basic need-gratification. Journal of Personality, 16(4), 402-416. DOI: 10.1111/1467-6494.ep11573146

Ross, C., \& Mirowsky, J. (1984) Men Who Cry. Social Psychology Quarterly, 47(3),138-146.

Rozzini, R. (1996). Prevalence and predictors of depressive symptoms in a nursing home. International Journal of Geriatric Psychiatry, 11 (7), 629-634.

Sable, P. (2007). Accentuating the positive in adult attachments. Attachment and Human Development, 9(4), 361-374.

Sarason, I. G., Sarason, B. R., \& Shearin, E. N. (1986). Social support as an individual difference variable: Its stability, origins, and relational aspects. Journal of Personality and Social Psychology, 50(4), 845-855. 
Sato, T., \& Samuel, R. (2009). Asian international doctoral students' experiences at two American universities: Assimilation, accommodation, and resistance. Journal of Diversity in Higher Education, 2(3), 136-148.

Sawyer-Radloff, L. S. (1977). The CEDS scale: A self-report depression scale for research in the general population. Applied Psychological Measure, 1, 385-401.

Saxena, D. (2009). Quality of Grandparent-Grandchild Relationship in Asian-Indian Immigrant Families. International Journal of Aging and Human Development, 68(4), 321-337.

Shaver, P. R., \& Mikulincer, M. (2007). Adult attachment strategies and the regulation of emotion. In J. J. Gross (Ed.), Handbook of emotion regulation (pp. 446-465). New York: Guilford Press.

Shaver, P. R., Schachner, D. A., \& Mikulincer, M. (2005). Attachment style, excessive reassurance seeking, relationship processes, and depression. Personality and Social Psychology Bulletin, 31(3), 343-359.

Shemmings, D. (2006). Using adult attachment theory to differentiate adult children's internal working models of later life filial relationships. Journal of Aging Studies, 20(2), 177-191.

Silverman, P., Hecht, L., \& McMillin, J. D. (2000). Modeling life satisfaction among the aged: A comparison of Chinese and Americans. Journal of Cross-Cultural Gerontology, 15, 289305.

Skinner, B. F. (1966). Some responses to the stimulus "Pavlov." Journal of the Experimental Analysis of Behavior, 72, 463-465.

Snyder, D. K. (1997). Manual for the Marital Satisfaction Inventory-Revised. Los Angeles: Western Psychological Services. 
Sodowsky, G. R., \& Plake, B. S. (1992). A study of acculturation differences among international people and suggestions for sensitivity to within-group differences. Journal of Counseling and Development, 71, 53-59.

Song, Y., Huang, Y., Liu, D., Kwan, J. S. H., Zhang, F., Sham, P. C., \& Tang, S. W. (2008). Depression in college: Depressive symptoms and personality factors in Beijing and Hong Kong college freshmen. Comprehensive Psychiatry, 49(5), 496-502.

Spanier, G. B. (1976). Measuring dyadic adjustment: New scales for assessing the quality of marriage and similar dyads. Journal of Marriage and the Family, 38, 15-27.

Stein, M. B., Siefert, C. J., Stewart, R. V., \& Hilsenroth, M. J. (2011). Relationship between the Social Cognition and Object Relations Scale (SCORS) and attachment style in a clinical sample . Clinical Psychology \& Psychotherapy, 18(6), 512-523.

Stern, D. N. (1980). The early development of schemas of self, of other, and of various experiences of "self with other." In D. N. Stern (1985). The Interoesinak World of the Infant. New York: Basic Books.

Stern, D. N. (1985). The Interoesinak World of the Infant. New York: Basic Books.

Sternberg, R. J. (1986). A triangular theory of love. Psychological Review, 93(2), 119-135.

Strahan, B. J. (1995). Predictors of depression: An attachment theoretical approach. Journal of Family Studies, 1(1), 33-47.

Sue, D. W., Bucceri, J., Lin, A. I., Nadal, K. L., \& Torino, G. C. (2009). Racial microaggressions and the Asian American experience. Asian American Journal of Psychology, 5(1), 88101.

Sue, D. W., \& Sue, D. (2003; 2007). Counseling the Culturally Diverse: Theory and Practice (Ed. 4 \& 5). New Jersey: Wiley, John \& Sons, Incorporated. 
Sue, S., \& Zane, N. (2009). The role of culture and cultural techniques in psychotherapy: A critique and reformulation. Asian American Journal of Psychology, S(1), 3-14.

Tasca, G. A., Szadkowski, L., Illing, V., Trinneer, A., Grenon, R., Demidenko, N., Krysanski, V., Balfour, L., \& Bissada, H. (2009). Adult attachment, depression, and eating disorder symptoms: The mediating role of affect regulation strategies. Personality and Individual Differences, 47(6), 662-667. DOI: 10.1016/j.paid.2009.06.006

Taylor, S. E., Welch, W. T., Kim, H. S., \& Sherman, D. K. (2007). Cultural differences in the impact of social support on psychological and biological stress responses. Psychological Science, 18(9), 831-837.

Teng, H., Hsu, C., Shih, S., Lu, M., Pan, J., \& Shen, W. (2005). Screening postpartum depression with the Taiwanese version of the Edinburgh Postnatal Depression Scale. Comprehensive Psychiatry, 46(4), 261-265.

Titsworth, S., Hunt, S. L. \& Allen, M. (2009). Quantitative Research in Communication. California: SAGE Publications.

Tochkov, K., Levine, L., \& Sanaka, A. (2010). Variation in the prediction of cross-cultural adjustment by Asian-Indian students in the United States. College Student Journal, 44(3), 677-689.

Verhofstadt, L. L., Buysse, A., Rosseel, Y., \& Peene, O. J. (2006). Confirming the three-factor structure of the Quality of Relationships Inventory within couples. Psychological Assessment, 18(1), 15-21.

Wang, C. D. (2004). Cultural ideal of secure adult attachment: A comparison of three cultural groups (Doctoral dissertation). Available from Ebscohost database. (2005-99006-257) 
Wang, C. D., \& Mallinckrodt, B. S. (2006). Differences between Taiwanese and U.S. cultural beliefs about ideal adult attachment. Journal of Counseling Psychology, 53(2), 192-204.

Wang, C. D., \& Ratanasiripong, P. (2010). Adult attachment, cultural orientation, and psychosocial functioning of Chinese American college students. Cultural Diversity and Ethnic Minority Psychology, 16(2), 101-109.

Wang, C. D., \& Scalise, D. A. (2010). Adult attachment, culturally adjusted attachment, and interpersonal difficulties of Taiwanese adults. The Counseling Psychologist, 38(1), 6-31.

Wang, P. S., Simon, G. E., Avorn, J., Azocar, F., Ludman, E. J., McCulloch, J., Petukhova, M. Z., \& Kessler, R. C. (2007). Telephone screening, outreach, and care management for depressed workers and impact on clinical and work productivity outcomes. Journal of the American Medical Association, 298(12), 1401-1411.

Ward, C., \& Kennedy, A. (1994). Acculturation strategies, psychological adjustment, and sociocultural competence during cross-cultural transitions. International Journal of Intercultural Relations, 18, 329-343.

Ward, C., \& Kennedy, A. (1999). The measurement of sociocultural adaptation. International Journal of Intercultural Relations, 23, 659-677.

Wei, M., Heppner, P. P., Mallen, M., Ku, T., Liao, K. Y., \& Wu, T. (2007). Acculturative stress, perfectionism, years in United States, and depression among Chinese international students. Journal of Counseling Psychology, 54, 385-394.

Wei, M., Russell, D. W., Mallinckrodt, B., \& Vogel, D. L. (2007). The experiences in Close Relationship Scale (ECR)-Short Form: Reliability, validity, and factor structure. Journal of Personality Assessment, 88, 187-204. 
Welsh, D. P., Grello, C. M., \& Harper, M. S. (2003). When love hurts: Depression and adolescent romantic relationships. In P. Florsheim (Ed.). Adolescent romantic relations and sexual behavior: Theory, research, and practical implications (pp. 185211). Mahwah, New Jersey: Lawrence Erlbaum Associates Publishers.

Wittchen, H. U. (1994). Reliability and validity studies of the WHO-Composite International Diagnostic Interview (CIDI): a critical review. Journal of Psychiatry Research, 28, 57 84.

Wong, M.G. (2006). Chinese Americans. In P.G. Min (Ed.), Asian Americans: Contemporary trends and issues (pp. 110-145). Thousand Oaks, CA: Pine Forge Press.

Yang, H., Soong, W., Kuo, P., Chang, H., \& Chen, W. J. (2004). Using the CES-D in a twophase survey for depressive disorders among nonreferred adolescents in Taipei: A stratum-specific likelihood ratio analysis. Journal of Affective Disorders, 82(3), 419-430.

Yeh, C. J. (2003). Age, acculturation, cultural adjustment, and mental health symptoms of Chinese, Korean, and Japanese immigrant youths. Cultural Diversity and Ethnic Minority Psychology, 9(1), 34-48.

Yeh, C. J., \& Wang, Y. W. (2000). Asian American coping attitudes, sources, and practices: Implications for indigenous counseling strategies. Journal of College Student Development, 41(1), 94-103.

Yen, S., Robins, C. J., \& Lin, N. (2000). A cross-cultural comparison of depressive symptom manifestation: China and the United States. Journal of Consulting and Clinical Psychology, 68(6), 993-999.

Yi, J. K., Lin, G. J., \& Kishimoto, Y. (2003). Utilization of counseling services by international students. Journal of Instructional Psychology, 30, 333-342. 
Zung, W. W. K. (1986). Zung Self-Rating Depression Scale and Depression Status Inventory. In N. Sartorius and T. A. Ban, Assessment of Depression (pp. 221-231). Berlin: Springer. 


\section{APPENDIX A: INVITATION}

Dear Student:

I am a doctoral student at West Virginia University. Under the supervision of my advisor, Dr. Jeffrey Daniels, I am conducting dissertation research to determine the differential effect of attachment styles on the quality of romantic relationships and levels of depressive moods between international students from Taiwan or China and American students. We would like to invite you to participate in this study and to also forward the "invitation to participate" to other students who may also be interested in this study.

In this study, we are asking you to complete a demographic questionnaire, six openended questions, and three measures of quality of romantic relationships, attachment styles, and moods.

The criteria for the American student group are: 1) students currently enrolled in a university or college for undergraduate or graduate level studies in the United States; 2) currently involved in a dating relationship, cohabitation, or marriage; 3) identifying as American (born and raised in the United States, second generation or beyond), and 4) ranging in age from 18 to 40.

The criteria for the Taiwanese or Chinese international student group are: 1) international students from Taiwan or China (self-identifying as Taiwanese or Chinese, born, and raised in Taiwan or China) currently enrolled in a university or college for undergraduate or graduate level studies in the United States; 2) currently involved in a dating relationship, cohabitation, or marriage; and 3) ranging in age from 18 to 40.

If you meet the criteria for either group, please choose this link: https://docs.google.com/spreadsheet/viewform?formkey=dDdkcGtDTGh2MnRMdklpc2psR0Vf c3c6MQ 
You can either click the link or copy or paste it into your Internet browser. It should take approximately 20 to 25 minutes to complete the study. The West Virginia University Institutional Review Board (IRB) has approved this study. After completing the four questionnaires online, you will be asked to provide a valid email address to enter a drawing for one of two \$30 Amazon gift cards. After the data collection is completed, a drawing will be conducted based on the email contact you provide. If you have any questions you may contact the primary investigator, Yi-An Lo, M.A. at yian.luo@gmail.com, or call (240) 421-4791; or the research advisor, Dr. Jeffrey Daniels at Jeffrey.Daniels@mail.wvu.edu, or call at (304) 293-2235. Thank you for your time and assistance.

Sincerely, Yi-An Lo, M.A. Jeffrey Daniels, Ph.D. 


\section{APPENDIX B: FLYER}

Are you a college student from the U.S. or Taiwan/China?

\section{Are you dating/ cohabiting /married?}

Are you between 18 and 40 years old?

Are you interested in the quality of romantic relationships, attachment styles, and moods?

If you answer "Yes" to the four questions above, you are the person I am looking for!

How about completing 4 short questionnaires \& then entering a drawing for one of two \$30

\section{Amazon gift cards?}

Details are described on the attached sheet.

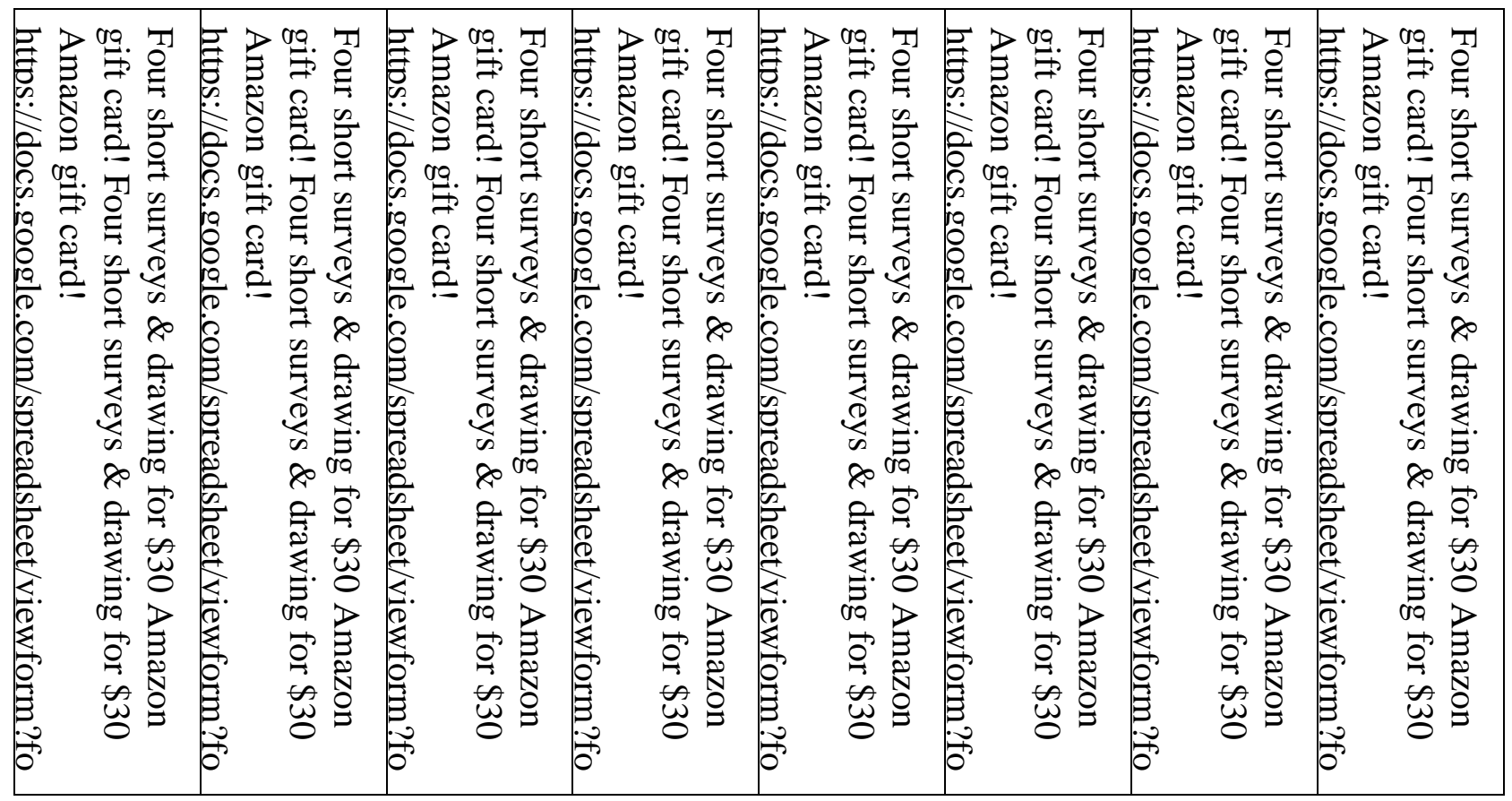




\section{APPENDIX C: INFORMED CONSENT INSTITUTIONAL REVIEW BOARD CONSENT TO PARTICIPATE IN RESEARCH}

Principal Investigator: Lo, Yi-An, M.A.

Dissertation Advisor: Jeffrey Daniels, Ph.D.

Co-Investigator(s): Not applicable.

Department: Counseling, Rehabilitation Counseling, and Counseling Psychology

Tracking Number: H-23806

Study Title: (Dissertation research) Determination of the differential effect of secure attachment styles on the quality of romantic relationships and levels of depressive moods between international students from Taiwan or China and American students.

\section{Contact Persons}

In the event you experience any discomfort or have any concerns related to this research, you should contact the primary investigator, Yi-An Lo, M.A. at yian.luo@ gmail.com, or call (240) 421-4791; or the research advisor, Dr. Jeffrey Daniels at Jeffrey.Daniels@mail.wvu.edu, or call at (304) 293-2235. If you have urgent needs associated with this research, please contact the counseling center at your university or local mental health providers after normal business hours.

The West Virginia University Institutional Review Board (IRB) has approved this study. For information regarding your rights as a research subject, to discuss problems, concerns, or to obtain information or provide suggestions related to the research, contact the Office of Research Compliance at (304) 293-7073. 


\section{Introduction of this Study}

You have been asked to participate in this research study. The participation criteria, procedures for participation, and incentives will be explained in the following section. This research is being conducted to fulfill the requirements for a doctoral dissertation in counseling psychology in the Department of Counseling, Rehabilitation Counseling, and Counseling Psychology at West Virginia University, under the supervision of Dr. Jeffrey Daniels.

\section{Purposes of the Study}

The purpose of this study is to learn more about effect of secure attachment styles on the quality of romantic relationships and levels of depressive moods between international students from Taiwan or China and American students. A total of approximately 120 participants at 30 randomly selected universities in the U.S. are expected to participate in this study.

\section{Description of Procedures}

This study involves completion of a demographic questionnaire, six open-ended questions, and three measures of quality of romantic relationships, attachment styles, and moods, and will take approximately 20 to 25 minutes for you to complete the questionnaires. You do not have to answer all the questions and you can withdraw from participating at any time during the process of filling out questionnaires. We would like to invite you to participate in this study and to also forward the "invitation to participate" to other students who may also be interested in this study.

The criteria for the American student group are 1) students currently enrolled in a university or college for undergraduate or graduate level studies in the United States; 2) currently involved in a dating relationship, cohabitation, or marriage; 3) identifying as American (born and raised in the United States, second generation or beyond), and 4) ranging in age from 18 to 40. 
The criteria for the Taiwanese or Chinese international student group are: 1) international students from Taiwan or China (self-identifying as Taiwanese or Chinese, born, and raised in Taiwan or China) currently enrolled in a university or college for undergraduate or graduate level studies in the United States; 2) currently involved in a dating relationship, cohabitation, or marriage; and 3) ranging in age from 18 to 40 .

If you meet the criteria for either group, please choose this link: https://docs.google.com/spreadsheet/viewform?formkey=dDdkcGtDTGh2MnRMdklpc2psR0Vf c3c6MQ\#gid=0

You can either click the link or copy and paste it into your Internet browser. It should take approximately 20 to 25 minutes to complete the study. After completing the four questionnaires online, you will be asked to provide a valid email address to enter a drawing for one of two \$30 Amazon gift cards. After the data collection is completed, a drawing will be conducted based on the email contact you provide. If you have any questions you may contact the primary investigator, Yi-An Lo, at yian.luo@gmail.com or call (240) 421-479, or the research advisor Dr. Jeffrey Daniels at Jeffrey.Daniels@mail.wvu.edu or call at (304) 293-2235.

\section{Risks and Discomforts}

There are no known or expected risks from participation in this study, except for mild frustration that may be associated with answering the questions.

\section{Alternatives}

You do not have to participate in this study.

\section{Benefits}

After completing the four questionnaires online, you will be asked to provide a valid email address to enter a drawing for one of two $\$ 30$ Amazon gift cards. After the data collection 
is completed, a drawing will be conducted based on the email contact you provided. The knowledge gained from this study may eventually benefit others.

Financial Considerations

There are no fees for participating in this study.

\section{Confidentiality}

Any information about you that is obtained as a result of your participation in this research will be kept as confidential as legally possible. Your responses will not be connected to your personal account(s) on electronic social networks, such as Facebook, MSN, or Yahoo Groups. In any publications that result from this research, neither your name nor any information from which you might be identified will be published without your consent. You can decide to sign or not to sign this authorization section. However, if you choose not to sign this authorization, you will not be able to take part in the research study. Whatever choice you make about this research study will not have an effect on your access to education, health care, or mental health services.

Persons/Organizations receiving the information: The members and staff of any Institutional Review Board (IRB) that oversees this research study.

- West Virginia University Office of Research Compliance and Office of Sponsored Programs.

The following information will be used: Information from your responses to the demographic questionnaire, six open-ended questions, and three measures of quality of romantic relationships, attachment styles, and moods will be used.

The information is being disclosed for the following reasons:

- Review of your data for the research purposes 
- Publication of study results (without identifying you)

- Other research purposes such as developing a better understanding of attachment styles and cultural differences and its impact on moods or quality of romantic relationships for of future culturally competence counseling for Chinese or Taiwanese international or American college students.

You may cancel this authorization at any time by writing to the Principal Investigator: Yi-An Lo at yian.luo@gmail.com or 1099 Valley View Avenue, Lot\#4, Morgantown WV 26505. If you cancel this authorization, any information that was collected already for this study cannot be withdrawn. Once information is disclosed, according to this authorization, the recipient may retrieve it and then the information may no longer be protected by federal regulations. This authorization will not expire unless you cancel it.

\section{Voluntary Participation}

Participation in this study is voluntary. You are free to withdraw your consent to participate in this study at any time. Refusal to participate or withdrawal will not affect your class standing or grades, or access to mental health care and will involve no penalty to you.

In the event new information becomes available that may affect your willingness to participate in this study, this information will be given to you so that you can make an informed decision about whether or not to continue your participation.

Upon signing this form, you can print a copy by clicking file and choose print on the Internet browser.

I willingly consent to participate in this research. 
The participant has had the opportunity to have questions addressed. The participant willingly agrees to be in the study.

Signature of Investigator or

Printed Name

Date

Time

Co-Investigator 


\section{APPENDIX D: DEMOGRAPHIC QUESTIONNAIRE}

1. Please write down your age

2. What is your sex/gender identity? (please circle): Male Female Gay Lesbian Bisexual Transgender Question

3. In what state are you located?

4. Ethnicity or

Home Country

Taiwan China

Caucasian African Native

American American American

Hispanic American Other

How long have you been in the United States?

years

months

Since birth

Since age

6. If you are an American, what generation are you?

Second generation

Third generation

Fourth generation or beyond

7. What level is your degree program in which you are currently enrolled?

Undergraduate

Master's

Doctorate

Professional Degree (e.g., M.D. or J.D.)

8. How long have you been with your current partner?

years months

9. What status would you consider your relationship (please select)?

Dating \& not committed Dating \& committed Cohabiting Married

10. If you are dating your current partner, approximately how many dates have you had with $\operatorname{him} /$ her? 
Dates

11. To the best of your knowledge, is your girlfriend/boyfriend, partner, or spouse participating in this study?

Yes No

12. How many children do you live with?

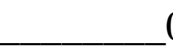

$$
0
$$$$
1
$$
2 3 4 5

13. What is the ethnicity (race/country of origin) of your partner?

Chinese

Asian American, Country of Origin

Taiwanese

Caucasian American

\section{Employment}

Full-Time

Hispanic American

$\underset{\text { American }}{\text { African }} \stackrel{\text { Native }}{\text { American }}$
Other Part-Time Unemployed

Questions:

1) What factors do you think impact relationship quality with this romantic partner?

2) How do these factors (in question one) impact your relationship quality with this romantic partner?

3) How does your cultural background impact your current romantic relationship?

4) How does the American culture impact your current romantic relationship?

5) What are your taboos when communicating with your romantic partner?

6) From your point of view, how do you and your interactions with your partner impact your relationship quality and moods? 


\section{APPENDIX E: QUALITY OF RELATIONSHIPS INVENTORY (QRI; Pierce, Sarason, \& Sarason, 1991).}

Please use the scale below to answer the following questions regarding your relationship with your girlfriend/cohabiting partner/spouse (please circle one).

$\begin{array}{llll}-1- & -2- & -3- & -4- \\ \text { Not at all } & \text { A little } & \text { Quite a bit } & \text { Very much }\end{array}$

1. To what extent could you turn to this person for advice about problems?

$1 \quad 2 \quad 3 \quad 4$

2. How often do you need to work hard to avoid conflict with this person?

$\begin{array}{llll}1 & 2 & 3 & 4\end{array}$

3. To what extent could you count on this person for help with a problem?

$\begin{array}{llll}1 & 2 & 3 & 4\end{array}$

4. How upset does this person sometimes make you feel?

$\begin{array}{llll}1 & 2 & 3 & 4\end{array}$

5. To what extent can you count on this person to give you honest feedback, even if you might not want to hear it?

$\begin{array}{llll}1 & 2 & 3 & 4\end{array}$

6. How much does this person make you feel guilty?

$\begin{array}{llll}1 & 2 & 3 & 4\end{array}$

7. How much do you have to "give in" in this relationship?

$\begin{array}{llll}1 & 2 & 3 & 4\end{array}$

8. To what extent can you count on this person to help you if a family member very close to you died?

$\begin{array}{llll}1 & 2 & 3 & 4\end{array}$


9. How much does this person want you to change?

$1 \quad 2 \quad 3 \quad 4$

10. How positive a role does this person play in your life?

$1 \quad 2 \quad 3 \quad 4$

11. How significant is this relationship in your life?

$\begin{array}{llll}1 & 2 & 3 & 4\end{array}$

12. How close will your relationship be with this person in 10 years?

$\begin{array}{llll}1 & 2 & 3 & 4\end{array}$

13. How much would you miss this person if the two of you could not see or talk with each other for a month?

$1 \quad 2 \quad 3 \quad 4$

14. How critical of you is this person?

$1 \quad 2 \quad 3 \quad 4$

15. If you wanted to go out and do something this evening, how confident are you that this person would be willing to do something with you?

$1 \quad 2 \quad 3 \quad 4$

16. How responsible do you feel for this person's well-being?

$$
1 \quad 2 \quad 3 \quad 4
$$

17. How much do you depend on this person?

$\begin{array}{llll}1 & 2 & 3 & 4\end{array}$

18. To what extent can you count on this person to listen to you when you are very angry at someone else?

$\begin{array}{llll}1 & 2 & 3 & 4\end{array}$


19. How much would you like this person to change?

$\begin{array}{llll}1 & 2 & 3 & 4\end{array}$

20. How angry does this person make you feel?

$1 \quad 2 \quad 3 \quad 4$

21. How much do you argue with this person?

$1 \quad 2 \quad 3 \quad 4$

22. To what extent can you really count on this person to distract you from your worries when you feel under stress?

$1 \quad 2 \quad 3 \quad 4$

23. How often does this person make you feel angry?

$\begin{array}{llll}1 & 2 & 3 & 4\end{array}$

24. How often does this person try to control or influence your life?

$$
1 \quad 2 \quad 3 \quad 4
$$

25. How much more do you give than you get from this relationship?

$\begin{array}{llll}1 & 2 & 3 & 4\end{array}$

Scoring Instructions for the QRI:

The Quality of Relationships Inventory (QRI) yields three scores: (a) social support, (b) depth, and (c) conflict. The Social Support score is computed by averaging the following items:

$1,3,5,8,15,18,22$. The Depth score is computed by averaging the following items: $10,11,12,13$, 16,17. The Conflict score is computed by averaging the following items:

$2,4,6,7,9,14,19,20,21,23,24,25$. 


\section{APPENDIX F: EXPERIENCES IN CLOSE RELATIONSHIP SCALE-SHORT FORM (ECR-S; Wei, Russell, Mallinckrodt, \& Vogel, 2007)}

Instruction: The following statements concern how you feel in romantic relationships. We are interested in how you generally experience relationships, not just in what is happening in a current relationship. Respond to each statement by indicating how much you agree or disagree with it. Mark your answer using the following rating scale:

\begin{tabular}{|c|c|c|c|c|c|c|}
\hline 1 & 2 & 3 & 4 & 5 & 6 & 7 \\
\hline $\begin{array}{c}\text { Strongly } \\
\text { Disagree }\end{array}$ & Disagree & $\begin{array}{c}\text { Slightly } \\
\text { Disagree }\end{array}$ & Neutral & $\begin{array}{c}\text { Slightly } \\
\text { Agree }\end{array}$ & Agree & $\begin{array}{c}\text { Strongly } \\
\text { Agree }\end{array}$ \\
\hline
\end{tabular}

\section{It helps to turn to my romantic partner in times of need.}

2. I need a lot of reassurance that I am loved by my partner.

3. I want to get close to my partner, but I keep pulling back.

4. I find that my partner(s) don't want to get as close as I would like.

\section{I turn to my partner for many things, including comfort and reassurance.}

6. My desire to be very close sometimes scares people away.

7. I try to avoid getting too close to my partner.

\section{I do not often worry about being abandoned.}

\section{I usually discuss my problems and concerns with my partner.}

10. I get frustrated if romantic partners are not available when I need them.

11. I am nervous when partners get too close to me.

12. I worry that romantic partners won't care about me as much as I care about them.

Scoring Information:

Anxiety $=2,4,6,8$ (reverse), 10, 12

Avoidance $=1$ (reverse), 3, 5 (reverse), 7,9 (reverse), 11 


\section{Appendix G: Center for Epidemiologic Studies Depression Scale (CES-D; Radloff, 1977)}

Select the statement that best describes how often you felt this way during the past week.

$\begin{array}{cccc}\begin{array}{c}\text { Rarely or none of the } \\ \text { time }\end{array} & \begin{array}{c}\text { Some or a little of the } \\ \text { time }\end{array} & \begin{array}{c}\text { Occasionally or a } \\ \text { moderate amount of }\end{array} & \begin{array}{c}\text { Most or all of the time } \\ (5-7 \text { days })\end{array} \\ \text { (less than one day) } & (1-2 \text { days }) & \text { time } & (3-4 \text { days })\end{array}$

1 I was bothered by things that usually don't bother me

2 I did not feel like eating; my appetite was poor

3 I felt that I could not shake off the blues even with help from my family and friends

$4 \quad$ I felt that I was just as good as other people

$5 \quad$ I had trouble keeping my mind on what I was doing

$6 \quad$ I felt depressed

7 I felt like everything I did was an effort

8 I felt hopeful about the future

9 I thought my life had been a failure

10 I felt fearful

11 My sleep was restless

12 I was happy

13 I talked less than usual

14 I felt lonely 
15 People were unfriendly

16 I enjoyed life

17 I had crying spells

18 I felt sad

19 I felt that people disliked me

20 I could not 'get going' 